\title{
On the Passage from Atomic to Continuum Theory for Thin Films
}

\author{
BERND SCHMIDT
}

Communicated by K. BHATTACHARYA

\begin{abstract}
We give a rigorous derivation of a continuum theory from atomic models for thin films. This scheme has been proposed by FRIESECKE and JAMES in [J. Mech. Phys. Solids 48, 1519-1540 (2000)]. The resulting continuum energy expression is obtained by integrating a stored energy density which not only depends on the deformation gradient, but also on $v-1$ director fields when $v$ is the (fixed) number of atomic film layers.
\end{abstract}

\section{Contents}

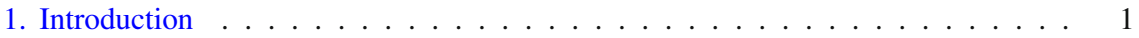

2. Microscopic model and macroscopic variables . . . . . . . . . . . . . 4

2.1. Kinematics . . . . . . . . . . . . . . . . . . 4

2.2. Energy . . . . . . . . . . . . . . . . . . 10

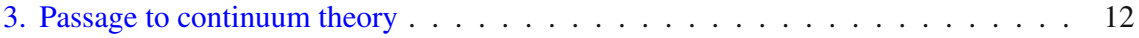

3.1. Main results . . . . . . . . . . . . . . . . . . . . . . 12

3.2. Preparations . . . . . . . . . . . . . . . . . . . . . . 14

3.3. Proof of Theorem $2 \ldots \ldots \ldots \ldots \ldots$

3.4. Proofs of Theorems 1 and $3 \ldots \ldots \ldots \ldots$

3.5. Extension to infinite pair-interactions . . . . . . . . . . . . . . 34

3.6. Extensions and variants . . . . . . . . . . . . . . . . . . . 42

4. Examples/applications . . . . . . . . . . . . . . . . . . . 45

4.1. Pair potentials . . . . . . . . . . . . . . . . . 45

4.2. Pair functionals . . . . . . . . . . . . . . . . . 46

4.3. Angular forces . . . . . . . . . . . . . . . . . . . . 50 50

4.4. A simple example . . . . . . . . . . . . . . . . . . . . . . 51

\section{Introduction}

The main focus of this — and its companion paper [30] — is on the derivation and discussion of effective theories for thin elastic structures. These objects are 
of interest not only in technical applications. One also encounters completely new phenomena (as, for example, large deformations at low energy). To find appropriate energy functionals in the limit of singular geometries is a classical problem in elasticity theory (see, for example, the work of EuLER [15], KIRCHHOFF [24], vON KÁRMÁN [23], etc., also compare [3,11,12,28]). However, rigorous results deriving membrane, plate, rod or shell theories from three-dimensional elasticity have been obtained only recently (see the work of ANZELLOTTI et al. [4], Le Dret and Raoult [25-27] and FRIESECKE et al. [18-22]). By now there has emerged a whole hierarchy of plate theories according to different scalings of the stored energy (compare [20]). For ultra-thin films, that is, films consisting of only few atomic layers, however, a pure continuum mechanical approach might not be justified any more.

Another area of research in elasticity theory concerns the passage from discrete atomic models to continuum theories. Rigorous $\Gamma$-convergence results, especially in one dimension, are proven in [8-10] by BRAIDES and GELLI for pair potentials under suitable growth assumptions on the atomic interactions. A general representation result for bulk energies of distinguishable particles under suitable growth conditions has been obtained by AliCANDRo and CiCALESE [1]. Continuum limits in this regime for thin films are dealt with in a recent paper by ALICANDRo et al. [2]. The results of BLANC et al. [6,7], on the other hand, deal with both pair potential and quantum mechanical energy models, but assume the Cauchy-Born rule to deduce continuum limits in this general framework.

The main goal of this work (see Section 3) is to investigate effective theories of thin films starting from atomistic models in the membrane energy regime. (For recent developments in discrete-to-continuum limits for plates at finite bending energies see [31].) Thus, in order to study new effects that may arise for ultra-thin layers, we consider variational convergence schemes that simultaneously take into account the effects of singular geometries and of atomistic particle interactions. We will prove a rigorous version of a scheme that was proposed by FRIESECKE and JAMES [17]. The resulting continuum energy expression is obtained by integrating a stored energy density which not only depends on the deformation gradient but also on $v-1$ director fields, where $v$ is the (fixed) number of atomic film layers. These vector fields will allow for a fine resolution of the relative layer positions in the small film direction.

More precisely, we fix $h>0$, the thickness of the film, and for $k \in \mathbb{N}$ consider the reference configurations

$$
\mathcal{L}_{k}=\mathbb{Z}^{3} \cap[0, k] \times[0, k] \times[0, h]
$$

(more general lattices are possible, see Paragraph 3.6) subject to some deformation $y^{(k)}: \mathcal{L}_{k} \rightarrow \mathbb{R}^{3}$. The elastic energy of such a deformation is denoted by $E\left(y^{(k)}\right)$. In the membrane energy regime the macroscopic energy scales like the aspect ratio of the film. The natural limiting objects in the limit $k \rightarrow \infty$ are argued to be (after rescaling) given by some function $u:[0,1] \times[0,1] \rightarrow \mathbb{R}^{3}$ (the single layer deformation) and vector fields $b^{i}:[0,1] \times[0,1] \rightarrow \mathbb{R}^{3}, i=1, \ldots, v-1$, where the film consists of $v$ layers of atoms (the relative shifts of the film layers). Having defined a suitable notion of convergence, we are led to the following fundamental 
Problem. Find $\varphi: \mathbb{R}^{3 \cdot 2} \times\left(\mathbb{R}^{3}\right)^{\nu-1} \rightarrow \mathbb{R}$ such that

$$
E\left(u, b^{1}, \ldots, b^{\nu-1}\right):=\lim _{k \rightarrow \infty} \frac{1}{\nu k^{2}} E\left(y^{(k)}\right)=\int_{[0,1]^{2}} \varphi\left(\nabla u, b^{1}, \ldots, b^{\nu-1}\right),
$$

whenever $y^{(k)} \rightarrow\left(u, b^{1}, \ldots, b^{\nu-1}\right)$.

In the spirit of $\Gamma$-convergence (compare, for example, [14]), we do not want to restrict to pointwise limits, but rather calculate a variational limit of the energy that also takes into account microscopic relaxation effects.

In Section 2 we introduce the model. In particular, we discuss the admissible limiting deformations and energy functions that may be considered. We define precisely in what sense microscopic deformations are understood to converge to their macroscopic representatives. Since after suitable interpolation all deformations in our relaxation procedure will have a common Lipschitz constant and a common bound on the relative layer displacements, it is natural to consider the convergence to the single layer deformation $u$ respectively to the relative layer shifts $b^{1}, \ldots, b^{\nu-1}$ in the $w^{*}$-sense in $W^{1, \infty}$ respectively $L^{\infty}$, that is in $\|\cdot\|_{L^{\infty}}$-norm respectively as convergence of localized averages. However, in particular for the latter case, we have to be careful that our interpolation gives the same local averages as the atomic positions only.

The energy of a system of atoms will be supposed to be a frame indifferent function of the atomic positions only required to satisfy mild (and physically reasonable) regularity assumptions. Assumption 2 on the Lipschitz continuity of the energy function implies that small changes in the configuration of the atoms will only result in small changes of their elastic energy, while Assumption 1 on the decay of the interaction energy with respect to atomic distances guarantees that the energy becomes local in the continuum limit.

Section 3 is the core of the theory. It shows how to pass from atomic to continuum theory in the framework set up so far. The scheme follows FRIESECKE and JAMES [17]:

- Replace $u$ and $\mathbf{b}=\left(b^{1}, \ldots, b^{\nu-1}\right)$ by their piecewise affine and piecewise constant approximations $u_{\varepsilon}$ and $\mathbf{b}_{\varepsilon}$, respectively.

- Partition the body into mesoscopic regions where $u_{\varepsilon}, \mathbf{b}_{\varepsilon}$ are affine and constant, respectively, and show that the energy decouples.

- Find minimizers separately on each of these regions.

- Patch them together.

- Obtain an integral expression in terms of $\nabla u$ and $\mathbf{b}$.

We give a rigorous version of these steps which in part were derived formally in [17]. Note, however, that there are some major differences. In particular, the (central) notion of weak neighborhood given here is at variance with that of [17] resulting in some technical differences. These neighborhoods contain those deformations that are close to the limiting objects $u$ and $\mathbf{b}$ over which the energy is minimized. In the limit $k \rightarrow \infty$ we then discover $E(u, \mathbf{b})$ as the limit energy of these relaxed energies. These neighborhoods are thus not only of mathematical interest but also describe physically which deformation fluctuations are subject to relaxation and which will be seen in continuum theory. We will therefore study them in some detail. 
Furthermore, we show that the hypotheses on the decay of the energy and on the regularity of $(u, \mathbf{b})$ made in [17] can be weakened. We also give a proof for the convergence of the relaxed energy on a mesoscale level under homogeneous conditions, thus showing that the continuum theory derived is indeed well-defined. Our study of variants of weak neighborhoods will lead to a representation result for the limiting energy density $\varphi$. The results are extended to systems with unbounded interaction potential. This is of physical interest since many interaction potentials contain terms that diverge for two atoms getting too close to each other. Finally, we discuss some extensions, in particular to certain systems of distinguishable particles and variants of the continuum theory.

In Section 4 we examine physical energy functions and exhibit conditions under which these fit into the theory. In particular, we treat pair potentials, angular forces (to incorporate materials whose binding energy depends on the bond-angles) and pair functionals (derived by the embedded atom method). We show that under reasonable hypotheses on the parameters these energies are admissible for our passage to continuum theory. To give an explicit example we also treat the case of an elementary nearest neighbor model.

It remains to study qualitative aspects of the theory derived here. This will be done in detail in [30]. The dependence of $\varphi$ on the relaxation parameter introduced in Definition 1 measuring the maximal deviations of the atoms from their reference position, which is also connected to the rate of the convergence of the deformations (see Definition 2), will be examined. It turns out that our particular choice of the rate of the convergence is the only rate which allows for atomistic relaxations and gives a non-trivial continuum limit under the decay assumptions on the interaction potential set forth in Assumption 1.

The limiting behavior of $\varphi(A, \mathbf{b})$ under very tensile or compressive strains and convexity properties will be discussed. The results for systems satisfying Assumption 3 turn out to be different from those for nearest neighbor-like interactions as in Paragraph 3.6.2. In [30] we will also consider more realistic mass-spring models for which interesting phenomena will be observed when examining $\varphi$ at $A$ near $O(2,3)$, that is, for deformations that are almost isometric immersions.

\section{Microscopic model and macroscopic variables}

After introducing the atomic model of a thin film subject to some deformation, we identify the variables of continuum theory as limiting points of these deformations. Finally, we collect the basic assumptions on the admissible energy functions.

\subsection{Kinematics}

2.1.1. Atomistic model We consider a film of $v$ atomic layers. Our reference configuration will be

$$
\mathcal{L}_{k}=\mathcal{L} \cap\left(\mathcal{S}_{k} \times[0, h]\right),
$$


where $\mathcal{L}=\mathbb{Z}^{3}, \mathcal{S}_{k}:=[0, k] \times[0, k]$ for $k \in \mathbb{N}$ and $h:=v-1$ is the height of the film. (Only minor changes are necessary to treat more general Bravais-lattices $\mathcal{L}$, see Paragraph 3.6.)

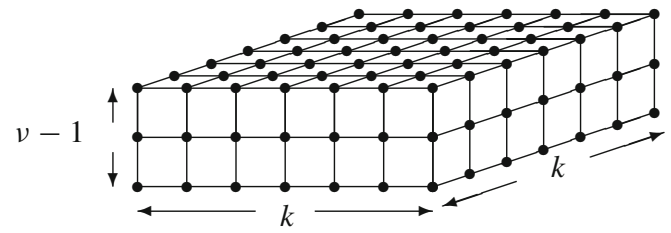

It will sometimes be convenient to enumerate these points as $x_{1}, \ldots, x_{v(k+1)^{2}}$.

The deformations of this configuration will be denoted by

$$
y=y^{(k)}: \mathcal{L}_{k} \rightarrow \mathbb{R}^{3} .
$$

(Also write $y$ as $\left(y_{1}, \ldots, y_{v(k+1)^{2}}\right)$ for $y_{i}=y\left(x_{i}\right)$.) In order for $y$ to be defined not only at the atomic positions, we will assume some interpolation between the atomic positions. However, we then have to be careful that our results do not depend on the particular interpolation chosen, see below.

Our aim being to study the limit $k \rightarrow \infty$, it is natural to introduce the rescaled functions $\tilde{y}$ defined on the common domain $\mathcal{S}_{1} \times[0, h]$ :

$$
\tilde{y}^{(k)}(x):=\frac{1}{k} y^{(k)}\left(k x_{1}, k x_{2}, x_{3}\right) .
$$

Assume for the moment some interpolation is chosen. As pointed out in [17], imposing regularity assumptions on the deformations $y$ implies existence of limiting deformations in the limit $k \rightarrow \infty$. It is argued that these limits have to be considered the natural variables of continuum theory. In detail, the assumptions on the deformations made in [17] are the following. There are constants $c_{1}, c_{2}>0$ such that,

(a) $|y(x)| \leqq c_{2} k$ (boundedness),

(b) $\left|y\left(x_{2}\right)-y\left(x_{1}\right)\right| \leqq c_{2}\left|x_{2}-x_{1}\right|$ (Lipschitz),

(c) $\left|y\left(x_{2}\right)-y\left(x_{1}\right)\right| \geqq c_{1}\left|x_{2}-x_{1}\right|$ (minimal strain hypothesis),

for all $x, x_{1}, x_{2} \in \mathcal{S}_{k} \times[0, h]$.

While conditions (a) and (b) guarantee the existence of well-defined limiting points by weak*-compactness of the set of admissible deformations as $k \rightarrow \infty$, a minimal strain hypothesis is needed in order localize the energy of a deformation. Without that assumption the film could, by repeatedly folding back on itself, be deformed into a block of bulk material. This would certainly not give rise to filmlike behavior.

2.1.2. Macroscopic variables As indicated above, for fixed $c_{2}$ the set of admissible functions $\tilde{y}$ is weak*-compact in $W^{1, \infty}\left(\mathcal{S}_{1} \times[0, h] ; \mathbb{R}^{3}\right)$. Also, $\left(k \tilde{y}_{, 3}^{(k)}\right)$ is bounded in $L^{\infty}\left(\mathcal{S}_{1} \times[0, h] ; \mathbb{R}^{3}\right)$. So there are limit points of these deformations as $k \rightarrow \infty$. There is a $u$ such that (for a subsequence)

$$
\tilde{y}^{(k)} \stackrel{*}{\rightarrow} u, \quad \nabla \tilde{y}^{(k)} \stackrel{*}{\rightarrow} \nabla u \quad \text { in } L^{\infty} .
$$

It is easy to see that $u$ is independent of $x_{3}$. 
There is also a subsequence such that $\left(k \tilde{y}_{, 3}^{(k)}\right)$ weak*-converges in $L^{\infty}$. However, this cannot become a free variable of our continuum theory since the limit function must be determined by the atomic positions only. We instead follow [17] and consider

$$
\Delta^{i} \tilde{y}^{(k)}\left(x_{p}\right)=\tilde{y}^{(k)}\left(x_{p}, i\right)-\tilde{y}^{(k)}\left(x_{p}, 0\right), \quad i=1, \ldots v-1,
$$

$x_{p}=\left(x_{1}, x_{2}\right)$. These quantities measure the relative shift of the layers of the film. By assumption, $\left(k \Delta^{i} \tilde{y}^{(k)}\right)$ is a bounded sequence, and so some subsequence weak*-converges to, say, $b^{i}\left(x_{1}, x_{2}\right)$ :

$$
k\left(\tilde{y}^{(k)}(\cdot, i)-\tilde{y}^{(k)}(\cdot, 0)\right) \stackrel{*}{\rightarrow} b^{i} \quad \text { in } L^{\infty} .
$$

These objects $u$ and $\mathbf{b}=\left(b^{1}, \ldots, b^{v-1}\right)$ constitute the natural variables of a continuum theory.

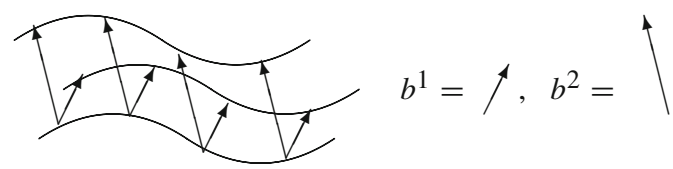

While the first condition (1) does not depend too much on the particular interpolation chosen, we can expect condition (2) to hold only for suitable interpolations (compare below).

In our derivation — deviating from [17]—we will take the point of view that we are given $u$ and $\mathbf{b}=\left(b^{1}, \ldots, b^{\nu-1}\right)$ and would like to assign an energy to these variables allowing for atomistic relaxation. Thus reflecting the fact that we are interested in energies of macroscopic film-like configurations, we do not restrict the lattice deformations themselves but rather impose the following conditions on $u$ and $\mathbf{b}$.

Definition 1. Let $c_{0}>0, u \in W^{1, \infty}\left(\mathcal{S}_{1} ; \mathbb{R}^{3}\right)$ and $\mathbf{b} \in L^{\infty}\left(\mathcal{S}_{1} ;\left(\mathbb{R}^{3}\right)^{\nu-1}\right)$. We say that $(u, \mathbf{b})$ is $c_{0}$-admissible (or simply admissible, if $c_{0}>0$ is understood), that is $(u, \mathbf{b}) \in \mathcal{A}$, if there exists $c_{1}>0$ such that

$$
|u(x)-u(z)| \geqq c_{1}|x-z| \quad \forall x, z \in \mathcal{S}_{1}
$$

(minimal strain hypothesis), and there exists $b^{0} \in L^{\infty}$ such that

$$
\left\|b^{0}\right\|_{L^{\infty}},\left\|b^{i}-b^{0}\right\|_{L^{\infty}} \leqq c_{0}, \quad i=1, \ldots, v-1 .
$$

The first hypothesis ensures the macroscopic deformation to be film-like. The meaning of the second condition will become clear when we have specified our convergence scheme. To be able to work also in un-rescaled variables, we define $U: \mathcal{S}_{k} \rightarrow \mathbb{R}^{3}$ by

$$
\tilde{U}(x)=\frac{1}{k} U(k x)=u(x) .
$$

The following lemma is elementary but important. In particular, the lower bound in (ii) gives a far field minimal strain hypothesis for deformations close to $u$. 
Lemma 1. Suppose $u$ is admissible and $y: \mathcal{L}_{k} \rightarrow \mathbb{R}^{3}$ some deformation with $\sup _{x \in \mathcal{L}_{k}}\left|y(x)-U\left(x_{p}\right)\right| \leqq c$, where $U$ is as in (5). Then y is Lipschitz. Furthermore, for any (rescaled) Lipschitz interpolation y $: \mathcal{S}_{k} \times[0, h] \rightarrow \mathbb{R}^{3}\left(\tilde{y}: \mathcal{S}_{1} \times[0, h] \rightarrow\right.$ $\mathbb{R}^{3}$ ), there are constants $C_{1}, C_{2}, C_{3}>0$ such that,

(i) $\sup _{x \in \mathcal{S}_{1} \times[0, h]}|\tilde{y}(x)| \leqq C_{2}$ and

(ii) $C_{1}|x-z|-C_{3} \leqq|y(x)-y(z)| \leqq C_{2}|x-z| \forall x, \quad z \in \mathcal{S}_{k} \times[0, h]$.

Proof. Since $u$ is admissible, there are $0<c_{1} \leqq c_{2}$ such that

$$
c_{1}|x-z| \leqq|u(x)-u(z)| \leqq c_{2}|x-z|
$$

for all $x, z \in \mathcal{S}_{1}$. Then (i) is clear for $x \in \frac{1}{k} \mathcal{L}_{k} \cap \mathcal{S}_{1}$ : choose $C_{2} \geqq|u(0)|+\sqrt{2} c_{2}+$ $c / k$. For $x, z \in \mathcal{L}_{k},|y(x)-y(z)|$ on the one hand is greater than or equal to

$$
\left|U\left(x_{p}\right)-U\left(z_{p}\right)\right|-2 c \geqq c_{1}\left|x_{p}-z_{p}\right|-2 c \geqq c_{1}|x-z|-c_{1} h-2 c,
$$

which proves the first inequality of (ii) for $x, z \in \mathcal{L}_{k}$. On the other hand, for $x \neq z \in \mathcal{L}_{k}$ this is less than or equal to

$$
\left|U\left(x_{p}\right)-U\left(z_{p}\right)\right|+2 c \leqq c_{2}\left|x_{p}-z_{p}\right|+2 c \leqq c_{2}|x-z|+2 c \leqq C|x-z|
$$

since $|x-z| \geqq 1$. In particular, $y$ is Lipschitz. Choosing a Lipschitz-interpolation with Lipschitz constant $C_{2}$, we get for all $x \in \mathcal{S}_{k} \times[0, h]$

$$
\left|y(x)-U\left(x_{p}\right)\right| \leqq C_{2}+c+\left|U\left(\bar{x}_{p}\right)-U\left(x_{p}\right)\right| \leqq C^{\prime}+c+c_{2}=: c^{\prime},
$$

where $\bar{x} \in \mathcal{L}_{k}$ is such that $|\bar{x}-x| \leqq 1$. Now repeat the above steps to conclude (i) and the first part of (ii) for $y$ on $\mathcal{S}_{k} \times[0, h]\left(\tilde{y}\right.$ on $\left.\mathcal{S}_{1} \times[0, h]\right)$.

Remarks. (i) The constants $C_{1}, C_{2}, C_{3}$ only depend on $u$ through $c, c_{1}$ and $c_{2}$ and on the Lipschitz constant of the chosen interpolation. Below, this constant will be chosen independently of $k$.

(ii) If $y$ is defined only on a subset of $\mathcal{L}_{k}$ and satisfies $|y-U| \leqq c$ on this set, then clearly the implications of the lemma remain valid on this set.

2.1.3. Interpolation and convergence $W^{*} k^{*}$-convergence for bounded sequences in $L^{\infty}$ is equivalent to the convergence of the averages (for example over all sub-squares $a+[0, \alpha]^{2}$ of the domain, compare [13]). We will, therefore, choose our interpolation carefully such that

$$
f_{Q} \tilde{y}(z, i) \mathrm{d} z \approx \frac{1}{\#\left(\frac{1}{k} \mathcal{L} \cap Q\right)} \sum_{z \in \frac{1}{k} \mathcal{L} \cap Q} \tilde{y}(z, i)
$$

for $Q$ a square in $\mathcal{S}_{1}$. For a deformation $y: \mathcal{L}_{k} \rightarrow \mathbb{R}^{3}$ let $\bar{x}=x+(1 / 2,1 / 2)$ for $x \in\{0, \ldots, k-1\}^{2}$ and set

$$
y(\bar{x}, i)=\frac{1}{4} \sum_{\substack{z \in \mathbb{Z}^{2},|z-\bar{x}|=1 / \sqrt{2}}} y(z, i), \quad i=0, \ldots, v-1 .
$$


Now on each of the four triangles with corners $(\bar{x}, i),(z, i),\left(z^{\prime}, i\right)$, where $z, z^{\prime} \in \mathbb{Z}^{2}$ with $|z-\bar{x}|=1 / \sqrt{2},\left|z-z^{\prime}\right|=1$, interpolate linearly to obtain $y(x, i)$ for $x \in \mathcal{S}_{k}$. Interpolating in between the layers is not so subtle, for definiteness we choose $y$ to be linear on the segments $[(x, i-1),(x, i)]$.

Note that this choice guarantees that

$$
f_{\bar{x}+\left[-\frac{1}{2 k}, \frac{1}{2 k}\right]^{2}} \tilde{y}(z, i) \mathrm{d} z=\frac{1}{4} \sum_{z \in \bar{x}+\left\{-\frac{1}{2 k}, \frac{1}{2 k}\right\}^{2}} \tilde{y}(z, i) .
$$

Now let $D \subset \mathcal{S}_{1}$ be some square of fixed side-length $l$ and consider the measure $\rho$ on $\mathbb{R}^{2}$ defined by $\rho=\sum_{x \in \mathbb{Z}^{2}} \delta_{x / k}$, where $\delta_{z}$ is the Dirac-measure at $z$. Supposing $\left|k \Delta^{i} \tilde{y}^{(k)}\right|$ is bounded uniformly in $k$, we get that

$$
\left|f_{D} k \Delta^{i} \tilde{y}\left(z_{1}, z_{2}\right) \mathrm{d} \rho-f_{D} k \Delta^{i} \tilde{y}\left(z_{1}, z_{2}\right) \mathrm{d} z_{1} \mathrm{~d} z_{2}\right| \leqq C \frac{1}{k l} .
$$

This shows that the limits $b^{i}$ are in fact only depending on atomic positions.

In the sequel, we will assume that $y$ (respectively $\tilde{y}$ ) are interpolated precisely in this manner. As a consequence of the next definition and the previous lemma, all deformations that will be taken into account for atomistic relaxation are Lipschitz with a common Lipschitz constant independent of $k$.

Definition 2. Let $u \in W^{1, \infty}\left(\mathcal{S}_{1} ; \mathbb{R}^{3}\right), \mathbf{b} \in L^{\infty}\left(\mathcal{S}_{1} ; \mathbb{R}^{3}\right)$. Choose $c_{0}>0$, a constant. We say that $y^{(k)} \rightarrow(u, \mathbf{b})$ (with respect to $\left.c_{0}\right)$ if

$$
\left\|\tilde{y}^{(k)}-u\right\| \leqq c_{0} / k \text { and } k \Delta^{i} \tilde{y}^{(k)} \stackrel{*}{\rightarrow} b^{i} \quad \text { in } L^{\infty} .
$$

Here and in the sequel we denote by $\|f\|$, respectively $\|\tilde{f}\|$ in rescaled variables,

$$
\|f\|:=\sup _{x \in \mathcal{L}_{k}}|f(x)|, \quad \text { resp. } \quad\|\tilde{f}\|:=\sup _{x \in \mathcal{L}_{k}}\left|\tilde{f}\left(x_{p} / k, x_{3}\right)\right| .
$$

Indeed, $\left\|\tilde{y}^{(k)}-u\right\| \rightarrow 0$ and $\left\|\nabla \tilde{y}^{(k)}\right\|_{L^{\infty}} \leqq$ const. imply $\tilde{y}^{(k)} \stackrel{*}{\rightarrow} u$ in $W^{1, \infty}$. Also note, if $\left\|\tilde{y}^{(k)}-u\right\| \leqq c_{0} / k$, then in fact $k \Delta^{\bar{i}} \tilde{y}^{(k)}$ is bounded, so we can describe weak*-convergence in $L^{\infty}$ by convergence of suitable averages. In order to shed light on the compatibility assumption made for admissible $\mathbf{b}$, we first prove the following lemma.

Lemma 2. Suppose $\left|\tilde{y}^{(k)}(z, i)-u(z)\right| \leqq c_{0} / k$ for all $z \in \frac{1}{k} \mathbb{Z}^{2} \cap \mathcal{S}_{1}$. Then there exist $w^{(k)} \in L^{\infty}\left(\mathcal{S}_{1} ; \mathbb{R}\right)$ with $\left\|w^{(k)}\right\|_{L^{\infty}} \leqq C$ and $w^{(k)} \rightarrow 0$ pointwise almost everywhere as $k \rightarrow \infty$ such that

$$
\left|\tilde{y}^{(k)}(x)-u\left(x_{p}\right)\right| \leqq \frac{c_{0}+w^{(k)}\left(x_{p}\right)}{k} .
$$

Proof. Since there is a common Lipschitz constant for all deformations and $|\tilde{y}(x, i)-u(x)| \leqq c_{0} / k$ whenever $x \in \frac{1}{k} \mathbb{Z}^{2}$, we immediately get a constant $C>c_{0}$ such that

$$
|\tilde{y}(x, i)-u(x)| \leqq C / k \quad \forall x \in \mathcal{S}_{1} .
$$


Let $x \in \mathcal{S}_{1}$ such that $\nabla u(x)$ exists and define $u^{\prime}(x, z)=u(x)+\nabla u(x)(z-$ $x)$. Choose $z_{0} \in\left(\frac{1}{k} \mathbb{Z}^{2}+(1 / 2,1 / 2)\right) \cap \mathcal{S}_{1}$ such that $\left|x-z_{0}\right|$ is minimal and let $\left\{z \in \frac{1}{k} \mathbb{Z}^{2}:\left|z_{0}-z\right|=1 / \sqrt{2}\right\}=\left\{z_{1}, z_{2}, z_{3}, z_{4}\right\}$. Without loss of generality, suppose $x$ lies in the triangle with corners $z_{0}, z_{1}, z_{2}$. By our interpolation and since $u^{\prime}(x, \cdot)$ is affine,

$$
\begin{aligned}
\left|\tilde{y}\left(z_{0}, i\right)-u^{\prime}\left(x, z_{0}\right)\right| & =\left|\frac{1}{4} \sum_{j=1}^{4} \tilde{y}\left(z_{j}, i\right)-\frac{1}{4} \sum_{j=1}^{4} u^{\prime}\left(x, z_{j}\right)\right| \\
& \leqq \frac{1}{4} \sum_{j=1}^{4}\left|\tilde{y}\left(z_{j}, i\right)-u\left(z_{j}\right)\right|+\left|u\left(z_{j}\right)-u^{\prime}\left(x, z_{j}\right)\right| \\
& \leqq \frac{c_{0}}{k}+\frac{1}{4} \sum_{j=1}^{4}\left|u\left(z_{j}\right)-u^{\prime}\left(x, z_{j}\right)\right| .
\end{aligned}
$$

Also, for $j=1,2,3,4$,

$$
\left|\tilde{y}\left(z_{j}, i\right)-u^{\prime}\left(x, z_{j}\right)\right| \leqq \frac{c_{0}}{k}+\left|u\left(z_{j}\right)-u^{\prime}\left(x, z_{j}\right)\right|
$$

Now since $\tilde{y}(\cdot, i)$ and $u^{\prime}(x, \cdot)$ are affine on the triangle with corners $z_{0}, z_{1}, z_{2}$, we deduce from these inequalities that

$$
\begin{aligned}
|\tilde{y}(x, i)-u(x)| & =\left|\tilde{y}(x, i)-u^{\prime}(x, x)\right| \leqq \max _{j \in\{0,1,2\}}\left|\tilde{y}\left(z_{j}, i\right)-u^{\prime}\left(x, z_{j}\right)\right| \\
& \leqq \frac{c_{0}}{k}+\max _{j \in\{1,2,3,4\}}\left|u\left(z_{j}\right)-u^{\prime}\left(x, z_{j}\right)\right| .
\end{aligned}
$$

Choosing

$$
w(x)=\min \left\{C-c_{0}, k \max _{i \in\{1,2,3,4\}}\left|u\left(z_{j}\right)-u^{\prime}\left(x, z_{j}\right)\right|\right\},
$$

we see by (7) and (8) and our choice of interpolating linearly between the film layers

$$
\left|\tilde{y}\left(x_{p}, x_{3}\right)-u\left(x_{p}\right)\right| \leqq \max _{0 \leqq i \leqq v-1}\left|\tilde{y}\left(x_{p}, i\right)-u\left(x_{p}\right)\right| \leqq \frac{c_{0}}{k}+\frac{w\left(x_{p}\right)}{k}
$$

for almost every $\left(x_{1}, x_{2}\right)$. To finish the proof just observe that $z_{j} \rightarrow x$ as $k \rightarrow \infty$ and $\left|u\left(z_{j}\right)-u^{\prime}\left(x, z_{j}\right)\right|=o\left(\left|x-z_{j}\right|\right)=o(1 / k)$ since $\left|x-z_{j}\right| \leqq \sqrt{2} / k$.

As a consequence we obtain the following lemma.

Lemma 3. Suppose $u \in W^{1, \infty}\left(\mathcal{S}_{1}, \mathbb{R}^{3}\right)$, b $\in L^{\infty}\left(\mathcal{S}_{1} ;\left(\mathbb{R}^{3}\right)^{v-1}\right)$. There exists a sequence of deformations $y^{(k)} \rightarrow(u, \mathbf{b})$ if and only if $(4)$ holds. 
Proof. Assume $y^{(k)} \rightarrow(u, \mathbf{b})$ and consider $f^{(k)}(z)=k u(z)-k \tilde{y}^{(k)}(z, 0)$. By the previous lemma, $f^{(k)}$ is bounded in $L^{\infty}$, so there is a weak*-convergent subsequence $f^{\left(k_{j}\right)} \stackrel{*}{\rightarrow} b^{0}$, say. Now if $\chi \in L^{1}\left(\mathcal{S}_{1}\right)$ with $\|\chi\|_{L^{1}}=1$, then by Lemma 2 ,

$$
\int \chi \cdot b^{0}=\lim _{j \rightarrow \infty} \int \chi \cdot f^{\left(k_{j}\right)} \leqq \lim _{j \rightarrow \infty} \int|\chi| \cdot\left|c_{0}+w^{\left(k_{j}\right)}\right|=c_{0}
$$

by dominated convergence since the $w^{(k)}$ are uniformly bounded and converge to zero pointwise. It follows that $\left\|b^{0}\right\|_{L^{\infty}} \leqq c_{0}$. Now considering $k_{j} \Delta^{i} \tilde{y}^{\left(k_{j}\right)}-f^{\left(k_{j}\right) \stackrel{*}{\rightarrow}}$ $b^{i}-b^{0},\left|k_{j} \Delta^{i} \tilde{y}(z)-f^{\left(k_{j}\right)}(z)\right|=|k \tilde{y}(z, i)-k u(z)| \leqq c_{0}+w^{(k)}(z)$, the same reasoning shows that $\left\|b^{i}-b^{0}\right\|_{L^{\infty}} \leqq c_{0}$.

Conversely, suppose $b^{0}$ satisfying (4) exists. Extend $b^{i}$ boundedly (constantly if $b^{i}$ is constant) outside $\mathcal{S}_{1}$. For $0 \leqq i \leqq v-1$ set

$$
\bar{b}^{i}(x)=f_{x+\left[-\frac{1}{2 k}, \frac{1}{2 k}\right]^{2}} b^{i}(z) \mathrm{d} z
$$

Now consider the function $v$ ( $V$ in un-rescaled variables) defined by (interpolation of)

$$
v\left(x_{1}, x_{2}, i\right)=\left\{\begin{array}{l}
u\left(x_{1}, x_{2}\right)-\frac{1}{k} \bar{b}^{0}\left(x_{1}, x_{2}\right) \text { for } i=0, \\
u\left(x_{1}, x_{2}\right)+\frac{1}{k}\left(\bar{b}^{i}\left(x_{1}, x_{2}\right)-\bar{b}^{0}\left(x_{1}, x_{2}\right)\right) \text { for } 1 \leqq i \leqq v-1,
\end{array}\right.
$$

for $\left(x_{1}, x_{2}\right) \in \frac{1}{k} \mathbb{Z}^{2} \cap \mathcal{S}_{1}$. Clearly, $\|v-u\| \leqq c_{0} / k$ since for $x \in \frac{1}{k} \mathbb{Z}^{2} \cap \mathcal{S}_{1}$,

$$
\left|\bar{b}^{0}(x)\right| \leqq\left\|b^{0}\right\|_{L^{\infty}}, \quad\left|\bar{b}^{i}(x)-\bar{b}^{0}(x)\right| \leqq\left\|b^{i}-b^{0}\right\|_{L^{\infty}} .
$$

Also, for each square $D$ of side-length $0<l \leqq 1, f_{D} k \Delta^{i} \tilde{y}=b^{i}+\mathcal{O}(l / k)$ which implies that $k \Delta^{i} \tilde{y} \stackrel{*}{\rightarrow} b^{i}$.

\subsection{Energy}

The energy of a system of $N$ atoms at positions $y_{1}, \ldots, y_{N} \in \mathbb{R}^{3}$ shall be a function $E:\left(\mathbb{R}^{3}\right)^{N} \rightarrow \mathbb{R}$ only depending on atomic positions. To study $E$ we will endow the configuration space $\left(\mathbb{R}^{3}\right)^{N}$ with the norm

$$
\left\|\left(y_{1}, \ldots, y_{N}\right)\right\|=\sup _{1 \leqq i \leqq N}\left|y_{i}\right|_{2} .
$$

The energy of a deformation $y$ is denoted

$$
E(y)=E\left(y(x): x \in \mathcal{L}_{k}\right) .
$$

More generally, the energy of the subset $y(\mathcal{K}), \mathcal{K} \subset \mathcal{L}_{k}$, (counted with multiplicities) of all the atoms is

$$
E(y(\mathcal{K}))=E(y(x): x \in \mathcal{K}) .
$$

We normalize $E$ so that $E(\emptyset)=0$.

Consider deformations $y: \mathcal{K} \rightarrow \mathbb{R}^{3}$, where $\mathcal{K}=\mathcal{L} \cap(\Omega \times[0, h]), \Omega \subset \mathcal{S}_{k}$. For $U$ with $\tilde{U}=u$ as before we write $\|y-U\|=\max _{x \in \mathcal{K}}\left|y(x)-U\left(x_{p}\right)\right|_{2}$. The main assumption on $E$ is the following — physically reasonable-decay hypothesis. 
Assumption 1. Suppose $u$ is admissible. There exists a function $\psi:[0, \infty) \rightarrow \mathbb{R}$ such that

$$
0 \leqq \psi \leqq M \quad \text { and } \quad \psi(r) \leqq M r^{-q},
$$

where $M, q$ are constants, $M>0, q>3$, such that for disjoint sets $\mathcal{M}$ and $\mathcal{N}$ of atoms we have

$$
|E(\mathcal{M} \cup \mathcal{N})-E(\mathcal{M})-E(\mathcal{N})| \leqq \sum_{v \in \mathcal{M}, w \in \mathcal{N}} \psi(|v-w|)
$$

whenever $\|y-U\| \leqq C$. (The function $\psi$ may depend on $C$ and on $u$ through $c_{1}$ and $c_{2}$ where $c_{1}\left|x_{1}-x_{2}\right| \leqq\left|u\left(x_{1}\right)-u\left(x_{2}\right)\right| \leqq c_{2}\left|x_{1}-x_{2}\right|$.)

The energy functionals $E$ act on different spaces because of the different number of atoms involved. The following assumption guarantees that, locally near admissible $u$ s, we have control of $\frac{\partial}{\partial y_{i}} E\left(y_{1}, \ldots, y_{N}\right)$ uniformly in $k$.

Assumption 2. Let $u$ be admissible. We assume that $E$ is locally Lipschitz, and in any $C$-neighborhood of $U$ we have almost everywhere

$$
\left|\frac{\partial}{\partial y_{i}} E(y)\right| \leqq L,
$$

where $L$ might depend on $C$ and on $U$ through $c_{1}, c_{2}$ but is independent of the number of atoms involved.

Furthermore, we assume $E$ to be frame indifferent and only depending on the atomic positions, that is, $E$ remains unchanged after a renumbering of atoms and rigid motions of the configuration $y(\mathcal{K})$.

So in particular $E(\{y\})$, the (finite) self-energy of a single atom at $y \in \mathbb{R}^{3}$, is the same for all $y \in \mathbb{R}^{3}$.

Remarks. (i) By Assumption 2 we could restrict to injective $y$. This would result in energy errors as small as we wish.

(ii) The last requirement can be weakened to situations where $E$ is merely translation invariant and more than one species of atoms is involved. In the latter case one has to assume some periodicity condition. Also systems of distinguishable particles as arise for example in nearest neighbor models can be treated. We will come back to this in Paragraph 3.6.

(iii) Energy functions $E$ satisfying 1 and 2 will be called admissible in the sequel. Note that the set of admissible $E$ forms a vector space.

(iv) The assumption on the Lipschitz continuity can be rephrased by requiring that $\|\nabla E\|_{l^{\infty}(N)}$ be bounded, that is, there be a universal Lipschitz constant when the state space $\mathbb{R}^{N}$ is equipped with the $l^{1}(N)$-norm rather than with the $l^{\infty}(N)$-norm. Then the Lipschitz constant (for the usual norm) in a $C$-neighborhood of $U$ can be chosen as $L \cdot \# \mathcal{K}$, where $L$ might depend on $C, c_{1}, c_{2}$, but is independent of $\mathcal{K}$.

(v) In Paragraph 3.5 we will see that the boundedness assumptions on $\psi$ and $\partial E / \partial y_{i}$ can be weakened. Then also energies that become infinitely large as the distance between two atoms tends to zero can be considered. 
In Lemma 1 we saw how the condition $\|y-U\| \leqq C$ led to a far field minimal strain hypothesis $|y(x)-y(z)| \geqq C_{1}|x-z|-C_{3}$ (with $C_{1}, C_{3}$ depending on $C$ ). In fact, many interesting systems satisfy the above assumptions in a more restrictive sense (see Section 4):

Assumption 3. Assume that $\psi$ and $L$ of Assumption 1 respectively 2 depend only on $C_{1}$ and $C_{3}$ where $y$ satisfies $|y(x)-y(z)| \geqq C_{1}|x-z|-C_{3}$.

This assumption has far reaching consequences as will be detailed in [30]. For the derivation of continuum theory, we will not make use of this.

\section{Passage to continuum theory}

Having defined the variables $u$ and $b^{1}, \ldots, b^{\nu-1}$ of the continuum theory, our aim is to calculate a limit energy $E(u, \mathbf{b})$ as a variational limit of $E\left(y^{(k)}\right)$ as $y^{(k)}$ tends to $(u, \mathbf{b})$. We will prove that this limit exists and give an integral expression in terms of some macroscopic energy density $\varphi$. Furthermore, we will prove a representation formula for $\varphi$. The results will be extended to other atomic systems, in particular to systems with unbounded (pair-) interaction potential.

\subsection{Main results}

Suppose $E$ satisfies Assumptions 1 and 2, and a relaxation parameter $c_{0}>0$ is chosen. Our main result is the following variational convergence result in the spirit of $\Gamma$-convergence:

Theorem 1. There exists a macroscopic stored energy function $\varphi$ such that,

(i) if $y^{(k)} \rightarrow(u, \mathbf{b}),(u, \mathbf{b})$ admissible, then

$$
\liminf _{k \rightarrow \infty} \frac{1}{v k^{2}} E\left(y^{(k)}\right) \geqq E(u, \mathbf{b}) .
$$

(ii) For all admissible $(u, \mathbf{b})$, there exists a sequence $y^{(k)} \rightarrow(u, \mathbf{b})$ such that

$$
\lim _{k \rightarrow \infty} \frac{1}{v k^{2}} E\left(y^{(k)}\right)=E(u, \mathbf{b}) .
$$

Here, $E(u, \mathbf{b})$ is the macroscopic energy

$$
E(u, \mathbf{b})=\int_{\mathcal{S}_{1}} \varphi\left(\nabla u, b^{1}, \ldots, b^{\nu-1}\right) .
$$

In proving this theorem our strategy will be to first reduce to homogeneous conditions and study the limit for affine $u$ and constant $b^{i}$. Assuming this in (12) leads to defining $\varphi$ by solving a cell problem

$$
\varphi(A, \mathbf{b})=\liminf \frac{1}{\nu k^{2}} E\left(y^{(k)}\right) \quad \text { as } y^{(k)} \rightarrow(A, \mathbf{b})
$$


for matrices $A \in \mathbb{R}^{3 \cdot 2}$ of rank 2 and admissible vectors $b^{i} \in \mathbb{R}^{3}$. However, it turns out that there is a more explicit formula for $\varphi$. Let

$\hat{\mathcal{N}}_{k}^{0,1}(A, \mathbf{b})=\left\{y: \mathcal{L}_{k} \rightarrow \mathbb{R}^{3}:\|y-A\| \leqq c_{0}\right.$ and $\left.\frac{1}{(k+1)^{2}} \sum_{x \in \mathbb{Z}^{2} \cap \mathcal{S}_{k}} \Delta^{i} y(x)=b^{i}\right\}$

Then we have the following representation result:

Theorem 2. The macroscopic energy density $\varphi$ of Theorem 3 (and Formula (13)) is given by

$$
\varphi(A, \mathbf{b})=\lim _{k \rightarrow \infty} \frac{1}{\nu k^{2}} \inf _{y \in \hat{\mathcal{N}}_{k}^{0,1}(A, \mathbf{b})} E(y) .
$$

This limit is uniform on compact subsets of $\mathcal{A}_{\mathrm{hom}}$ and depends continuously on $A, \mathbf{b}$.

Here, $\mathcal{A}_{\text {hom }} \subset \mathbb{R}^{3 \cdot 2} \times\left(\mathbb{R}^{3}\right)^{\nu-1}$, the homogeneous version of $\mathcal{A}$ consisting of admissible matrices $A$ and vectors $\mathbf{b}$, is defined by

$$
\begin{aligned}
\mathcal{A}_{\text {hom }}:= & \left\{\left(A, b^{1}, \ldots, b^{\nu-1}\right): \operatorname{rank}(A)=2,\right. \\
& \left.\exists b^{0} \in \mathbb{R}^{3} \text { s.t. }\left|b^{0}\right|, \max _{1 \leqq i \leqq \nu-1}\left|b^{i}-b^{0}\right| \leqq c_{0}\right\} .
\end{aligned}
$$

Measuring the convergence of $k \Delta^{i} \tilde{y}^{(k)}$ in terms of negative Sobolev norms, we get the following sharper version of Theorem 1. In terms of the weak neighborhoods to be introduced in the next paragraph, we will see that this amounts to arbitrarily prescribing the scale of the convergence of the averages as long as the areas over which to take averages are large compared to atomic dimensions.

Theorem 3. Suppose $l=l(k)$ is such that $l(k) \rightarrow 0$ and $k l(k) \rightarrow \infty$ as $k \rightarrow \infty$. Let

$$
\mathcal{W}_{k}^{l}(u, \mathbf{b}):=\left\{y:\|\tilde{y}-u\| \leqq c_{0} / k,\left\|k \Delta^{i} \tilde{y}-b^{i}\right\|_{W^{-1, \infty}} \leqq l\right\}
$$

where $\|f\|_{W^{-1, \infty}}:=\sup \left\{\int f \cdot \chi: \chi \in W_{0}^{1,1},\|\chi\|_{W_{0}^{1,1}}=\int|\nabla \chi|_{2}=1\right\}$. Then

$$
\lim _{k \rightarrow \infty} \frac{1}{\nu k^{2}} \inf _{y \in \mathcal{W}_{k}^{l}(u, \mathbf{b})} E(y)=\int_{\mathcal{S}_{1}} \varphi(\nabla u(x), \mathbf{b}) \mathrm{d} x .
$$

In Paragraph 3.6.2 we will sketch how to extend these results to certain finite range interaction models for distinguishable particle systems.

For many physically interesting models, the requirement that the splitting function $\psi$ be bounded (compare (11)) is too restrictive. More generally, we should allow for energy contributions tending to infinity when atoms are getting very close. 
Theorem 4. Suppose the energy is of the form

$$
E(y)=\frac{1}{2} \sum_{i \neq j} W\left(\left|y_{i}-y_{j}\right|\right)+E_{0}(y),
$$

where $E_{0}$ satisfies the usual assumptions (see Paragraph 2.2, also interactions as discussed in Paragraph 3.6.2 are allowed for $\left.E_{0}\right)$, but $W(r)$ becomes infinitely large as $r$ tends to zero. For any $r_{0}>0$ we assume that $W$ is Lipschitz on $\left[r_{0}, \infty\right)$ and there exist $M=M\left(r_{0}\right) \in \mathbb{R}$ and $q=q\left(r_{0}\right)>3$ such that for (almost every) $r \geqq r_{0}$

$$
|W(r)| \leqq M r^{-q} \quad \text { and } \quad\left|W^{\prime}(r)\right| \leqq M r^{-q+1} .
$$

Then Theorem 1 extends to energy functions of the form (16) where, as in Theorem $2, \varphi: \mathcal{A}_{\mathrm{hom}} \rightarrow(-\infty, \infty]$ is given by $(15)$ and is continuous as a function with values in $\mathbb{R} \cup\{\infty\}$.

Considering $W^{1, \infty}$-weak ${ }^{*}$-converging sequences $\tilde{y}^{(k)}$, it is natural to measure deviations from $u$ in $L^{\infty}$-norm, respectively $\|\cdot\|$. Our choice

$$
\|\tilde{y}-u\| \leqq l_{1}(k)
$$

with $l_{1}(k):=c_{0} / k$ corresponds to a relaxation regime where the individual atoms are allowed to move in a region comparable to atomic dimensions. As is shown in [30], if Assumption 3 holds, $l_{1}=c_{0} / k$ is in fact the only scale which both accounts for atomistic relaxation and yields a non-trivial continuum theory. Moreover, we cannot relax sending the parameter $c_{0}$ to infinity. This is due to our (physically reasonable) decay assumptions on the energy (compare Assumption 1). The main point is that finite $c_{0}$ prevents fracture from happening. Mathematically this could also be achieved by assuming growth conditions on the inter-atomic forces tending to infinity as the distance between initially close atoms becomes large. But this is physically not realistic. In our approach $c_{0}$ enters as a parameter. By its physical interpretation as an upper bound for the deviation of atoms from their macroscopic limit, however, applicability of the theory should be decidable on physical grounds.

Following the proofs in the next paragraphs, it is possible (but tedious) to give explicit error bounds under suitable regularity assumptions on $\nabla u$ and $\mathbf{b}$ (for example requiring them to be (Hölder-)continuous).

\subsection{Preparations}

We are now going to prove these results. Note that in all that follows, $k$ is understood to be sufficiently large, even if not explicitly stated. The constants that will appear in the energy estimates for deformations near some limiting deformation $u$ will depend on $u$, but only through the constants $c_{1}, c_{2}$ (compare below and Assumptions 1 and 2). 
3.2.1. Splitting lemmas We begin our derivation by proving some preparatory lemmas on deformations being close to some admissible $u$ on a part of $\mathcal{S}_{1}$. So let $\Omega \subset \mathcal{S}_{1}$ (usually some mesoscopic sub-square) and consider deformations $y: k \Omega \times[0, h] \rightarrow \mathbb{R}^{3}$. Throughout this paragraph $u: \Omega \rightarrow \mathbb{R}^{3}$ (U in un-rescaled variables) shall satisfy

$$
c_{1}|x-z| \leqq|u(x)-u(z)| \leqq c_{2}|x-z|
$$

for some $0<c_{1} \leqq c_{2}$ and all $x, z \in \Omega$.

From Assumption 1, the following lemma is easily proven by induction.

Lemma 4. If $\mathcal{M}_{1}, \ldots, \mathcal{M}_{n} \subset y(\mathcal{L} \cap(\Omega \times[0, h]))$ are pairwise disjoint sets of atoms and $\|\tilde{y}-u\| \leqq c / k$, then the following inequality holds:

$$
\left|E\left(\mathcal{M}_{1} \cup \ldots \cup \mathcal{M}_{n}\right)-\sum_{j=1}^{n} E\left(\mathcal{M}_{j}\right)\right| \leqq \sum_{1 \leqq i<j \leqq n} \sum_{\substack{v \in \mathcal{M}_{i}, w \in \mathcal{M}_{j}}} \psi(|v-w|) .
$$

In the sequel, we will use the following statements for lattice sums, the proof of which is elementary.

Lemma 5. Let $d \in \mathbb{N}, q>d$. In addition, suppose $c>0$. Then there is a constant $C$ (depending on $c$ ) such that for $a>0$

$$
\sum_{\substack{x \in \mathbb{Z}^{d+1}, 0 \leqq x \\|x| \geqq a}}|x|^{-q} \leqq C a^{d-q} .
$$

The next lemma quantifies the energy for subsets of atoms. It is important as it allows to control the loss of energy when neglecting a (small) set of atoms of the configuration. In particular we will see that $E(\mathcal{M})=\mathcal{O}(\# \mathcal{M})$. Again we are considering deformations $y: k \Omega \times[0, h] \rightarrow \mathbb{R}^{3}$.

Lemma 6. Let $y$ be a deformation satisfying $|\tilde{y}-u| \leqq c / k$ and $\mathcal{K} \subset \mathcal{L} \cap(k \Omega \times$ $[0, h]$ ). Then there is a constant $C$ (not depending on $\mathcal{K}$ ) such that, if $\mathcal{K}=\mathcal{K}_{1} \cup \mathcal{K}_{2}$ for disjoint $\mathcal{K}_{1}$ and $\mathcal{K}_{2}$, then

$$
\left|E(y(x): x \in \mathcal{K})-E\left(y(x): x \in \mathcal{K}_{1}\right)\right| \leqq C \# \mathcal{K}_{2} .
$$

Proof. From Lemma 4 we deduce that

$$
\left|E(y(\mathcal{K}))-E\left(y\left(\mathcal{K}_{1}\right)\right)-\sum_{z \in \mathcal{K}_{2}} E(\{y(z)\})\right| \leqq \sum_{\substack{x \in \mathcal{K} \\ z \in \mathcal{K}_{2}}} \psi(|y(x)-y(z)|) .
$$

By (remark (ii) after) Lemma 1 there are constants $C_{1}$ and $C_{3}$ such that

$$
C_{1}|x-z|-C_{3} \leqq|y(x)-y(z)| \forall x, z \in \mathcal{S}_{k} \times[0, h] .
$$


Now fix $z_{0} \in \mathcal{K}_{2}, y_{0}=y\left(z_{0}\right)$. We will estimate $\sum_{x \in \mathcal{K}} \psi\left(\left|y(x)-y_{0}\right|\right)$ by splitting it into a short-range and a long-range part. Let $\delta=2 C_{3} / C_{1}$. Since the number of $x \in \mathcal{K}$ such that $\left|z_{0}-x\right| \leqq \delta$ is bounded, we find

$$
\sum_{\left\{x:\left|x-z_{0}\right| \leqq \delta\right\}} \psi\left(\left|y(x)-y_{0}\right|\right) \leqq C M,
$$

$M$ being the global bound on $\psi$.

Now if $\left|x-z_{0}\right|>\delta$, then $\frac{C_{1}}{2}\left|x-z_{0}\right|<\left|y(x)-y_{0}\right|$, and we can estimate

$$
\begin{aligned}
\sum_{\left\{x:\left|x-z_{0}\right|>\delta\right\}} \psi\left(\left|y(x)-y_{0}\right|\right) & \leqq \sum_{\left\{x:\left|x-z_{0}\right|>\delta\right\}} M\left|y(x)-y_{0}\right|^{-q} \\
& \leqq \sum_{\left\{x:\left|x-z_{0}\right|>\delta\right\}} M\left(\frac{C_{1}}{2}\right)^{-q}\left|x-z_{0}\right|^{-q} \\
& \leqq C \sum_{\substack{\left\{x \in \mathcal{L}: x \neq 0, 0 \leqq x_{3} \leqq h\right\}}}|x|^{-q} .
\end{aligned}
$$

Since $q>2$, this last expression is bounded by Lemma 5 (with $a=1$ ).

It follows that

$$
\left|E(y(\mathcal{K}))-E\left(y\left(\mathcal{K}_{1}\right)\right)\right| \leqq\left|\sum_{z \in \mathcal{K}_{2}} E(\{y(z)\})\right|+\sum_{z \in \mathcal{K}_{2}} C \leqq C \# \mathcal{K}_{2}
$$

by frame indifference of the energy.

As an immediate consequence we get

Corollary 1. Let $y, y^{\prime}$ be two deformations satisfying the hypotheses of Lemma 6 and $\mathcal{K} \subset \mathcal{L} \cap(k \Omega \times[0, h])$. Then there is a constant $C$ such that

$$
\left|E(y(x): x \in \mathcal{K})-E\left(y^{\prime}(x): x \in \mathcal{K}\right)\right| \leqq C \#\left\{x \in \mathcal{K}: y(x) \neq y^{\prime}(x)\right\} .
$$

Proof. Apply Lemma 6 with $\mathcal{K}_{2}=\left\{x \in \mathcal{K}: y(x) \neq y^{\prime}(x)\right\}$ to $y$ and $y^{\prime}$.

Suppose $Q=[0, a)^{2}, a \leqq 1$, is partitioned by squares $U_{1}, \ldots, U_{r}$ of sidelength $l$, where $1 / k \leqq l \leqq a$, plus some rest $R$ with $|R|=\mathcal{O}\left(a \cdot l^{\prime}\right), l^{\prime} \ll a$, as in the following picture. (Then $r \sim(a / l)^{2}$.)

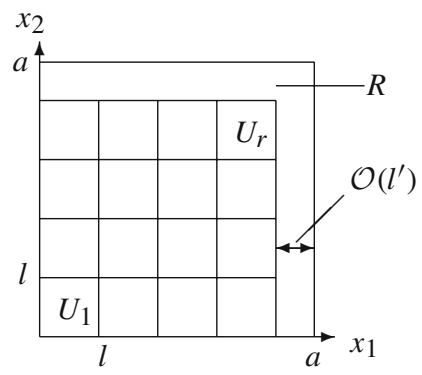


We need to estimate the error when replacing the full energy by the sum of the energies over the individual sets $U_{i}$. Let $\mathcal{K}_{i}=\mathcal{L} \cap\left(k U_{i} \times[0, h]\right), \mathcal{K}=\mathcal{L} \cap(k Q \times$ $[0, h])$.

Lemma 7. Suppose $y: k Q \times[0, h]$ satisfies $|\tilde{y}-u| \leqq c / k$ for some admissible $u$. Then

$$
E(y(x): x \in \mathcal{K})=\sum_{i=1}^{r} E\left(y(x): x \in \mathcal{K}_{i}\right)+\mathcal{O}\left(k a^{2} / l\right)+\mathcal{O}\left(k^{2} a l^{\prime}\right) .
$$

Proof. By Lemma 6 we have

$$
\left|E(y(x): x \in \mathcal{K})-E\left(y(x): x \in \bigcup_{i=1}^{r} \mathcal{K}_{i}\right)\right|=\mathcal{O}\left(k^{2} a l^{\prime}\right) .
$$

Lemma 4 implies that

$$
\left|E\left(y(x): x \in \bigcup_{i=1}^{r} \mathcal{K}_{i}\right)-\sum_{i=1}^{r} E\left(y(x): x \in \mathcal{K}_{i}\right)\right| \leqq \frac{1}{2} \sum_{i \neq j} \sum_{\substack{x \in \mathcal{K}_{i} \\ z \in \mathcal{K}_{j}}} \psi(|y(x)-y(z)|) .
$$

Again we will estimate this error term on the right-hand side by splitting it into a short range term (1) where $|x-z| \leqq \delta$ and a long range term (2) where $|x-z|>\delta$, $\delta:=2 C_{3} / C_{1}$.

1. Short range term: Since $|\psi| \leqq M$, we have

$$
\frac{1}{2} \sum_{i \neq j} \sum_{\substack{x \in \mathcal{K}_{i} \\ z \in \mathcal{K}_{j} \\|x-z| \leqq \delta}} \psi(|y(x)-y(z)|) \leqq \frac{1}{2} \sum_{i \neq j} \sum_{\substack{x \in \mathcal{K}_{i} \\ z \in \mathcal{K}_{j} \\|x-z| \leqq \delta}} M
$$

For fixed $x \in \mathcal{K}_{i}$, the number of $z \in \mathcal{L}$ with $|x-z| \leqq \delta$ is bounded. On the other hand, in order to have at least one $z \in \mathcal{K}_{j}$ with $|x-z| \leqq \delta$ and $i \neq j$, we must have $\operatorname{dist}\left(x_{p}, \partial\left(k U_{i}\right)\right) \leqq \delta$. For fixed $i$, the number of these $x$ is bounded by $C k l$, $C$ constant. This yields

$$
\frac{1}{2} \sum_{i \neq j} \sum_{\substack{x \in \mathcal{K}_{i} \\ z \in \mathcal{K}_{j} \\|x-z| \leqq \delta}} M \leqq \frac{1}{2} \sum_{i} \sum_{\substack{x \in \mathcal{K}_{i} \\ \operatorname{dist}\left(x_{p}, \partial k U_{i}\right) \leqq \delta}} C M \leqq \frac{1}{2} \sum_{i} C k l \leqq C k a^{2} / l .
$$

2. Long range term: As in the proof of Lemma $6,|x-z|>\delta$ implies $|y(x)-y(z)|>$ $\frac{C_{1}}{2}|x-z|$ and thus

$$
\frac{1}{2} \sum_{i \neq j} \sum_{\substack{x \in \mathcal{K}_{i} \\ z \in \mathcal{K}_{j} \\|x-z|>\delta}} \psi(|y(x)-y(z)|) \leqq C \sum_{i \neq j} \sum_{\substack{x \in \mathcal{K}_{i} \\ z \in \mathcal{K}_{j} \\|x-z|>\delta}}|x-z|^{-q}
$$


$C$ some constant. Now for fixed $x \in \mathcal{K}_{i}$ with $\operatorname{dist}\left(x_{p}, \partial\left(k U_{i}\right)\right)=: d(x)=d$ we have by Lemma 5 ( $i$ fixed)

$$
C \sum_{j \neq i} \sum_{\substack{z \in \mathcal{K}_{j} \\|x-z|>\delta}}|x-z|^{-q} \leqq C \sum_{\substack{z \in \mathcal{L}, 0 \leqq z_{3} \leqq h \\|x-z| \geqq \max \{\delta, d\}}}|x-z|^{-q} \leqq C(\max \{\delta, d\})^{2-q} .
$$

So we obtain for $i$ fixed:

$$
\frac{1}{2} \sum_{j \neq i} \sum_{\substack{x \in \mathcal{K}_{i} \\ z \in \mathcal{K}_{j} \\|x-z|>\delta}} \psi(|y(x)-y(z)|) \leqq C \sum_{x \in \mathcal{K}_{i}}(\max \{\delta, d(x)\})^{2-q}
$$

The number of $x$ with $d(x) \leqq \delta$ is bounded by $C k l$. So summing over these $x$ will give a term of order $C \delta^{2-q} k \bar{l}=C k l$ in (18). Now let $x$ be such that $d(x)>\delta$. There exists a unique $m \in \mathbb{N}_{0}$ such that $d \in(\delta+m, \delta+m+1]$. The number of points $x$ corresponding to the same $m$ is bounded by $C v(k l-2(\delta+m)) \leqq C k l$. So $(i$ fixed)

$$
\begin{aligned}
\sum_{\substack{x \in \mathcal{K}_{i} \\
\text { with } d(x)>\delta}} d^{2-q} & \leqq \sum_{m} \sum_{\substack{x \in \mathcal{K}_{i} \text { with } \\
d(x) \in(\delta+m, \delta+m+1]}}(\delta+m)^{2-q} \leqq \sum_{m=0}^{\infty} C k l(\delta+m)^{2-q} \\
& \leqq C k l\left[\delta^{2-q}+\sum_{m \geqq \delta} m^{2-q}\right] \leqq C k l\left[\delta^{2-q}+C \delta^{3-q}\right]
\end{aligned}
$$

by Lemma 5 with $c=0$. Hence this part of the sum is also bounded by $C k l$.

So finally summing over $i$ we get the following upper bound for the long range term:

$$
C r k l \leqq C k a^{2} / l
$$

This is the same bound as for the short range term. We have thus shown that the remaining error term is indeed $\mathcal{O}\left(k a^{2} / l\right)$. Together with (17) this yields the desired estimate.

3.2.2. Weak neighborhoods It is illuminating to describe the deformations that we will take into account for the atomistic energy relaxation more directly by weak neighborhoods about the limit points $u$ and $\mathbf{b}$ in terms of the atomic positions. To do so, we consider mesoscopic local averages. As before, set $\rho=\rho^{(k)}=\sum_{x \in \mathbb{Z}_{-}^{2}} \delta_{x / k}$. Let $Q \subset \mathcal{S}_{1}$ be a sub-square of side-length $l_{4}$, and recall the definition of $\mathbf{b}$ from (9). For admissible $u$, b define:

Definition 3. A deformation $y: \mathcal{L} \cap(k Q \times[0, h]) \rightarrow \mathbb{R}^{3}$ (respectively its interpolation) belongs to the weak neighborhood 
(i) $\mathcal{N}_{k, Q}^{l_{1}, l_{2}, l_{3}}(u, \mathbf{b})$ of $(u, \mathbf{b}), l_{3}<l_{4}$, if

$$
\|\tilde{y}-u\| \leqq l_{1} \quad \text { and } \quad\left|f_{\mathcal{D}} k \Delta^{i} \tilde{y}-\bar{b}^{i} d \rho\right| \leqq l_{2}
$$

for all translates $\mathcal{D}$ of $\left[0, l_{3}\right)^{2}$ with $\mathcal{D} \subset \mathcal{S}_{1}$, or

(ii) $\hat{\mathcal{N}}_{k, Q}^{l_{1}, l_{2}, l_{3}}(u, \mathbf{b})$ of $(u, \mathbf{b}), l_{3}<l_{4}$, if

$$
\|\tilde{y}-u\| \leqq l_{1} \quad \text { and } \quad\left|f_{\mathcal{D}_{j}} k \Delta^{i} \tilde{y}-\bar{b}^{i} d \rho\right| \leqq l_{2}
$$

for all $j=1, \ldots, N$, where $\left\{\mathcal{D}_{j}\right\}$ is a partition of $Q$ into squares $\mathcal{D}_{j}$ of sidelength $l_{3}$ (up to some rest $R$ of measure $|R|=\mathcal{O}\left(l_{3} l_{4}\right)$ ) as in the following picture.

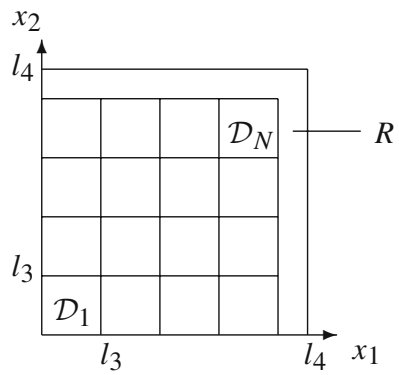

In case $l_{3}=l_{4}$ we require that (19) respectively (20) holds with $\mathcal{D}=Q$ respectively $\mathcal{D}_{1}=Q$.

Remark. Clearly, $\mathcal{N}_{k, Q}^{l_{1}, l_{2}, l_{3}}(u, \mathbf{b}) \subset \hat{\mathcal{N}}_{k, Q}^{l_{1}, l_{2}, l_{3}}(u, \mathbf{b})$, and $V$ as defined in (10) lies in $\mathcal{N}_{k, Q}^{l_{1}, l_{2}, l_{3}}(u, \mathbf{b})$ for admissible $(u, \mathbf{b})$ and $l_{1}=c_{0} / k$. Since we will mainly deal with the choice $l_{1}=c_{0} / k$, we will drop $l_{1}$ from our notation.

Suppose $\Omega \subset \mathcal{S}_{1}$, and for the next lemma assume $\mathbf{b} \in L^{\infty}\left(\Omega ;\left(\mathbb{R}^{3}\right)^{\nu-1}\right)$ satisfies a stronger compatibility condition: there exists $b^{0} \in L^{\infty}\left(\Omega ; \mathbb{R}^{3}\right)$ such that

$$
\left\|b^{0}\right\|_{\infty},\left\|b^{i}-b^{0}\right\|_{\infty} \leqq c_{3}
$$

for all $i \in\{1, \ldots, v-1\}$ and some constant $0<c_{3}<c_{0}$. So $v: \Omega \rightarrow \mathbb{R}^{3}$ as defined in (10) satisfies $\|v-u\| \leqq c_{3}$.

Lemma 8. Suppose $\|y-U\| \leqq c_{0}+\delta, 0 \leqq \delta \leqq c$. Then there exists $y^{\prime}$ with $\left\|y^{\prime}-U\right\| \leqq c_{0}$ such that

$$
\left|f_{D} k \Delta^{i} \tilde{y}^{\prime} \mathrm{d} \rho-f_{D} \bar{b}^{i} \mathrm{~d} \rho\right| \leqq \frac{c_{0}-c_{3}}{c_{0}-c_{3}+\delta}\left|f_{D} k \Delta^{i} \tilde{y} \mathrm{~d} \rho-f_{D} \bar{b}^{i} \mathrm{~d} \rho\right|
$$

whenever $D \subset \Omega, \rho(D)>0$, and $\left|E(y(x): x \in \mathcal{L} \cap(k \Omega \times[0, h]))-E\left(y^{\prime}(x): x \in \mathcal{L} \cap(k \Omega \times[0, h])\right)\right| \leqq C \rho(\Omega) \delta$, where $C=L v \frac{c_{0}+c_{3}}{c_{0}-c_{3}}, L$ as in Assumption 2. 
Proof. Let $v$ be as in (10) and define $y^{\prime}$ such that

$$
\tilde{y}^{\prime}:=\lambda \tilde{y}+(1-\lambda) v, \quad \lambda=\frac{c_{0}-c_{3}}{c_{0}-c_{3}+\delta} .
$$

Then indeed by (21),

$$
\left\|\tilde{y}^{\prime}-u\right\| \leqq \lambda\|\tilde{y}-u\|+(1-\lambda)\|v-u\| \leqq \lambda \frac{c_{0}+\delta}{k}+(1-\lambda) \frac{c_{3}}{k},
$$

whence $\left\|y^{\prime}-U\right\| \leqq c_{0}$. For the local averages observe that

$$
\int_{D} k \Delta^{i} \tilde{y}^{\prime}-\bar{b}^{i} \mathrm{~d} \rho=\lambda \int_{D} k \Delta^{i} \tilde{y}-\bar{b}^{i} \mathrm{~d} \rho .
$$

Now since $\tilde{y}=\frac{1}{\lambda} \tilde{y}^{\prime}-\frac{1-\lambda}{\lambda} v$,

$$
\left\|\tilde{y}-\tilde{y}^{\prime}\right\| \leqq \frac{1-\lambda}{\lambda}\left(\left\|\tilde{y}^{\prime}-u\right\|+\|u-v\|\right) \leqq \frac{\delta}{c_{0}-c_{3}}\left(c_{0} / k+c_{3} / k\right) .
$$

By (remark (iv) after) Assumption 2 the claim follows.

In general, such a uniform bound $c_{3}$ on $\mathbf{b}$ does not exist. So we prove:

Lemma 9. Let $\mathcal{D}_{j}$ be as in Definition 3. Suppose $\left|f_{\mathcal{D}_{j}}\left(k \Delta^{i} \tilde{y}-\bar{b}^{i}\right) \mathrm{d} \rho\right| \leqq \delta \leqq 1$, $j=1, \ldots, N$, and $\|y-U\| \leqq c_{0}+\varepsilon, \varepsilon \leqq 1$. Then there exists $y^{\prime}$ with $\left\|y^{\prime}-U\right\| \leqq c_{0}$,

$$
\left|f_{\mathcal{D}_{j}}\left(k \Delta^{i} \tilde{y}^{\prime}-\bar{b}^{i}\right) \mathrm{d} \rho\right| \leqq \delta, \quad \text { and } \quad\left|E(y)-E\left(y^{\prime}\right)\right| \leqq C\left(\varepsilon^{1 / 5}+\delta^{1 / 4}\right)\left(k l_{4}\right)^{2} .
$$

Proof. We may assume that $\bar{b}^{i}$ is constant on the sets $\mathcal{D}_{j}$ (else for $x \in \mathcal{D}_{j}$ replace $\bar{b}^{i}(x)$ by $f_{\mathcal{D}_{j}} \bar{b}^{i} \mathrm{~d} \rho$ in the sequel). Let $\varepsilon^{\prime}=\varepsilon^{4 / 5}$. First consider those $\mathcal{D}_{j}$ where there do not exist $b^{0}$ and $c_{3} \leqq c_{0}-\varepsilon^{\prime}$ as in the previous lemma. Choose $\bar{b}^{0} \in \mathbb{R}^{3}$ minimizing

$$
\max \left\{\max _{1 \leqq i \leqq \nu-1}\left|\bar{b}^{i}-\bar{b}^{0}\right|,\left|\bar{b}^{0}\right|\right\} \quad\left(\leqq c_{0}\right)
$$

Set

$$
B^{i}=\bar{b}^{i-1}-\bar{b}^{0} \text { for } i=2, \ldots, v, \quad B^{1}=-\bar{b}^{0},
$$

and define $Y^{i}$ and $\overline{Y^{i}}$ by

$$
Y^{i}\left(x_{p}\right)=k\left(\tilde{y}\left(x_{p}, i-1\right)-u\left(x_{p}\right)\right), \quad \overline{Y^{i}}=f_{\mathcal{D}_{j}} Y^{i} \mathrm{~d} \rho
$$

for $i=1, \ldots, v$. Then

$$
\left.\mid \overline{\left(Y^{i}\right.}-\overline{Y^{j}}\right)-\left(B^{i}-B^{j}\right) \mid \leqq 2 \delta \quad \text { for } i, j \in\{1, \ldots, v\} \text {, }
$$


in particular for $a=\overline{Y^{1}}-B^{1}$,

$$
\left|\overline{Y^{i}}-\left(B^{i}+a\right)\right| \leqq 2 \delta .
$$

Since $\left|Y^{i}\right| \leqq c_{0}+\varepsilon$, we also have $\left|\overline{Y^{i}}\right| \leqq c_{0}+\varepsilon$, and it follows that $\left|B^{i}+a\right| \leqq$ $c_{0}+\varepsilon+2 \delta$. By our choice of $\bar{b}^{0}$ there is an $i_{0}$ with $\left|B^{i_{0}}\right| \geqq c_{0}-\varepsilon^{\prime}$ such that $a \cdot B^{i_{0}} \geqq 0$, so $\left|B^{i_{0}}+a\right|^{2} \geqq\left(c_{0}-\varepsilon^{\prime}\right)^{2}+a^{2}$. But then $|a|=\mathcal{O}\left(\sqrt{\varepsilon+\varepsilon^{\prime}+2 \delta}\right)$, that is

$$
\left|\overline{Y^{i}}-B^{i}\right| \leqq C \sqrt{\varepsilon^{\prime}+\delta} \text { for } i=1, \ldots, v \text {. }
$$

Now suppose $i$ is such that $\left|B^{i}\right| \geqq c_{0}-\varepsilon^{\prime}$. To estimate $\left|Y^{i}-B^{i}\right|$, assume without loss of generality that $\overline{Y^{i}}=\left(\overline{Y_{1}^{i}}, 0,0\right), \overline{Y_{1}^{i}} \geqq c_{0}-C \sqrt{\varepsilon^{\prime}+\delta}$. Since $\left|Y^{i}(z)\right| \leqq c_{0}+\varepsilon$ for $z \in \frac{1}{k} \mathbb{Z}^{2} \cap \mathcal{D}_{j}$,

$$
\begin{aligned}
\sum_{z \in \frac{1}{k} \mathbb{Z}^{2} \cap \mathcal{D}_{j}} \mid Y_{1}^{i}(z)-\overline{Y_{1}^{i} \mid} & \leqq \sum_{\substack{z \in \frac{1}{k} \mathbb{Z}^{2} \cap \mathcal{D}_{j} \\
Y_{1}^{i}(z)>\overline{Y_{1}^{i}}}} Y_{1}^{i}(z)-\overline{Y_{1}^{i}}+\sum_{\substack{z \in \frac{1}{k} \mathbb{Z}^{2} \cap \mathcal{D}_{j} \\
Y_{1}^{i}(z) \leqq Y_{1}^{i}}} \overline{Y_{1}^{i}}(z)-Y_{1}^{i} \\
& =2 \sum_{\substack{z \in \frac{1}{k} \mathbb{Z}^{2} \cap \mathcal{D}_{j} \\
Y_{1}^{i}(z)>Y_{1}^{i}}} Y_{1}^{i}(z)-\overline{Y_{1}^{i}}+\sum_{z \in \overline{1}_{k} \mathbb{Z}^{2} \cap \mathcal{D}_{j}} \overline{Y_{1}^{i}}(z)-Y_{1}^{i} \\
& \leqq 2 \sum_{\substack{z \in \frac{1}{k} \mathbb{Z}^{2} \cap \mathcal{D}_{j} \\
Y_{1}^{i}(z)>Y^{i}}} C \sqrt{\varepsilon^{\prime}+\delta}+0 \\
& \leqq C\left(k l_{3}\right)^{2} \sqrt{\varepsilon^{\prime}+\delta} .
\end{aligned}
$$

The second and third component can be estimated by noting that

$$
\left|Y_{m}^{i}(z)\right|^{2} \leqq 2\left(c_{0}+\varepsilon\right)\left(c_{0}+\varepsilon-Y_{1}^{i}(z)\right) \leqq C\left(c_{0}+\varepsilon\right)\left(\left|\overline{Y_{1}^{i}}-Y_{1}^{i}(z)\right|+\sqrt{\varepsilon^{\prime}+\delta}\right)
$$

for $m=2,3$, hence also

$$
\begin{aligned}
\sum_{z \in \frac{1}{k} \mathbb{Z}^{2} \cap \mathcal{D}_{j}}\left|Y_{m}^{i}(z)-\overline{Y_{m}^{i}}\right| \leqq & C \sum_{z \in \frac{1}{k} \mathbb{Z}^{2} \cap \mathcal{D}_{j}} \sqrt{\left|Y_{1}^{i}(z)-\overline{Y_{1}^{i}}\right|}+\sqrt[4]{\varepsilon^{\prime}+\delta} \\
\leqq & C\left(\# \frac{1}{k} \mathbb{Z}^{2} \cap \mathcal{D}_{j}\right)^{1 / 2}\left(\sum_{z \in \frac{1}{k} \mathbb{Z}^{2} \cap \mathcal{D}_{j}}\left|Y_{1}^{i}(z)-\overline{Y_{1}^{i}}\right|\right)^{1 / 2} \\
& +C\left(k l_{3}\right)^{2} \sqrt[4]{\varepsilon^{\prime}+\delta} \\
\leqq & C k l_{3}\left(C\left(k l_{3}\right)^{2} \sqrt{\varepsilon^{\prime}+\delta}\right)^{1 / 2}+C\left(k l_{3}\right)^{2} \sqrt[4]{\varepsilon^{\prime}+\delta} \\
= & C\left(k l_{3}\right)^{2} \sqrt[4]{\varepsilon^{\prime}+\delta}
\end{aligned}
$$


Together with (25) this proves that

$$
\sum_{z \in \frac{1}{k} \mathbb{Z}^{2} \cap \mathcal{D}_{j}}\left|Y^{i}(z)-B^{i}\right| \leqq C\left(k l_{3}\right)^{2}\left(\sqrt[4]{\varepsilon^{\prime}}+\sqrt[4]{\delta}\right) .
$$

Now define a new configuration $y^{\prime \prime}$ by replacing $Y^{i}$ by $B^{i}$ for those $i$ with $\left|B^{i}\right| \geqq c_{0}-\varepsilon^{\prime}$, that is, $Y^{\prime \prime i}$, defined analogously to $Y^{i}$, equals to $B^{i}$ for these $i$ and equals $Y^{i}$ for the other $i$. By (remark (iv) after) Assumption 2,

$$
\left|E\left(y^{\prime \prime}\right)-E(y)\right| \leqq C\left(\sqrt[4]{\varepsilon^{\prime}}+\sqrt[4]{\delta}\right)\left(k l_{4}\right)^{2}
$$

Finally, exactly as in the proof of Lemma 8, we choose $\tilde{y}^{\prime}$ as a convex combination of $\tilde{y}^{\prime \prime}$ and $v$ with $c_{3}=c_{0}-\varepsilon^{\prime}$. Noting that

$$
\left|E\left(y^{\prime}\right)-E\left(y^{\prime \prime}\right)\right| \leqq C \frac{\varepsilon}{\varepsilon^{\prime}}\left(k l_{4}\right)^{2}=C \varepsilon^{1 / 5}\left(k l_{4}\right)^{2}
$$

finishes the proof.

We can now investigate the relationship of the various weak neighborhoods.

Lemma 10. Suppose $u$ and $\mathbf{b}$ are admissible, and scales $0 \leqq l_{2}, l_{2}^{\prime} \leqq 1$ and $1 / k \leqq$ $l_{3}, l_{3}^{\prime} \leqq l_{4}$ are given with $l_{2}^{\prime} \gg l_{3} / l_{3}^{\prime}$.

(i) For all $y \in \hat{\mathcal{N}}_{k, Q}^{l_{2}, l_{3}}(u, \mathbf{b})$ there exists $y^{\prime} \in \hat{\mathcal{N}}_{k, Q}^{0, l_{3}}(u$, b) such that

$$
\left|E\left(y^{\prime}\right)-E(y)\right| \leqq C l_{2}^{1 / 5}\left(k l_{4}\right)^{2} .
$$

If there is $c_{3}<c_{0}$ such that (21) holds, then the error term $\mathcal{O}\left(k^{2} l_{4}^{2} l_{2}^{1 / 5}\right)$ may be replaced by $\mathcal{O}\left(k^{2} l_{4}^{2} l_{2}\right)$.

(ii) $\hat{\mathcal{N}}_{k, Q}^{0, l_{3}}(u, \mathbf{b}) \subset \mathcal{N}_{k, Q}^{l_{2}^{\prime}, l_{3}^{\prime}}(u, \mathbf{b})$.

Proof. Let $y \in \hat{\mathcal{N}}_{k, Q}^{l_{2}, l_{3}}(u, \mathbf{b})$ be arbitrary. Write $Q$ as a disjoint union of $N$ translates of $\left[0, l_{3}\right)^{2}, \mathcal{D}_{1}, \ldots, \mathcal{D}_{N}$, and a rest $R$ whose area is of order $\mathcal{O}\left(l_{3} \cdot l_{4}\right)$ as in Definition 3 (ii). Set $m_{j}^{i}=f_{\mathcal{D}_{j}} k \Delta^{i} \tilde{y}-\bar{b}^{i} \mathrm{~d} \rho$ and define $y_{0}: k Q \times[0, h] \rightarrow \mathbb{R}^{3}$ by (interpolation of)

$$
\tilde{y}_{0}\left(x_{p}, i\right)= \begin{cases}\tilde{y}\left(x_{p}, 0\right) & \text { for } i=0, x_{p} \in \frac{1}{k} \mathcal{L} \cap \mathcal{D}_{j} \\ \tilde{y}\left(x_{p}, i\right)-\frac{1}{k} m_{j}^{i} & \text { for } 1 \leqq i \leqq v-1, x_{p} \in \frac{1}{k} \mathcal{L} \cap \mathcal{D}_{j} \\ \tilde{y}_{0}\left(x_{p}, i\right) & \text { for } 0 \leqq i \leqq v-1, x_{p} \in \frac{1}{k} \mathcal{L} \cap R\end{cases}
$$

Then we have

$$
\left\|y_{0}-y\right\| \leqq \max _{\substack{1 \leqq i \leqq v-1 \\ 1 \leqq j \leqq N}}\left|m_{j}^{i}\right| \leqq l_{2}
$$

since $y \in \hat{\mathcal{N}}_{k, Q}^{l_{2}, l_{3}}(u, \mathbf{b})$. In particular, $\left\|y_{0}-U\right\| \leqq c_{0}+l_{2}$. So because $f_{\mathcal{D}_{j}} k \Delta^{i} \tilde{y}_{0}-$ $\bar{b}^{i} \mathrm{~d} \rho=0$ by construction of $y_{0}$, invoking Lemma 9 (respectively 8), we find $y^{\prime} \in \hat{\mathcal{N}}_{k, Q}^{0, l_{3}}(u, \mathbf{b})$ satisfying

$$
\left|E\left(y^{\prime}\right)-E\left(y_{0}\right)\right| \leqq C l_{2}^{1 / 5}\left(k l_{4}\right)^{2} \quad\left(\text { respectively } \leqq C l_{2}\left(k l_{4}\right)^{2}\right) .
$$


Now by (28) and the Lipschitz Assumption 2 on $E$ we also have

$$
\left|E(y)-E\left(y_{0}\right)\right| \leqq C\left(k l_{4}\right)^{2} l_{2}
$$

This proves (i).

In order to prove (ii), suppose $y \in \hat{\mathcal{N}}_{k, Q}^{0, l_{3}}(u, \mathbf{b})$ and $\mathcal{D} \subset \mathcal{S}_{1}$ is some translate of $\left[0, l_{3}^{\prime}\right)^{2}$. Let $\mathcal{J}$ be the set of those indices of sets $\mathcal{D}_{j}$ that intersect $\mathcal{D}$ and set

$$
\mathcal{D}^{\prime}=\bigcup_{j \in \mathcal{J}} \mathcal{D}_{j}
$$

Then $\rho\left(\left(\mathcal{D}^{\prime} \backslash \mathcal{D}\right) \cup\left(\mathcal{D} \backslash \mathcal{D}^{\prime}\right)\right) \leqq C k^{2} l_{3} l_{3}^{\prime}$, hence since $\left|k \Delta^{i} y-b^{i}\right|$ is bounded,

$$
\begin{aligned}
& \left|\frac{1}{\rho(\mathcal{D})} \int_{\mathcal{D}} k \Delta^{i} y-\bar{b}^{i} \mathrm{~d} \rho-\frac{1}{\rho\left(\mathcal{D}^{\prime}\right)} \int_{\mathcal{D}^{\prime}} k \Delta^{i} y-\bar{b}^{i} \mathrm{~d} \rho\right| \\
& \quad \leqq C \frac{\rho\left(\mathcal{D} \backslash \mathcal{D}^{\prime}\right)}{\rho(\mathcal{D})}+C \frac{\rho\left(\mathcal{D}^{\prime} \backslash \mathcal{D}\right)}{\rho\left(\mathcal{D}^{\prime}\right)}+\left|\left(\frac{1}{\rho(\mathcal{D})}-\frac{1}{\rho\left(\mathcal{D}^{\prime}\right)}\right) \int_{\mathcal{D} \cap \mathcal{D}^{\prime}} k \Delta^{i} y-\bar{b}^{i} \mathrm{~d} \rho\right| \\
& \quad \leqq C \frac{k^{2} l_{3} l_{3}^{\prime}}{\left(k l_{3}^{\prime}\right)^{2}}+C \frac{k^{2} l_{3} l_{3}^{\prime}}{\left(k l_{3}^{\prime}\right)^{2}}+C \frac{k^{2} l_{3} l_{3}^{\prime}}{\left(k l_{3}^{\prime}\right)^{4}}\left(k l_{3}^{\prime}\right)^{2} \\
& \quad=\mathcal{O}\left(l_{3} / l_{3}^{\prime}\right) .
\end{aligned}
$$

But $\int_{\mathcal{D}^{\prime}} k \Delta^{i} y-\bar{b}^{i} \mathrm{~d} \rho=0$, so

$$
\left|f_{\mathcal{D}} k \Delta^{i} y-\bar{b}^{i} \mathrm{~d} \rho\right| \leqq C \frac{l_{3}}{l_{3}^{\prime}} \leqq l_{2}^{\prime},
$$

that is $y \in \mathcal{N}_{k, Q}^{l_{2}^{\prime}, l_{3}^{\prime}}(u, \mathbf{b})$.

The connection between $\mathcal{W}_{k}^{l}(u, \mathbf{b})$ (see Theorem 3 ) and the neighborhoods defined in Definition 3 is described by the following lemma.

Lemma 11. Let $u$, b be admissible. Assume $1 / k \leqq l_{3} \ll l$, and $1 / k \leqq l^{\prime} \ll l_{2}^{\prime} l_{3}^{\prime}$. Then

$$
\hat{\mathcal{N}}_{k}^{0, l_{3}}(u, \mathbf{b}) \subset \mathcal{W}_{k}^{l}(u, \mathbf{b}) \quad \text { and } \quad \mathcal{W}_{k}^{l^{\prime}}(u, \mathbf{b}) \subset \mathcal{N}_{k}^{l_{2}^{\prime}, l_{3}^{\prime}}(u, \mathbf{b}) .
$$

Proof. Suppose $y \in \hat{\mathcal{N}}_{k}^{0, l_{3}}$ and $f \in W_{0}^{1,1}\left(\mathcal{S}_{1} ; \mathbb{R}^{3}\right)$ with $\|f\|_{W_{0}^{1,1}}=1$, without loss of generality $f$ smooth. Choose $x_{j} \in \mathcal{D}_{j}$ such that $\left|\nabla f\left(x_{j}\right)\right| \cdot\left|\mathcal{D}_{j}\right| \leqq \int_{\mathcal{D}_{j}}\left|\nabla f\left(x_{j}\right)\right|$. 
Then

$$
\begin{aligned}
& \int_{\mathcal{S}_{1}} f \cdot\left(k \Delta^{i} \tilde{y}-b^{i}\right)=\frac{1}{k^{2}} \int_{\mathcal{S}_{1}} f \cdot\left(k \Delta^{i} \tilde{y}-\bar{b}^{i}\right) \mathrm{d} \rho+\mathcal{O}(1 / k) \\
& =\frac{1}{k^{2}} \sum_{j} \int_{\mathcal{D}_{j}} f \cdot\left(k \Delta^{i} \tilde{y}-\bar{b}^{i}\right) \mathrm{d} \rho+\mathcal{O}\left(1 / k+l_{3}\right) \\
& \quad=\frac{1}{k^{2}} \sum_{j} \int_{\mathcal{D}_{j}}\left(f\left(x_{j}\right)+\nabla f\left(x_{j}\right)\left(x-x_{j}\right)+o\left(l_{3}\right)\right) \cdot\left(k \Delta^{i} \tilde{y}-\bar{b}^{i}\right) \mathrm{d} \rho+\mathcal{O}\left(l_{3}\right) \\
& \leqq \frac{1}{k^{2}} \sum_{j} \int_{\mathcal{D}_{j}}\left|\nabla f\left(x_{j}\right)\right|\left|x-x_{j}\right| \cdot\left|\left(k \Delta^{i} \tilde{y}-\bar{b}^{i}\right)\right| \mathrm{d} \rho+\mathcal{O}\left(l_{3}\right) \\
& \leqq \frac{1}{k^{2}} \sum_{j} \int_{\mathcal{D}_{j}} C\left|\nabla f\left(x_{j}\right)\right| \sqrt{2} l_{3}+\mathcal{O}\left(l_{3}\right) \\
& \leqq C\left(1+\|\nabla f\|_{L^{1}}\right) l_{3} \leqq C l_{3} \ll l,
\end{aligned}
$$

that is $y \in \mathcal{W}_{k}^{l}(u, \mathbf{b})$. This proves $\hat{\mathcal{N}}_{k}^{0, l_{3}}(u, \mathbf{b}) \subset \mathcal{W}_{k}^{l}(u, \mathbf{b})$.

Now suppose $y \in \mathcal{W}_{k}^{l^{\prime}}(u, \mathbf{b})$ and let $\mathcal{D}$ be some translate of $\left[0, l_{3}^{\prime}\right)^{2} \subset \mathcal{S}_{1}$. Consider the function $f_{a}$ with support in $\mathcal{D}$ and

$$
f_{a}(x)=\frac{1}{4 l_{3}^{\prime}} \min \left\{1, \frac{1}{a} \operatorname{dist}(x, \partial \mathcal{D})\right\} e
$$

for $x \in \mathcal{D}, e \in \mathbb{R}^{3}$ a unit vector. Then for $a \leqq l_{3}^{\prime} / 2$,

$$
\|f\|_{W_{0}^{1,1}}=\|\nabla f\|_{L^{1}}=\frac{1}{4 l_{3}^{\prime} a} \cdot 4\left(l_{3}^{\prime}-a\right) a \leqq 1 .
$$

In particular, sending $a \rightarrow 0$,

$$
\left|\frac{1}{4 l_{3}^{\prime}} \int_{\mathcal{D}} e \cdot\left(k \Delta^{i} \tilde{y}-b^{i}\right)\right|=\lim _{a \rightarrow 0}\left|\int f_{a} \cdot\left(k \Delta^{i} \tilde{y}-b^{i}\right)\right| \leqq l^{\prime} .
$$

This implies

$$
\left|f_{\mathcal{D}}\left(k \Delta^{i} \tilde{y}-\bar{b}^{i}\right) \mathrm{d} \rho\right| \leqq\left|f_{\mathcal{D}}\left(k \Delta^{i} \tilde{y}-b^{i}\right)\right|+\frac{C}{k l_{3}^{\prime}} \leqq \frac{C l^{\prime}}{l_{3}^{\prime}}+\frac{C}{k l_{3}^{\prime}} \ll l_{2}^{\prime},
$$

that is $y \in \mathcal{N}_{k}^{l_{2}^{\prime}, l_{3}^{\prime}}(u, \mathbf{b})$. Therefore, $\mathcal{W}_{k}^{l^{\prime}}(u, \mathbf{b}) \subset \mathcal{N}_{k}^{l_{2}^{\prime}, l_{3}^{\prime}}(u, \mathbf{b})$.

\subsection{Proof of Theorem 2}

In this paragraph we will prove Theorem 2, the representation formula for $\varphi$. Setting

$$
\varphi_{k}(A, \mathbf{b})=\frac{1}{v k^{2}} \inf _{y \in \hat{\mathcal{N}}_{k}(A, \mathbf{b})} E(y)
$$


we need to show that $\varphi_{k}$ converges uniformly on compact subsets of $\mathcal{A}_{\text {hom }}$ to some continuous function $\varphi$. First, we will show that $\varphi$ exists as a pointwise limit (Proposition 1), then in the second part of this paragraph we will investigate the continuity properties of the functions $\varphi_{k}$ (Corollary 2) leading to the final result.

Existence We start with a preparatory lemma. Throughout this paragraph $A \in \mathbb{R}^{3 \cdot 2}$ is some admissible matrix and $\mathbf{b} \in\left(\mathbb{R}^{3}\right)^{\nu-1}$ some admissible vector. Set for short $\hat{\mathcal{N}}_{k}(A, \mathbf{b}):=\hat{\mathcal{N}}_{k, \mathcal{S}_{1}}^{0,1}(A, \mathbf{b})$.

Lemma 12. Suppose $k_{0} \in \mathbb{N}$. Then there is a constant $C$ (independent of $k_{0}$ ) such that, if $k>k_{0}$ is sufficiently large, for every $y \in \hat{\mathcal{N}}_{k_{0}}(A, \mathbf{b})$ there is a $\hat{y} \in \hat{\mathcal{N}}_{k}(A, \mathbf{b})$ with

$$
\left|\frac{1}{\nu k^{2}} E\left(\hat{y}(x): x \in \mathcal{L}_{k}\right)-\frac{1}{\nu k^{2}} E\left(y(x): x \in \mathcal{L}_{k_{0}}\right)\right| \leqq C\left(\frac{1}{k_{0}}+\frac{k_{0}}{k}\right)^{1 / 5} .
$$

Proof. Let $y \in \hat{\mathcal{N}}_{k_{0}}(A, \mathbf{b})$, and cover $\mathcal{S}_{k}$ by translates of $\left[0, k_{0}+1\right)^{2}$, denoted $U_{1}, \ldots, U_{s}$ as in the following picture:

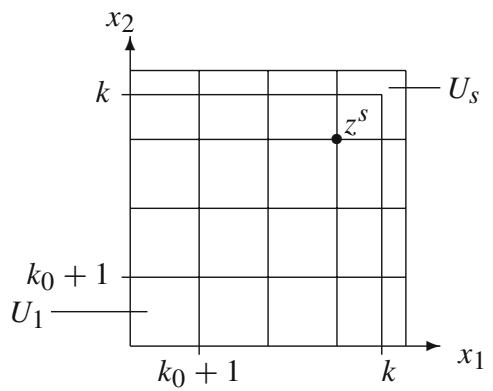

Let $z^{j} \in \mathbb{Z}^{2}$ be the lower left corner of $U_{j}$ and set $f^{j}=A z^{j}$. Then define $y^{\prime}: S \times[0, h] \rightarrow \mathbb{R}^{3}$ by (interpolation of)

$$
y^{\prime}(x):=y\left(x-\left(z_{1}^{j}, z_{2}^{j}, 0\right)\right)+f^{j}
$$

for $x \in \mathcal{L} \cap\left(\left(U_{j} \cap S\right) \times[0, h]\right), 1 \leqq j \leqq s$. It is easy to see that

$$
\left\|y^{\prime}-A\right\| \leqq c_{0} \quad \text { and } \quad\left|f_{\mathcal{S}_{1}} k \Delta^{i} \tilde{y}^{\prime} \mathrm{d} \rho^{(k)}-b^{i}\right|=\mathcal{O}\left(\frac{k_{0}}{k}\right) .
$$

So by Lemma 10 there exists $\hat{y} \in \hat{\mathcal{N}}_{k}(A, \mathbf{b})$ with

$$
\left|\frac{1}{k^{2}} E(\hat{y})-\frac{1}{k^{2}} E\left(y^{\prime}\right)\right| \leqq C\left(\frac{k_{0}}{k}\right)^{1 / 5} .
$$

We estimate the energy of $y^{\prime}$. Using Lemma 7 for translates of $\left[0, \frac{k_{0}+1}{k}\right)^{2}$ and denoting the set of indices $i$ for which $U_{i} \subset \mathcal{S}_{k}$ by $\mathcal{I}$, we see that

$$
\begin{aligned}
E\left(y^{\prime}(x): x \in \mathcal{L}_{k}\right) & =\sum_{i \in \mathcal{I}} E\left(y^{\prime}(x): x \in \mathcal{L} \cap\left(U_{i} \times[0, h]\right)\right)+\mathcal{O}\left(k^{2} / k_{0}+k k_{0}\right) \\
& =\# \mathcal{I} \cdot E\left(y(x): x \in \mathcal{L}_{k_{0}}\right)+\mathcal{O}\left(k^{2} / k_{0}+k_{0} k\right) .
\end{aligned}
$$


by the periodic construction of $y^{\prime}$ and frame indifference. Since \#I $=\left\lfloor k / k_{0}\right\rfloor^{2}=$ $\left(k / k_{0}\right)^{2}\left(1+\mathcal{O}\left(k_{0} / k\right)\right)$, we obtain from (31), noting that $E\left(y(x): x \in \mathcal{L}_{k_{0}}\right)=$ $\mathcal{O}\left(k_{0}^{2}\right)$ by Lemma 6 ,

$$
\frac{1}{\nu k^{2}} E\left(y^{\prime}(x): x \in \mathcal{L}_{k}\right)=\frac{1}{\nu k_{0}^{2}} E\left(y(x): x \in \mathcal{L}_{k_{0}}\right)+\mathcal{O}\left(\frac{1}{k_{0}}\right)+\mathcal{O}\left(\frac{k_{0}}{k}\right) .
$$

This finishes the proof by (30).

Recall the definition of $\varphi_{k}$ from (29).

Proposition 1. The limit

$$
\varphi(A, \mathbf{b}):=\lim _{k \rightarrow \infty} \varphi_{k}(A, \mathbf{b})
$$

exists in $\mathbb{R}$ for all admissible A, b.

Proof. By Lemma 6 we have for $y \in \hat{\mathcal{N}}_{k}(A, \mathbf{b})$

$$
\frac{1}{\nu k^{2}} E\left(y(x): x \in \mathcal{L}_{k}\right)=\mathcal{O}(1)
$$

so $\left(\varphi_{k}(A, \mathbf{b})\right)_{k \in \mathbb{N}}$ is a bounded sequence. We may therefore define $\varphi$ by

$$
\varphi(A, \mathbf{b}):=\liminf _{k \rightarrow \infty} \varphi_{k}(A, \mathbf{b})
$$

For $\delta>0$ we may choose arbitrarily large $k_{0}$ such that $\varphi_{k_{0}}(A, \mathbf{b})<\varphi(A, \mathbf{b})+$ $\delta / 3$. By definition of $\varphi_{k_{0}}$, there also exists $y \in \hat{\mathcal{N}}_{k_{0}}(A, \mathbf{b})$ satisfying $\frac{1}{v k_{0}^{2}} E(y) \leqq$ $\varphi_{k_{0}}(A, \mathbf{b})+\delta / 3$. Now let $k>k_{0}$ be so large that

$$
C\left(\frac{1}{k_{0}}+\frac{k_{0}}{k}\right)^{1 / 5}<\delta / 3
$$

where $C$ is the constant from Lemma 12 . Then there is $\hat{y} \in \hat{\mathcal{N}}_{k}(A, \mathbf{b})$ such that

$$
\begin{aligned}
\frac{1}{v k^{2}} E\left(\hat{y}(x): x \in \mathcal{L}_{k}\right) & \leqq \frac{1}{\nu k_{0}^{2}} E\left(y(x): x \in \mathcal{L}_{k_{0}}\right)+C\left(\frac{1}{k_{0}}+\frac{k_{0}}{k}\right)^{1 / 5} \\
& <\varphi(A, \mathbf{b})+\delta / 3+\delta / 3+\delta / 3
\end{aligned}
$$

It follows $\varphi_{k}(A, \mathbf{b}) \leqq \frac{1}{\nu k^{2}} E\left(\hat{y}(x): x \in \mathcal{L}_{k}\right) \leqq \varphi(A, \mathbf{b})+\delta$.

Since by definition of $\varphi$ also $\varphi_{k}(A, \mathbf{b}) \geqq \varphi(A, \mathbf{b})-\delta$ for $k$ sufficiently large, the proposition is proven. 
Continuity Here we investigate the remaining parts of Theorem 2, namely if $\varphi_{k} \rightarrow \varphi$ uniformly on compact subsets of $\mathcal{A}_{\text {hom }}$ and if $(A, \mathbf{b}) \mapsto \varphi(A, \mathbf{b})$ is continuous. We start by investigating the continuity properties of $\varphi_{k}$, first with respect to the variables $b^{i}$.

Lemma 13. Let $(A, \mathbf{b}),\left(A, \mathbf{b}^{\prime}\right) \in \mathcal{A}_{\text {hom. Then }}$

$$
\left|\varphi_{k}(A, \mathbf{b})-\varphi_{k}\left(A, \mathbf{b}^{\prime}\right)\right| \leqq C\left(\max _{1 \leqq i \leqq \nu-1}\left|b^{i}-b^{\prime i}\right|\right)^{1 / 5},
$$

$C$ a constant (independent of $k$, and on $A$ only depending through $c_{1}, c_{2}$ if the singular values $s_{1}(A) \leqq s_{2}(A)$ of $A$ lie in $\left.\left[c_{1}, c_{2}\right]\right)$.

Proof. For every $y \in \hat{\mathcal{N}}_{k}(A, \mathbf{b}),\left|f_{\mathcal{S}_{1}} k \Delta^{i} \tilde{y} \mathrm{~d} \rho-b^{\prime i}\right| \leqq\left|b^{i}-b^{\prime i}\right|$, that is, $y \in \hat{\mathcal{N}}_{k, \mathcal{S}_{1}}^{l_{2}, 1}\left(A, \mathbf{b}^{\prime}\right)$ for $l_{2}=\max _{i}\left|b^{i}-b^{\prime i}\right|$ fixed. By Lemma 10 ,

$$
\varphi_{k}\left(A, \mathbf{b}^{\prime}\right)=\frac{1}{\nu k^{2}} \inf _{y^{\prime} \in \hat{\mathcal{N}}_{k}\left(A, \mathbf{b}^{\prime}\right)} E\left(y^{\prime}\right) \leqq \frac{1}{\nu k^{2}} E(y)+C l_{2}^{1 / 5} .
$$

Taking the infimum over $y \in \hat{\mathcal{N}}_{k}(A, \mathbf{b})$, we get

$$
\varphi_{k}\left(A, \mathbf{b}^{\prime}\right) \leqq \varphi_{k}(A, \mathbf{b})+C\left(\max _{1 \leqq i \leqq v-1}\left|b^{i}-b^{\prime i}\right|\right)^{1 / 5}
$$

Now interchanging the roles of $\mathbf{b}$ and $\mathbf{b}^{\prime}$ finishes the proof.

In the next lemma we investigate continuity with respect to $A$.

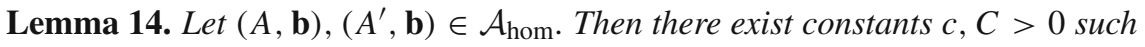
that

$$
\left|\varphi_{k}(A, \mathbf{b})-\varphi_{k}\left(A^{\prime}, \mathbf{b}\right)\right| \leqq k\left|A-A^{\prime}\right|
$$

for $\left|A-A^{\prime}\right|<c / k$.

Proof. Let $y \in \hat{\mathcal{N}}_{k}(A, \mathbf{b})$ and define $y^{\prime}$ by

$$
y^{\prime}(x)=y(x)-A x_{p}+A^{\prime} x_{p} .
$$

Then $\left\|y^{\prime}-y\right\| \leqq\left|A-A^{\prime}\right| \sqrt{2 k^{2}+h^{2}} \leqq C\left|A-A^{\prime}\right| k$, so by Assumption 2,

$$
\left|E\left(y^{\prime}\right)-E(y)\right| \leqq C k^{2}\left|A-A^{\prime}\right| k \text {. }
$$

On the other hand, we clearly have $y^{\prime} \in \hat{\mathcal{N}}_{k}\left(A^{\prime}, \mathbf{b}\right)$. Together with (32) it follows that $\varphi_{k}\left(A^{\prime}, \mathbf{b}\right) \leqq \frac{1}{\nu k^{2}} E(y)+C\left|A-A^{\prime}\right| k$. Since $y$ was arbitrary, we get

$$
\varphi_{k}\left(A^{\prime}, \mathbf{b}\right) \leqq \varphi_{k}(A, \mathbf{b})+C\left|A-A^{\prime}\right| k .
$$

Interchanging the roles of $A$ and $A^{\prime}$ finishes the proof. 
This lemma proves continuity of the $\varphi_{k}$ with respect to $A$. The condition that $\left|A-A^{\prime}\right| \leqq c / k$ can easily be dropped considering intermediate points between $A$ and $A^{\prime}$. However, the Lipschitz constant $C k$ obtained this way blows up as $k \rightarrow \infty$. In order to prove the main continuity result, we therefore need another preparatory lemma:

Lemma 15. Let $(A, \mathbf{b}),\left(A^{\prime}, \mathbf{b}\right) \in \mathcal{A}_{\text {hom }}$ and $c>0$ a constant. Suppose $1 / k \leqq$ $l=l(k) \leqq 1$. Then there is a constant $C>0$ such that

$$
\left|\varphi_{k}(A, \mathbf{b})-\varphi_{k}\left(A^{\prime}, \mathbf{b}\right)\right| \leqq C\left(1 / k l+l+k l\left|A-A^{\prime}\right|\right)
$$

whenever $\left|A-A^{\prime}\right| \leqq c / k l$.

Proof. Cover $\mathcal{S}_{1}$ by translates $U_{1}, \ldots, U_{s}$ of $[0, l)^{2}$ with $\left|\bigcup U_{i} \backslash \mathcal{S}_{1}\right|=\mathcal{O}(l)$ as in the following picture:

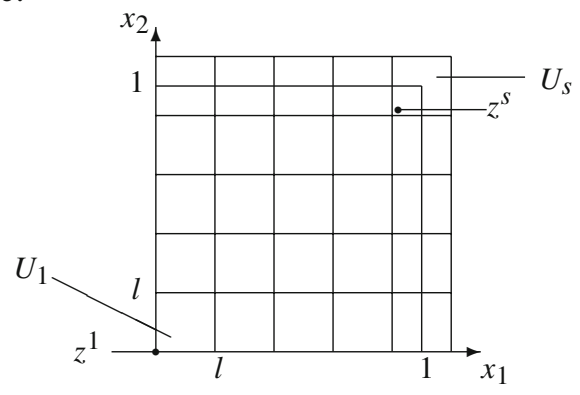

Let $z^{i} \in \mathbb{Z}^{2}$ be the lower left lattice point of $k U_{i}$ and set $f^{i}=\left(A-A^{\prime}\right) z^{i}$. For $y \in \hat{\mathcal{N}}_{k}(A, \mathbf{b})$, we define $y^{\prime}$ by (interpolation and)

$$
y^{\prime}(x)=y(x)-A x_{p}+A^{\prime} x_{p}+f^{i}
$$

if $x \in \mathcal{L} \cap\left(k U_{i} \times[0, h]\right)$. Then

$$
\left\|y^{\prime}-y\right\| \leqq\left|A-A^{\prime}\right| \sqrt{2(k l)^{2}+h^{2}} \leqq C\left|A-A^{\prime}\right| k l \leqq C c,
$$

so Assumption 2 shows that

$$
\left|E\left(y^{\prime}\right)-E(y)\right| \leqq C k^{2} k l\left|A-A^{\prime}\right| .
$$

Now let $\mathcal{I}$ denote the set of those indices $i$ for which $U_{i} \subset \mathcal{S}_{1}$. Applying Lemma 7 to $y^{\prime}$ first, then using frame indifference, and finally applying Lemma 7 to $y^{\prime \prime}(x)=$ $y(x)-A x_{p}+A^{\prime} x_{p}$ gives

$$
\begin{aligned}
E\left(y^{\prime}(x): x \in \mathcal{L}_{k}\right) & =\sum_{i=1}^{r} E\left(y^{\prime}(x): x \in \mathcal{L} \cap\left(k U_{i} \times[0, h]\right)\right)+\mathcal{O}\left(k / l+k^{2} l\right) \\
& =\sum_{i=1}^{r} E\left(y^{\prime \prime}(x): x \in \mathcal{L} \cap\left(k U_{i} \times[0, h]\right)\right)+\mathcal{O}\left(k / l+k^{2} l\right) \\
& =E\left(y^{\prime \prime}(x): x \in \mathcal{L}_{k}\right)+\mathcal{O}\left(k / l+k^{2} l\right) .
\end{aligned}
$$


Since clearly $y^{\prime \prime} \in \hat{\mathcal{N}}_{k}\left(A^{\prime}, \mathbf{b}\right)$, this shows that

$$
\varphi_{k}\left(A^{\prime}, \mathbf{b}\right) \leqq \frac{1}{\nu k^{2}} E\left(y^{\prime \prime}\right) \leqq \frac{1}{\nu k^{2}} E(y)+C\left(1 / k l+l+k l\left|A-A^{\prime}\right|\right)
$$

by (33). Since $y$ was arbitrary, we get

$$
\varphi_{k}\left(A^{\prime}, \mathbf{b}\right) \leqq \varphi_{k}(A, \mathbf{b})+C\left(1 / k l+l+k l\left|A-A^{\prime}\right|\right)
$$

Again interchanging the roles of $A$ and $A^{\prime}$ concludes the proof.

As a consequence of Lemmas 13, 14 and 15 we obtain:

Proposition 2. The set $\left\{\varphi_{k}\right\}$ is equicontinuous.

Proof. Let $\delta>0$ be given. Choose constants $c, C$ as in the previous lemma, and let $l=3 C / k \delta$. Then for $k$ so large that

$$
C l=3 C^{2} / \delta k \leqq \delta / 3
$$

we get from the above lemma for $\left|A-A^{\prime}\right| \leqq c / k l$, that is $\left|A-A^{\prime}\right| \leqq c \delta / 3 C$,

$$
\begin{aligned}
\left|\varphi_{k}(A, \mathbf{b})-\varphi_{k}\left(A^{\prime}, \mathbf{b}\right)\right| & \leqq C\left(1 / k l+l+k l\left|A-A^{\prime}\right|\right) \\
& \leqq \delta / 3+\delta / 3+3 C^{2}\left|A-A^{\prime}\right| / \delta .
\end{aligned}
$$

So for $\left|A-A^{\prime}\right| \leqq \min \left\{\delta^{2} / 9 C^{2}, c \delta / 3 C\right\}$, we have for sufficiently large $k$, say $k>k_{0}$,

$$
\left|\varphi_{k}(A, \mathbf{b})-\varphi_{k}\left(A^{\prime}, \mathbf{b}\right)\right| \leqq \delta
$$

This shows equicontinuity of $\left\{\varphi_{k}(\cdot, \mathbf{b}): k \in \mathbb{N}\right\}$ since the remaining finitely many $\varphi_{1}(\cdot, \mathbf{b}), \ldots, \varphi_{k_{0}}(\cdot, \mathbf{b})$ are continuous by Lemma 14 . By Lemma 13 the family $\left\{\varphi_{k}(A, \cdot): A\right.$ admissible with $\left.s_{1}(A), s_{2}(A) \in\left[c_{1}, c_{2}\right], k \in \mathbb{N}\right\}$ is also equicontinuous for all $c_{2} \geqq c_{1}>0$. The claim follows.

From Propositions 1 and 2 we can now easily finish the proof of Theorem 2.

Proof of Theorem 2. By Proposition $1, \varphi_{k}(A, \mathbf{b}) \rightarrow \varphi(A, \mathbf{b})$ pointwise and, by Proposition $2,\left\{\varphi_{k}\right\}$ is equicontinuous. This implies that $\varphi_{k}(A, \mathbf{b}) \rightarrow \varphi(A, \mathbf{b})$ uniformly on compact subsets of $\mathcal{A}_{\text {hom }}$, in particular that $\varphi$ is continuous since by Arzela-Ascoli every subsequence has a further subsequence that converges. By the pointwise convergence its limit must be $\varphi$. 


\subsection{Proofs of Theorems 1 and 3}

First note that Theorem 1 is an immediate consequence of Theorem 3. So we only have to prove the latter result.

Fix admissible $u \in W^{1, \infty}\left(\mathcal{S}_{1}\right), \mathbf{b} \in L^{\infty}\left(\mathcal{S}_{1}\right)$ and constants $c_{1}, c_{2}>0$ as in (6). We will show that for $l_{3} \rightarrow 0$ and $k l_{3} \rightarrow \infty$,

$$
\lim _{k \rightarrow \infty} \frac{1}{\nu k^{2}} \inf _{\hat{\mathcal{N}}_{k}^{0, l_{3}}(u, \mathbf{b})} E(y)=E(u, \mathbf{b}) .
$$

This will be sufficient since from Lemmas 10 and 11 (and the obvious inclusions of neighborhoods) we obtain the following corollary which precisely describes our relaxation procedure in terms of weak neighborhoods.

Corollary 2. Suppose (34) holds. Then in fact

$$
\lim _{k \rightarrow \infty} \frac{1}{\nu k^{2}} \inf _{y \in \mathcal{U}_{k}(u, \mathbf{b})} E(y)=E(u, \mathbf{b}),
$$

where the minimum is taken over $\mathcal{U}_{k}(u, \mathbf{b})=\hat{\mathcal{N}}_{k}^{l_{2}, l_{3}}(u, \mathbf{b})$ with $l_{2}, l_{3} \rightarrow 0$ and $k l_{3} \rightarrow \infty, \operatorname{or}_{k}(u, \mathbf{b})=\mathcal{W}_{k}^{l}(u, \mathbf{b})$ with $l \rightarrow 0$ and $k l \rightarrow \infty, \operatorname{or} \operatorname{over} \mathcal{U}_{k}(u, \mathbf{b})=$ $\mathcal{N}_{k}^{l_{2}, l_{3}}(u, \mathbf{b})$ with $l_{2}, l_{3} \rightarrow 0$ and $k l_{2} l_{3} \rightarrow \infty$.

If $Q \subset \mathcal{S}_{1}$ is some square in $\mathcal{S}_{1}$ of side-length $l=l(k)$ we write $\hat{\mathcal{N}}_{Q}(u, \mathbf{b}):=$ $\hat{\mathcal{N}}_{k, Q}^{0, l}(u, \mathbf{b})$.

Fix $\sigma>0$ and $0<\delta<\min \left\{1 / 2, c_{1} / 2\right\}$. Since $u \in W^{1, \infty}\left(\mathcal{S}_{1}\right)$, we may choose a measurable set $B \subset \mathcal{S}_{1}$ and $\bar{u} \in C^{1}\left(\mathcal{S}_{1}\right)$ such that $|B| \leqq \sigma$ and

$$
\mathcal{S}_{1} \backslash B=\left\{x \in \mathcal{S}_{1}: u(x)=\bar{u}(x), \nabla u(x)=\nabla \bar{u}(x)\right\} .
$$

Furthermore, there exists $\bar{c}_{2}$ only depending on $c_{2}$ such that $\sup _{x \in \mathcal{S}_{1}}|\nabla \bar{u}(x)| \leqq \bar{c}_{2}$ (compare [16]).

In order to pass from microscopic to macroscopic dimensions, we will introduce a mesoscale $1 / k \ll \varepsilon \leqq l_{3}$. As detailed below, we will consider a partition of $\mathcal{S}_{1}$ by mesoscopic squares $Q_{i}$ of side-length $\varepsilon$ plus some rest $R$ whose area is of the order $\mathcal{O}\left(l_{3}\right)$, see the next picture.

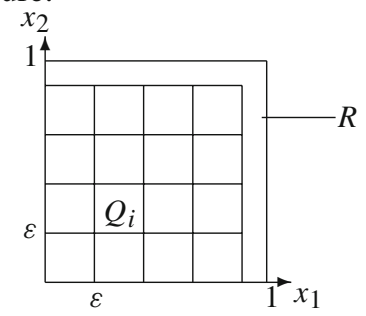

Then $\bar{u} \in C^{1}\left(\mathcal{S}_{1}\right)$ can be approximated by a piecewise affine function $u_{\varepsilon}$. More precisely, there is an increasing and continuous function $g$ only depending on the modulus of continuity of $\nabla \bar{u}$ such that $g(\varepsilon) \rightarrow 0$ as $\varepsilon \rightarrow 0$ and

$$
\left\|\bar{u}-u_{\varepsilon}\right\|_{\infty}<\varepsilon g(\varepsilon),
$$


where $u_{\varepsilon}$ is affine on each of the squares $Q_{i}$. (If $\bar{u} \in C^{1, \alpha}$, one can for example choose $g(\varepsilon)=C \varepsilon^{\alpha}$.) We fix such a function $g$ satisfying (35) from now on.

Let $0<\gamma<1$ be a constant. We choose $\varepsilon^{\prime}=\varepsilon^{\prime}(k)$ such that

$$
k \varepsilon^{\prime} g\left(\varepsilon^{\prime}\right)^{\gamma} \equiv c_{0} .
$$

Note that (35) and (36) imply that

$$
\left\|\bar{u}-u_{\varepsilon}\right\|_{\infty} \ll c_{0} / k \quad \text { if } \varepsilon \leqq \varepsilon^{\prime}
$$

while $\varepsilon^{\prime} \rightarrow 0$ and $k \varepsilon^{\prime} \rightarrow \infty$.

Lemma 16. Let $Q \subset \mathcal{S}_{1}$ be one of the squares $Q_{i}$ (on which $\nabla u_{\varepsilon}$ is constant). Suppose $c_{1}-\delta \leqq s_{1}\left(\nabla u_{\varepsilon}\right) \leqq s_{2}\left(\nabla u_{\varepsilon}\right) \leqq c_{2}+\delta$ on $Q$, and let $\mathbf{b}$ be a constant admissible vector in $\left(\mathbb{R}^{3}\right)^{\nu-1}$. Then if $\varepsilon \leqq \varepsilon^{\prime}$,

$$
\inf _{y \in \hat{\mathcal{N}}_{Q}(u, \mathbf{b})} E(y)-\inf _{y \in \hat{\mathcal{N}}_{Q}\left(u_{\varepsilon}, \mathbf{b}\right)} E(y) \mid \leqq C\left(\delta^{1 / 5}|Q|+\frac{|B \cap Q|}{\delta^{3}}\right) k^{2} .
$$

Proof. Let $y \in \hat{\mathcal{N}}_{Q}(u$, b). We set

$$
r_{Q}:=\#\left\{x \in \frac{1}{k} \mathbb{Z}^{2} \cap Q:|u(x)-\bar{u}(x)|>\delta / k\right\}
$$

and define $y^{\prime}$ by

$$
\tilde{y}^{\prime}(x)= \begin{cases}\tilde{y}(x) & \text { if }\left|u\left(x_{p}\right)-\bar{u}\left(x_{p}\right)\right| \leqq \delta / k \\ v_{\varepsilon}(x) & \text { else }\end{cases}
$$

for $x_{p} \in \frac{1}{k} \mathbb{Z}^{2} \cap Q$ and interpolation ( $v_{\varepsilon}$ defined analogously to (10) with respect to $u_{\varepsilon}$ and $\mathbf{b}$ ). Then by (37) for $\varepsilon \leqq \varepsilon^{\prime}$,

$$
\left\|\tilde{y}^{\prime}-u_{\varepsilon}\right\| \leqq\left(c_{0}+\delta+o(1)\right) / k \leqq\left(c_{0}+2 \delta\right) / k,
$$

and since $k \Delta^{i} \tilde{y}^{\prime}$ is bounded,

$$
\left|f_{Q}\left(k \Delta^{i} \tilde{y}^{\prime}-\bar{b}^{i}\right) \mathrm{d} \rho\right|=\left|f_{Q} k \Delta^{i} \tilde{y}^{\prime} \mathrm{d} \rho-f_{Q} k \Delta^{i} \tilde{y} \mathrm{~d} \rho\right| \leqq \frac{C r_{Q}}{|k Q|} .
$$

Furthermore, by Corollary 1,

$$
\left|E(y)-E\left(y^{\prime}\right)\right| \leqq C r_{Q} .
$$

Invoking Lemma 10 (with $c_{0}$ replaced by $c_{0}+2 \delta$ and $c_{3}$ by $c_{0}$ ), we find a deformation $y^{\prime \prime}$ on $Q$ with

$$
\left\|\tilde{y}^{\prime \prime}-u_{\varepsilon}\right\| \leqq\left(c_{0}+2 \delta\right) / k \quad \text { and } \quad f_{Q} \Delta^{i} \tilde{y}^{\prime \prime} \mathrm{d} \rho=\bar{b}^{i}
$$

satisfying

$$
E\left(y^{\prime \prime}\right) \leqq E\left(y^{\prime}\right)+\frac{1}{\delta} \frac{C r_{Q}}{|k Q|}|k Q|
$$


(Note that the constant found in the proof of Lemma 10 by applying Lemma 8 isin the terminology of this lemma-Cl $-\mathrm{Cl}_{2} /\left(c_{0}-c_{3}\right)$. Here, this equals $C r_{Q} /|k Q| \delta$.) Finally, by Lemma 9 there is yet another deformation $y^{\prime \prime \prime}$ with

$$
\left\|\tilde{y}^{\prime \prime \prime}-u_{\varepsilon}\right\| \leqq c_{0} / k \text { and } f_{Q} \Delta^{i} \tilde{y}^{\prime \prime \prime}=\bar{b}^{i}
$$

and

$$
\left|E\left(y^{\prime \prime \prime}\right)-E\left(y^{\prime \prime}\right)\right| \leqq C \delta^{1 / 5}|k Q|
$$

Since $y^{\prime \prime \prime} \in \hat{\mathcal{N}}_{Q}\left(u_{\varepsilon}, \mathbf{b}\right)$ and $y \in \hat{\mathcal{N}}_{Q}(u, \mathbf{b})$ was arbitrary, we deduce from (38), (39) and (40)

$$
\inf _{y \in \hat{\mathcal{N}}_{Q}\left(u_{\varepsilon}, \mathbf{b}\right)} E(y) \leqq \inf _{y \in \hat{\mathcal{N}}_{Q}(u, \mathbf{b})} E(y)+C\left(\delta^{1 / 5}|k Q|+\frac{r_{Q}}{\delta}\right) .
$$

Interchanging the roles of $u$ and $u_{\varepsilon}$ (but defining $r_{Q}$ as before and only replacing $v_{\varepsilon}$ by $v$ in the definition of $y^{\prime}$ ) gives an analogous inequality.

To finish the proof, it remains to estimate $r_{Q}$. For $\delta$ small enough, the balls $B\left(x, \delta /\left(c_{2}+\bar{c}_{2}\right) k\right)$ with $x \in \frac{1}{k} \mathbb{Z}^{2}$ are disjoint. Since $|\nabla u| \leqq c_{2}$ and $|\nabla \bar{u}| \leqq \bar{c}_{2}$, we have $B\left(x, \delta /\left(c_{2}+\bar{c}_{2}\right) k\right) \cap\left(\mathcal{S}_{1} \backslash B\right)=\emptyset$ if $|u(x)-\bar{u}(x)|>\delta / k$. So indeed

$$
\frac{C \delta^{2}}{k^{2}} r_{Q} \leqq|B \cap Q|
$$

Now consider a partition of $\mathcal{S}_{1}$ with squares $\mathcal{D}_{j}$ of side-length $l_{3}$ and $R,|R| \leqq$ $2 l_{3}$ (see the next picture). Since $k l_{3} \rightarrow \infty$ and $k \varepsilon^{\prime} \rightarrow \infty$ (compare (36)), we may choose $\varepsilon=\varepsilon(k) \leqq \varepsilon^{\prime} \rightarrow 0$ with $k \varepsilon \rightarrow \infty$ as $k \rightarrow \infty$ such that eventually $l_{3} / \varepsilon \in \mathbb{N}$. This also induces a partition of $\mathcal{S}_{1}$ into squares $Q_{i}$ of side-length $\varepsilon$ and $R$ as in the picture below.
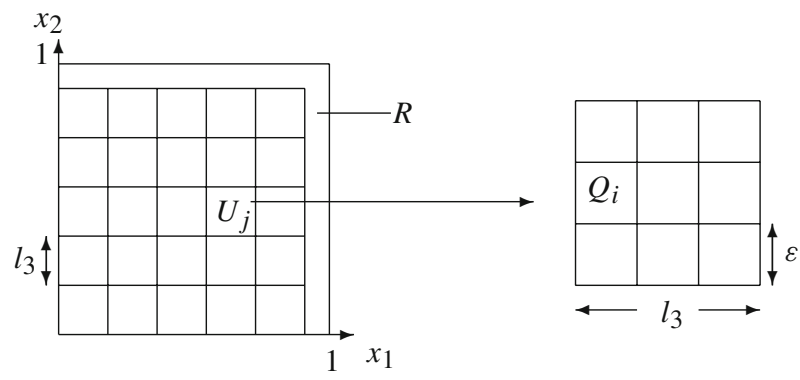

Proof of Theorem 3. Define $G$ to be the union of those $\mathcal{D}_{j}$ where $c_{1}-\delta<$ $s_{1}(\nabla \bar{u}) \leqq s_{2}(\nabla \bar{u})<c_{2}+\delta$. Since $\nabla \bar{u}$ is continuous, for $k$ large enough, $G \supset$ $\left\{x: c_{1} \leqq s_{1}(\nabla \bar{u}(x)) \leqq s_{2}(\nabla \bar{u}(x)) \leqq c_{2}\right\} \backslash R \supset \mathcal{S}_{1} \backslash(B \cup R)$, whence $|G| \geqq 1-|B \cup R| \geqq 1-\sigma-2 l_{3}$. 
Let $\mathcal{M}_{j}=y\left(\mathcal{L} \cap\left(k \mathcal{D}_{j} \times[0, h]\right)\right)$. It follows from Lemmas 7 and 6 that

$$
\begin{aligned}
\inf _{y \in \hat{\mathcal{N}}_{k}^{0, l_{3}}(u, \mathbf{b})} E(y)-\inf _{y \in \hat{\mathcal{N}}_{k}^{0, l_{3}}(u, \mathbf{b})} \sum_{\mathcal{D}_{j} \subset G} E\left(\mathcal{M}_{j}\right) \mid & \leqq C\left(\frac{k}{l_{3}}+k^{2} l_{3}+\frac{\left|\mathcal{S}_{1} \backslash G\right|}{l_{3}^{2}}\left(k l_{3}\right)^{2}\right) \\
& \leqq C k^{2}\left(\frac{1}{k l_{3}}+l_{3}+\sigma\right),
\end{aligned}
$$

where by definition of $\hat{\mathcal{N}}_{k}^{0, l_{3}}$,

$$
\inf _{y \in \hat{\mathcal{N}}_{k}^{0, l_{3}}(u, \mathbf{b})} \sum_{\mathcal{D}_{j} \subset G} E\left(\mathcal{M}_{j}\right)=\sum_{\mathcal{D}_{j} \subset G} \inf _{y \in \hat{\mathcal{N}}_{\mathcal{D}_{j}}(u, \mathbf{b})} E(y) .
$$

Now using Lemma 7 again,

$$
\inf _{y \in \hat{\mathcal{N}}_{\mathcal{D}_{j}}(u, \mathbf{b})} E(y)-\min \sum_{Q_{i} \subset \mathcal{D}_{j}} \inf _{y \in \hat{\mathcal{N}}_{Q_{i}}\left(u, \mathbf{b}_{j, i}\right)} E(y) \mid \leqq C \frac{k l_{3}^{2}}{\varepsilon},
$$

where the minimum is to be taken over admissible vectors $\mathbf{b}_{j, 1}, \ldots, \mathbf{b}_{j,\left(l_{3} / \varepsilon\right)^{2}}$ such that $\sum_{i} \frac{\rho\left(Q_{i}\right)}{\rho\left(\mathcal{D}_{j}\right)} \mathbf{b}_{j, i}=\mathbf{b}_{j}:=f_{\mathcal{D}_{j}} \overline{\mathbf{b}} \mathrm{d} \rho$.

Since $\nabla u_{\varepsilon} \rightarrow \nabla \bar{u}$ uniformly, we may choose matrices $A_{j}$ such that $\sup _{j} \mid A_{j}-$ $\nabla u_{\varepsilon} \mid=o(1)$ on $\mathcal{D}_{j}$. We now want to replace $u$ by $A_{j}$ in the right-hand side of (41). First replacing $u$ by $u_{\varepsilon}$ on $Q_{i}$ leads to an error bounded by $C\left(\delta^{1 / 5}\left|Q_{i}\right|+\mid B \cap\right.$ $\left.Q_{i} \mid / \delta^{3}\right) k^{2}$ by Lemma 16 . Now replacing $\nabla u_{\varepsilon}$ by $A_{j}$ leads to an additional error of order $o\left(\left|k Q_{i}\right|\right)$ because for matrices $A$,

$$
\inf _{y \in \hat{\mathcal{N}}_{Q_{i}}\left(A, \mathbf{b}_{j, i}\right)} E(y)=\varphi_{m}\left(A, \mathbf{b}_{j, i}\right) \nu\left|k Q_{i}\right|+\mathcal{O}(k \varepsilon),
$$

where $m$ is the integer part of $k \varepsilon$ or $k \varepsilon-1$ (use translational invariance), and $\left(\varphi_{k}\right)_{k}$ is equicontinuous by Proposition 2 , hence also $\left\{\varphi_{k}(\cdot, \mathbf{b}): k \in \mathbb{N}\right.$, $\mathbf{b}$ admissible $\}$ by compactness. It follows that

$$
\begin{aligned}
& \left|\inf _{y \in \hat{\mathcal{N}}_{k}^{0, l_{3}}(u, \mathbf{b})} E(y)-\sum_{\mathcal{D}_{j} \subset G}\left(\min \sum_{Q_{i} \subset \mathcal{D}_{j}} \inf _{y \in \hat{\mathcal{N}}_{Q_{i}}\left(A_{j}, \mathbf{b}_{j, i}\right)} E(y)\right)\right| \\
& \leqq C \sum_{Q_{i} \subset G}\left(\left(\delta^{1 / 5}+o(1)\right)\left|Q_{i}\right| k^{2}+\frac{\left|B \cap Q_{i}\right|}{\delta^{3}} k^{2}\right)+C k^{2}\left(\frac{1}{k \varepsilon}+l_{3}+\sigma\right) .
\end{aligned}
$$

Now reasoning as above, for $n=n(k)=\left\lfloor k l_{3}\right\rfloor$ or $\left\lfloor k l_{3}\right\rfloor-1$,

$$
\begin{aligned}
\min \sum_{Q_{i} \subset \mathcal{D}_{j}} \inf _{y \in \hat{\mathcal{N}}_{Q_{i}}\left(A_{j}, \mathbf{b}_{j, i}\right)} E(y) & =\inf _{y \in \hat{\mathcal{N}}_{\mathcal{D}_{j}}\left(A_{j}, \mathbf{b}_{j}\right)} E(y)+\mathcal{O}\left(k l_{3}^{2} / \varepsilon\right) \\
& =\varphi_{n}\left(A_{j}, \mathbf{b}_{j}\right) \nu\left|k \mathcal{D}_{j}\right|+\mathcal{O}\left(k l_{3}^{2} / \varepsilon+k l_{3}\right) .
\end{aligned}
$$


Summarizing (using Theorem 2 to choose $n=\left\lfloor k l_{3}\right\rfloor$ uniquely), we obtain

$$
\begin{aligned}
\left|\frac{1}{\nu k^{2}} \inf _{y \in \hat{\mathcal{N}}_{k}^{0, l_{3}}(u, \mathbf{b})} E(y)-\sum_{\mathcal{D}_{j} \subset G} \varphi_{n}\left(A_{j}, \mathbf{b}_{j}\right)\right| \mathcal{D}_{j}|| & \leqq C\left(\delta^{1 / 5}+|B| / \delta^{3}+\sigma+o(1)\right) \\
& \leqq C\left(\delta^{1 / 5}+\sigma / \delta^{3}\right) .
\end{aligned}
$$

Let $\left.\Omega=\left\{x: c_{1}-\delta<s_{1}(\nabla \bar{u}) \leqq s_{2}(\nabla \bar{u})<c_{2}+\delta\right)\right\}$. Then $\lim _{\inf _{k}} G \supset \Omega$. The piecewise linear respectively constant approximations $A_{j}$ respectively $\mathbf{b}_{j}$ converge to $\nabla \bar{u}$ uniformly respectively to $\mathbf{b}$ boundedly in measure. (This is not hard to see: approximate $\mathbf{b}$ by continuous functions in measure.) So we deduce from Lemma 17 and Theorem 2

$$
\sum_{\mathcal{D}_{j} \subset G} \varphi_{n}\left(A_{j}, \mathbf{b}_{j}\right)\left|\mathcal{D}_{j} \cap \Omega\right| \rightarrow \int_{\Omega} \varphi(\nabla \bar{u}, \mathbf{b}) .
$$

Since $\mathcal{S}_{1} \backslash \Omega \subset B,|B| \leqq \sigma$, and $\varphi_{n}, \varphi$ are uniformly bounded on compact subsets of admissible matrices, we finally obtain that

$$
\limsup _{k \rightarrow \infty}\left|\frac{1}{\nu k^{2}} \inf _{y \in \hat{\mathcal{N}}^{0, l_{3}}(u, \mathbf{b})} E(y)-\int_{\mathcal{S}_{1}} \varphi(\nabla u, \mathbf{b})\right| \leqq C\left(\delta^{1 / 5}+\sigma / \delta^{3}\right) .
$$

Now let $\sigma \rightarrow 0, \delta \rightarrow 0$.

Remark. Assuming regularity for $\nabla u, \mathbf{b}$, for example to lie in some Hölder class, the above proof gives explicit error estimates.

Lemma 17. Let $\Omega \subset \mathbb{R}^{n}$ be of finite measure, $v_{k}: \Omega \rightarrow K, k=1,2, \ldots$, measurable, $K$ some compact subset of $\mathbb{R}^{m}$ and $f_{k}: K \rightarrow \mathbb{R}$ such that $f_{k} \circ v_{k}$ is integrable. Furthermore suppose that $\Omega_{k} \subset \Omega$ is measurable with $\left|\Omega \backslash \Omega_{k}\right| \rightarrow 0$ as $k \rightarrow \infty$. If $f_{k} \rightarrow f$ uniformly on $K, f: K \rightarrow \mathbb{R}$ continuous and $v_{k} \rightarrow v$ in measure, then

$$
\lim _{k \rightarrow \infty} \int_{\Omega_{k}} f_{k}\left(v_{k}\right)=\int_{\Omega} f(v)
$$

The proof of this lemma is a straightforward $\varepsilon / 4$-argument.

\subsection{Extension to infinite pair-interactions}

We will now prove Theorem 4. For this paragraph we assume that Proposition 5 is already proven.

Suppose $E$ is given as in (16). For given $\delta$ we choose

$$
E_{\delta}(y)=\frac{1}{2} \sum_{i \neq j} W_{\delta}\left(\left|y_{i}-y_{j}\right|\right)+E_{0}(y)
$$


where $W_{\delta} \leqq W$ satisfies the hypotheses of Proposition 5, and

$$
W_{\delta}(r)=W(r) \text { for } r \geqq \delta, \quad W_{\delta}(r) \geqq \min _{0<s \leqq \delta} W(s) \text { for } r \leqq \delta .
$$

Proposition 5 implies that $E_{\delta}$ is an admissible energy function. If $\delta$ is small enough, we may assume that $W(r)>0$ for $r \leqq \delta$. Note also that there exists $C=C(\delta, c)$ such that for all $z \in \mathcal{L}_{k}$ and $y$ with $\|\tilde{y}-u\| \leqq c / k$ ( $u$ admissible $)$

$$
\sum_{\substack{x \in \mathcal{L}_{k} \\ x \neq z}}\left|W_{\delta}(|y(x)-y(z)|)\right| \leqq C .
$$

This follows from Lemma 6 (with $\mathcal{K}_{2}=\{z\}$ ) applied to the pair potential given by $\left|W_{\delta}\right|$.

Definition 4. Let $\delta>0$, and suppose $y$ is some deformation. We call $\left(y_{i}, y_{j}\right)$, $i \neq j$, a $\delta$-critical bond if $\left|y_{i}-y_{j}\right|<\delta$. We say that $y$ satisfies a minimal distance hypothesis with $\delta$ if it does not contain $\delta$-critical bonds.

Lemma 18. Suppose $y$ is a deformation with $\|\tilde{y}-u\| \leqq c / k, u$ admissible.

(i) The number of atoms in a ball $B$ of radius $R$ is bounded by a constant $n=n(R)$.

(ii) There exists $C>0$ such that if $(y(x), y(z))$ is 1 -critical, then $|x-z| \leqq C$.

Proof. (i) Suppose $y_{i}=y\left(x_{i}\right) \in B$. Choose $\delta=2 C_{3} / C_{1}$ as in the proof of Lemma 6. Then for $|x-z| \geqq \delta$ we have $\frac{C_{1}}{2}|x-z| \leqq|y(x)-y(z)|$ and thus

$$
|y(x)-y(z)| \leqq 2 R \Rightarrow|x-z| \leqq \delta \text { or }|x-z| \leqq 4 R / C_{1} .
$$

So \#\{j: $\left.y_{j} \in B\right\} \leqq \#\left\{j:\left|x_{i}-x_{j}\right| \leqq \max \left\{2 C_{3} / C_{1}, 4 R / C_{1}\right\}\right\}=: n(R)$.

(ii) Just note that by Lemma 1 (ii), $|x-z| \leqq\left(|y(x)-y(z)|+C_{3}\right) / C_{1}$.

We will prove Theorem 4 by reducing to the case of admissible energy functions already treated. The main point is to show that we may impose an additional minimal distance hypothesis on the deformations. To this end, for given $y$ we have to find a new configuration $y^{\prime}$ satisfying this hypothesis whose energy does not exceed $E(y)$ too much. The main difficulty comes again from the condition on local spatial averages.

Let $(A, \mathbf{b}) \in \mathcal{A}_{\text {hom. }}$. As in the proof of Lemma 9 we choose

$$
b^{0} \in \underset{b^{0}}{\operatorname{argmin} \max }\left\{\max _{1 \leqq i \leqq \nu-1}\left|b^{i}-b^{0}\right|,\left|b^{0}\right|\right\}
$$

and set

$$
B^{i}=b^{i-1}-b^{0}, \quad i=2, \ldots, v, \quad B^{1}=-b^{0} .
$$

We will first assume that there is some $\theta>0$ such that, if $\left|B^{i}\right|,\left|B^{j}\right| \geqq c_{0}-\theta$ and there is $z \in \mathbb{Z}^{2}$ with $\left|B^{i}-B^{j}-A z\right| \leqq \theta$, then $i=j$ and $z=0$. 
Now suppose $y \in \hat{\mathcal{N}}_{Q}^{l_{2}, l_{3}}(A, \mathbf{b})$ where $Q$ is a square of side-length $l_{3} \gg 1 / k$. We construct a new deformation $y^{\prime}: \mathcal{L} \cap k Q \times[0, h] \rightarrow \mathbb{R}^{3}$ in two steps. Let

$$
0<\delta_{1}<\delta_{1}^{\prime}<\frac{\delta_{2}}{6 n\left(2 \delta_{2}\right)}, \quad 3 \delta_{2}<\delta_{2}^{\prime} \leqq \min \left\{1, c_{1}\right\}
$$

be small enough $\left(n\left(2 \delta_{2}\right)\right.$ as in the previous lemma, $\left.c_{1}=s_{1}(A)\right)$.

Step 1. We first derive an intermediate deformation from $y$ by successively moving the atoms around. At each intermediate step we are dealing with deformations $\hat{y}$ such that $\|\hat{y}-A\| \leqq c_{0}$, so Lemma 18 is applicable.

We will reorder layer by layer of the film starting with $i=0$. Suppose the first $i-1$ layers and the first $m$ atoms of the $i$ th layer $y(\cdot, i)$ have been reordered in the way described below. Let $x=\left(x_{1}, x_{2}, i\right)$ be the $(m+1)$ th atom. We reorder in the following way:

If $y(x)$ has a distance greater than or equal to $\delta_{1}$ to all the other atomic positions, it remains unchanged.

Now suppose $y(x)$ takes part in a $\delta_{1}$-critical bond. If there exists another atom at $y\left(x^{\prime}\right), x^{\prime}=\left(x_{p}^{\prime}, i\right)$, and a unit vector $e \in \mathbb{R}^{3}$ such that

$$
\left|y(x)+r e-A x_{p}\right| \leqq c_{0} \quad \text { and } \quad\left|y\left(x^{\prime}\right)-r e-A x_{p}^{\prime}\right| \leqq c_{0}
$$

for $0 \leqq r \leqq \delta_{2}$, then both of the atoms $y(x)$ and $y\left(x^{\prime}\right)$ will be moved in opposite directions. Let $L=\left\{y(x)+r e: 0 \leqq r \leqq \delta_{2}\right\}, L^{\prime}=\left\{y\left(x^{\prime}\right)-r e: 0 \leqq r \leqq \delta_{2}\right\}$.

Claim. There are points $Y(x) \in L, Y\left(x^{\prime}\right) \in L^{\prime}$ with

$$
y(x)+y\left(x^{\prime}\right)=Y(x)+Y\left(x^{\prime}\right)
$$

such that

$$
\left|Y(x)-Y\left(x^{\prime}\right)\right|,|Y(x)-y(z)|,\left|Y\left(x^{\prime}\right)-y(z)\right| \geqq \delta_{1}^{\prime}
$$

for all $z \in \mathcal{L}_{k}, z \neq x, x^{\prime}$.

Proof of the claim. Let $B, B^{\prime}$ be balls of radius $2 \delta_{2}$ centered at $y(x)$ respectively $y\left(x^{\prime}\right)$. Clearly, $\operatorname{dist}(z, \bar{z}) \geqq \delta_{2}>\delta_{1}$ if $z \in L$ and $\bar{z} \notin B$ (respectively if $z \in L^{\prime}$ and $\left.\bar{z} \notin B^{\prime}\right)$. By the preceding lemma there are at most $n\left(2 \delta_{2}\right)$ atoms in these balls. Consider balls $B_{l}$, respectively $B_{l^{\prime}}^{\prime}$ with radius $\delta_{1}^{\prime}$ around the atoms in the balls $B$, respectively $B^{\prime}$. Since by assumption $\delta_{1}^{\prime}<\delta_{2} / 6 n\left(2 \delta_{2}\right)$ we get $\left(\mathcal{H}^{1}\right.$ denoting one-dimensional Hausdorff measure)

$$
\mathcal{H}^{1}\left(L \backslash \bigcup_{l} B_{l}\right) \geqq 2 \delta_{2} / 3, \quad \mathcal{H}^{1}\left(L^{\prime} \backslash \bigcup_{l^{\prime}} B_{l^{\prime}}^{\prime}\right) \geqq 2 \delta_{2} / 3 .
$$

Since the mapping $L \rightarrow L^{\prime}$ with $z \mapsto z^{\prime}$ such that $z+z^{\prime}=y(x)+y\left(x^{\prime}\right)$, that is, $z^{\prime}=y(x)+y\left(x^{\prime}\right)-z$ is isometric, we find that

$$
\mathcal{H}^{1}\left(\left\{z \in L \backslash \bigcup_{l} B_{l}: z^{\prime} \notin \bigcup_{l^{\prime}} B_{l^{\prime}}^{\prime}\right\}\right) \geqq \delta_{2} / 3 .
$$


Noting that $\left|z-z^{\prime}\right| \leqq \delta_{1}^{\prime} \Rightarrow\left|y(x)+y\left(x^{\prime}\right)-2 z\right| \leqq \delta_{1}^{\prime}$, we also get that

$$
\mathcal{H}^{1}\left(\left\{z \in L:\left|z-z^{\prime}\right| \leqq \delta_{1}^{\prime}\right\}\right) \leqq \delta_{1}^{\prime}
$$

so we have shown that

$$
\mathcal{H}^{1}\left(\left\{z \in L \backslash \bigcup_{l} B_{l}: z^{\prime} \notin \bigcup_{l^{\prime}} B_{l^{\prime}}^{\prime},\left|z-z^{\prime}\right| \geqq \delta_{1}^{\prime}\right\}\right) \geqq \delta_{2} / 3-\delta_{1}^{\prime}>0 .
$$

In particular, there exist points $Y(x)=z \in L, Y\left(x^{\prime}\right)=z^{\prime} \in L^{\prime}$ as claimed.

We now update the deformation by replacing $y(x)$ by $Y(x)$ and $y\left(x^{\prime}\right)$ by $Y\left(x^{\prime}\right)$. If each atom has been considered this way we arrive at a new configuration again denoted $y$. We repeat the process until there are no more $\delta_{1}$-critical bonds that can be removed this way. (There may still be $\delta_{1}$-critical bonds left.)

Step 2. If there are no more $\delta_{1}$-critical bonds, we are done. If there still are, using the new configuration constructed in Step 1 (again called $y$ ), we now construct $y^{\prime}$. Suppose $y(x)$ takes part in a $\delta_{1}$-critical bond. Then it is not possible to find another atom in the same film layer and the unit vector $e$ as described above. But then for all $x^{\prime} \in \mathcal{L} \cap(k Q \times[0, h])$ with $x_{3}=x_{3}^{\prime}$,

$$
\left|y\left(x^{\prime}\right)-A x_{p}^{\prime}-\left[y(x)-A x_{p}\right]\right| \leqq \delta_{2},
$$

for otherwise we could define

$$
e=\frac{y\left(x^{\prime}\right)-A x_{p}^{\prime}-\left[y(x)-A x_{p}\right]}{\left|y\left(x^{\prime}\right)-A x_{p}^{\prime}-\left[y(x)-A x_{p}\right]\right|} .
$$

In particular, there are no $\delta_{1}$-critical bonds within the set $y(k Q \times\{i\})$. (If $\left(y\left(x^{\prime}\right)\right.$, $\left.y\left(x^{\prime \prime}\right)\right)$ was critical, then by $\left|y\left(x^{\prime}\right)-A x_{p}^{\prime}-\left[y\left(x^{\prime \prime}\right)-A x_{p}^{\prime \prime}\right]\right| \leqq 2 \delta_{2}$ we would have $\left|A x_{p}^{\prime}-A x_{p}^{\prime \prime}\right| \leqq 2 \delta_{2}+\delta_{1}<c_{1}$ in contradiction to (47).)

Now suppose $\left(y(x), y\left(x^{\prime}\right)\right)$ is critical where $x^{\prime}=\left(x_{p}^{\prime}, i^{\prime}\right), i^{\prime} \neq i$. Then again, as in (48), for all $z_{p}, z_{p}^{\prime} \in \mathbb{Z}^{2} \cap k Q$,

$$
\left|y\left(z_{p}, i\right)-A z_{p}-\left[y(x)-A x_{p}\right]\right| \leqq \delta_{2}
$$

and

$$
\left|y\left(z_{p}^{\prime}, i^{\prime}\right)-A z_{p}^{\prime}-\left[y\left(x^{\prime}\right)-A x_{p}^{\prime}\right]\right| \leqq \delta_{2} .
$$

In particular for $z_{p}^{\prime}-z_{p}=x_{p}^{\prime}-x_{p}$,

$$
\left|y\left(z_{p}^{\prime}, i^{\prime}\right)-A z_{p}^{\prime}-\left[y\left(x^{\prime}\right)-A x_{p}^{\prime}\right]-\left(y\left(z_{p}, i\right)-A z_{p}-\left[y(x)-A x_{p}\right]\right)\right| \leqq 2 \delta_{2},
$$

so

$$
\begin{aligned}
\left|y\left(z_{p}^{\prime}, i^{\prime}\right)-y\left(z_{p}, i\right)\right| & \leqq\left|y(x)-y\left(x^{\prime}\right)+A x_{p}^{\prime}-A x_{p}+A z_{p}-A z_{p}^{\prime}\right|+2 \delta_{2} \\
& \leqq \delta_{1}+2 \delta_{2} \leqq 3 \delta_{2} .
\end{aligned}
$$


Since $\left|x_{p}-x_{p}^{\prime}\right| \leqq C$ (compare Lemma 18 (ii)), we find (up to a constant boundary layer) at least one $3 \delta_{2}$-critical bond per atom of the $i$ th layer. If this case occurs, that is, we have more than $\left(\mathrm{kl}_{3}\right)^{2}-\mathrm{Ckl}_{3} 3 \delta_{2}$-critical bonds, we reorder all the atoms in $k Q \times[0, h]$, first by placing atom $x$ at position $V(x)$ ( $V$ such that $\tilde{V}=v$, compare (10)). Now suppose $\delta_{2}^{\prime}$ is small enough. Then since $\left|B^{i}\right|<c_{0}-\theta$ or $\left|B^{j}\right|<c_{0}-\theta$ if $\left|B^{i}-B^{j}-A z\right| \leqq \theta$ for $i \neq j$ and some $z \in \mathbb{Z}^{2}$, we can eliminate all $3 \delta_{2}$-critical bonds as in Step 1, arriving at a new deformation $y$ such that no atom in $y(k Q \times[0, h])$ takes part in a $\delta_{2}^{\prime}$-critical bond.

Lemma 19. Suppose $\left|B^{i}\right|=\left|B^{j}\right|=c_{0}$ and $B^{i}-B^{j} \in A \mathbb{Z}^{2}$ only for $i=j$. (So $\theta$ as above can be chosen.) There are $0<\delta_{1}, \delta_{1}^{\prime}, \delta_{2}, \delta_{2}^{\prime}$ (only depending on $W, E_{0}$, and $\theta$ ) such that (47) holds, and (compare (42)) for all $y \in \hat{\mathcal{N}}_{Q}^{l_{2}, l_{3}}(A, \mathbf{b})$

$$
E_{\delta_{1}}\left(y^{\prime}\right) \leqq E_{\delta_{1}}(y)
$$

where $y^{\prime}$ is derived from $y$ as described above. In fact, $y^{\prime} \in \hat{\mathcal{N}}_{Q}^{l_{2}, l_{3}}(A, \mathbf{b})$ with $E\left(y^{\prime}\right) \leqq E_{\delta_{1}}(y)$.

Proof. We prove that each step of the above construction lowers energy. Assume $\delta_{2}^{\prime}$ is so small that $W(r) \geqq 0$ on $\left(0, \delta_{2}^{\prime}\right]$ and thus also $W_{\delta} \geqq 0$ on $\left(0, \delta_{2}^{\prime}\right]$ for $\delta \leqq \delta_{2}^{\prime}$ (compare (43)). Suppose $\hat{y}$ and $\hat{y}^{\prime}$ are intermediate configurations in Step 1 above and $\hat{y}^{\prime}$ arises from $\hat{y}$ by moving the atoms $x$ and $x^{\prime}$. By Corollary 1 , changing the position of two atoms yields an energy error in $E_{0}$ bounded by some constant $C$. For given (small) $\delta_{1}^{\prime}$ choose $\delta_{1}$ so small that

$$
W_{\delta_{1}}(r)>C+4 \sup _{\|y-A\| \leqq c_{0}} \sup _{z} \sum_{z^{\prime} \neq z}\left|W_{\delta_{1}^{\prime}}\left(\left|y\left(z^{\prime}\right)-y(z)\right|\right)\right|
$$

for all $r \leqq \delta_{1}$ (which is possible by (44) and (43)). Now $y(x)$ having a critical bond of length $r<\delta_{1}$,

$$
\begin{aligned}
E_{\delta_{1}}(\hat{y})-E_{\delta_{1}}\left(\hat{y}^{\prime}\right) & \\
= & \sum_{z \neq x, x^{\prime}} W_{\delta_{1}}(|\hat{y}(z)-\hat{y}(x)|)+\sum_{z \neq x, x^{\prime}} W_{\delta_{1}}\left(\left|\hat{y}(z)-\hat{y}\left(x^{\prime}\right)\right|\right) \\
& -\sum_{z \neq x, x^{\prime}} W_{\delta_{1}}\left(\left|\hat{y}^{\prime}(z)-\hat{y}^{\prime}(x)\right|\right)-\sum_{z \neq x, x^{\prime}} W_{\delta_{1}}\left(\left|\hat{y}^{\prime}(z)-\hat{y}^{\prime}\left(x^{\prime}\right)\right|\right) \\
& +W_{\delta_{1}}\left(\left|\hat{y}(x)-\hat{y}\left(x^{\prime}\right)\right|\right)-W_{\delta_{1}}\left(\left|\hat{y}^{\prime}(x)-\hat{y}^{\prime}\left(x^{\prime}\right)\right|\right)+C \\
\geqq & \sum_{\substack{z \neq x, x^{\prime} \\
|\hat{y}(z)-\hat{y}(x)| \geqq \delta_{1}^{\prime}}} W_{\delta_{1}^{\prime}}(|\hat{y}(z)-\hat{y}(x)|)+\sum_{\substack{z \neq x, x^{\prime} \\
\left|\hat{y}(z)-\hat{y}\left(x^{\prime}\right)\right| \geqq \delta_{1}^{\prime}}} W_{\delta_{1}^{\prime}}\left(\left|\hat{y}(z)-\hat{y}\left(x^{\prime}\right)\right|\right) \\
& -\sum_{z \neq x, x^{\prime}} W_{\delta_{1}^{\prime}}\left(\left|\hat{y}^{\prime}(z)-\hat{y}^{\prime}(x)\right|\right)-\sum_{z \neq x, x^{\prime}} W_{\delta_{1}^{\prime}}\left(\left|\hat{y}^{\prime}(z)-\hat{y}^{\prime}\left(x^{\prime}\right)\right|\right) \\
& +W_{\delta_{1}}(r)-W_{\delta_{1}^{\prime}}\left(\left|\hat{y}^{\prime}(x)-\hat{y}^{\prime}\left(x^{\prime}\right)\right|\right)-C \\
\geqq & 0 .
\end{aligned}
$$


Now consider the construction of $y^{\prime}$ in Step 2 and suppose there are $\left(k l_{3}\right)^{2}-$ $\mathrm{Ckl}_{3}>\left(\left\lceil k l_{3}\right\rceil+1\right)^{2} / 23 \delta_{2}$-critical bonds between the $i$ th and $i^{\prime}$ th layer in $y(\mathcal{L} \cap$ $(k Q \times[0, h]))$. The energy change due to the $E_{0}$-term is bounded by $C\left(k l_{3}\right)^{2}$. So if for given $\delta_{2}^{\prime}, \delta_{1}$ and $\delta_{2}$ are chosen such that

$$
W_{\delta_{1}}(r)>2 C+\sup _{\|y-A\| \leqq c_{0}} \sup _{x} 2 v \sum_{x^{\prime} \neq x}\left|W_{\delta_{2}^{\prime}}\left(\left|y\left(x^{\prime}\right)-y(x)\right|\right)\right|
$$

for all $r \leqq 3 \delta_{2}$, then

$$
\begin{aligned}
& E_{\delta_{1}}(y)-E_{\delta_{1}}\left(y^{\prime}\right) \\
&=\frac{1}{2} \sum_{x^{\prime} \neq x} W_{\delta_{1}}\left(\left|y\left(x^{\prime}\right)-y(x)\right|\right)-\frac{1}{2} \sum_{x^{\prime} \neq x} W_{\delta_{1}}\left(\left|y^{\prime}\left(x^{\prime}\right)-y^{\prime}(x)\right|\right)+E_{0}(y)-E_{0}\left(y^{\prime}\right) \\
& \geqq \frac{1}{2} \sum_{\substack{x^{\prime} \neq x \\
\left|y(x)-y\left(x^{\prime}\right)\right| \leqq 3 \delta_{2}}} W_{\delta_{1}}\left(\left|y(x)-y\left(x^{\prime}\right)\right|\right)+\frac{1}{2} \sum_{\substack{x^{\prime} \neq x \\
\left|y(x)-y\left(x^{\prime}\right)\right|>\delta_{2}^{\prime}}} W_{\delta_{2}^{\prime}}\left(\left|y\left(x^{\prime}\right)-y(x)\right|\right) \\
& \quad-\frac{1}{2} \sum_{x^{\prime} \neq x} W_{\delta_{2}^{\prime}}\left(\left|y^{\prime}\left(x^{\prime}\right)-y^{\prime}(x)\right|\right)+E_{0}(y)-E_{0}\left(y^{\prime}\right) \\
& \geqq \frac{\left(\left\lceil k l_{3}\right\rceil+1\right)^{2}}{2}\left(\cos ^{2 C}+2 v \sup _{\|y-A\| \leqq c_{0}} \sup _{x} \sum_{x^{\prime} \neq x}\left|W_{\delta_{2}^{\prime}}\left(\left|y\left(x^{\prime}\right)-y(x)\right|\right)\right|\right) \\
&-\frac{1}{2} v\left(\left\lceil k l_{3}\right\rceil+1\right)^{2} \sup _{x} \sum_{x^{\prime} \neq x}\left|W_{\delta_{2}^{\prime}}\left(\left|y\left(x^{\prime}\right)-y(x)\right|\right)\right| \\
&-\frac{1}{2} v\left(\left\lceil k l_{3}\right\rceil+1\right)^{2} \sup _{x} \sum_{x^{\prime} \neq x}\left|W_{\delta_{2}^{\prime}}\left(\left|y^{\prime}\left(x^{\prime}\right)-y^{\prime}(x)\right|\right)\right|-C\left(k l_{3}\right)^{2} \\
& \geqq 0 .
\end{aligned}
$$

Clearly, $\left\|\tilde{y}^{\prime}-A\right\|_{\infty} \leqq c_{0} / k$. Since Step 1 leaves $f_{Q} k \Delta^{i} \tilde{y} \mathrm{~d} \rho$ unchanged and $k \Delta^{i} v=\bar{b}^{i}$, we have indeed $y^{\prime} \in \hat{\mathcal{N}}_{Q}^{l_{2}, l_{3}}(A, \mathbf{b})$. By construction $y^{\prime}$ satisfies a minimal distance hypothesis with $\delta_{1}$, so $E_{\delta_{1}}\left(y^{\prime}\right)=E\left(y^{\prime}\right)$.

Write $\hat{\mathcal{N}}_{k, c_{0}}^{l_{2}, l_{3}}(u, \mathbf{b})$ to highlight the dependence of the weak neighborhoods on $c_{0}$. In the non-homogeneous setting we will need the following

Lemma 20. Let $\delta_{2}>0$. For all $y \in \hat{\mathcal{N}}_{k, c_{0}-\delta_{2}}^{l_{2}, l_{3}}(u, \mathbf{b})$ there exists $y^{\prime} \in \hat{\mathcal{N}}_{k, c_{0}}^{l_{2}, l_{3}}(u, \mathbf{b})$ with $E\left(y^{\prime}\right) \leqq E_{\delta_{1}}(y)$ if $\delta_{1}$ is sufficiently small.

Proof. Derive $y^{\prime}$ from $y$ similarly as in Step 1 of the procedure described above applied to the sets $\mathcal{L} \cap\left(\mathcal{D}_{j} \times[0, h]\right)$ for $j=1, \ldots, N$ individually. If the unit vector $e$ is taken to be the same for each atom to be considered, we may choose $x^{\prime}$ to be the next (the $(m+2)$ th) lattice point, respectively the first if $x$ was the last one of the points in $k \mathcal{D}_{j} \cap \mathbb{Z}^{2}$. Clearly, $y^{\prime} \in \hat{\mathcal{N}}_{k, c_{0}}^{l_{2}, l_{3}}(u, \mathbf{b})$. As before, we see that $E\left(y^{\prime}\right) \leqq E_{\delta_{1}}(y)$. 
We first analyze $\varphi$. The first part of Theorem 4 is contained in the following proposition.

Proposition 3. Suppose A and $\mathbf{b}$ are admissible. Then the limit

$$
\varphi(A, \mathbf{b})=\lim _{k \rightarrow \infty} \frac{1}{v k^{2}} \inf _{y \in \hat{\mathcal{N}}_{k}^{0,1}(A, \mathbf{b})} E(y)
$$

exists in $(-\infty, \infty], \varphi$ is continuous on $\mathcal{A}_{\mathrm{hom}}$ (as a function with values in $\mathbb{R} \cup\{\infty\}$ ), and $\varphi(A, \mathbf{b})=\infty$ if and only if there are $z \in \mathbb{Z}^{2}, i \neq j \in\{1, \ldots, v\}$ such that $B^{i}-B^{j}=A z$ and $\left|B^{i}\right|=\left|B^{j}\right|=c_{0}$. (B $B^{i}$ as in (46), (45).)

Furthermore, $\varphi_{\delta}$ denoting the limiting energy density corresponding to $E_{\delta}$ (compare (42)) $\varphi_{\delta} \nearrow \varphi$ pointwise on $\mathcal{A}_{\mathrm{hom}}$ as $\delta \searrow 0$.

Proof. Suppose first that $B^{i}-B^{j} \notin A \mathbb{Z}^{2}$ if $\left|B^{i}\right|=\left|B^{j}\right|=c_{0}, i \neq j$. By Lemma 19,

$$
\inf _{y \in \hat{\mathcal{N}}_{k}^{0,1}(A, \mathbf{b})} E(y) \leqq \inf _{y \in \hat{\mathcal{N}}_{k}^{0,1}(A, \mathbf{b})} E_{\delta_{1}}(y)
$$

for $\delta_{1}$ sufficiently small. But $E_{\delta_{1}} \leqq E$, so the reverse inequality it true, too. We may therefore replace $E$ by $E_{\delta_{1}}$ and infer from Theorem 2 that $\varphi(A, \mathbf{b})$ exists in $\mathbb{R}$, and $\varphi$ is continuous at these $A, \mathbf{b}$.

For $0<\theta \leqq 1$ given, suppose now there are $z \in \mathbb{Z}^{2}$ and $i \neq j$ such that $\left|B^{i}\right|,\left|B^{j}\right| \geqq c_{0}-\theta,\left|B^{i}-B^{j}-A z\right| \leqq \theta$. We define $Y^{i}$ and $\overline{Y^{i}}$ as in the proof of Lemma 9. There it was shown that for $\left|B^{i_{0}}\right| \geqq c_{0}-\theta$ we have (compare (25) and (26) with $\varepsilon^{\prime}=\theta$ and $\delta=0$ )

$$
\left|\overline{Y^{i_{0}}}-B^{i_{0}}\right| \leqq C \sqrt{\theta}, \quad \sum_{x \in \frac{1}{k} \mathbb{Z}^{2} \cap \mathcal{S}_{1}}\left|Y^{i_{0}}(x)-\overline{Y^{i_{0}}}\right| \leqq C k^{2} \sqrt[4]{\theta} .
$$

For $\left|B^{i}-B^{j}-A z\right| \leqq \theta$ this implies (modulo boundary terms)

$$
\begin{aligned}
& \sum_{x \in \frac{1}{k} \mathbb{Z}^{2} \cap \mathcal{S}_{1}} k|\tilde{y}(x, i-1)-\tilde{y}(x+z / k, j-1)| \\
= & \sum_{x \in \frac{1}{k} \mathbb{Z}^{2} \cap \mathcal{S}_{1}}\left|Y^{i}(x)-Y^{j}(x+z / k)-A z\right| \\
\leqq & \sum_{x \in \frac{1}{k} \mathbb{Z}^{2} \cap \mathcal{S}_{1}}\left|Y^{i}(x)-\overline{Y^{i}}\right|+\left|\overline{Y^{i}}-B^{i}\right|+\left|B^{i}-B^{j}-A z\right| \\
& +\left|B^{j}-\overline{Y^{j}}\right|+\left|\overline{Y^{j}}-Y^{j}(x)\right| \\
\leqq & C k^{2} \sqrt[4]{\theta}
\end{aligned}
$$

so the number of $4 C \sqrt[4]{\theta}$-critical bonds is at least $k^{2} / 2$. This holds for all $y \in \hat{\mathcal{N}}_{k}^{0,1}(A, \mathbf{b})$, so by (29),

$$
\varphi(A, \mathbf{b}):=\liminf _{k \rightarrow \infty} \varphi_{k}(A, \mathbf{b}) \geqq \frac{1}{2 v} \inf _{0<s \leqq 4 C \sqrt[4]{\theta}} W(s)-C .
$$


Since the right-hand side of this inequality converges to $\infty$ as $\theta \rightarrow 0$, the first part of the proposition is proven.

It remains to prove that $\varphi_{\delta} \nearrow \varphi$. This is clear on the set $\left\{\mathbf{b}: B^{i}-B^{j} \notin\right.$ $A \mathbb{Z}^{2}$ for $\left.i \neq j\right\}$ since there $\varphi=\varphi_{\delta}$ for $\delta$ sufficiently small as just shown. If $B^{i}-B^{j} \in A \mathbb{Z}^{2}$, then the above calculations show that

$$
\varphi(A, \mathbf{b}) \geqq \varphi_{\delta}(A, \mathbf{b}) \geqq \frac{1}{2 v} W_{\delta}(0)-C \rightarrow \infty \quad \text { as } \quad \delta \rightarrow 0 .
$$

For the inhomogeneous case define

$$
\begin{aligned}
M^{\theta}:= & \left\{x \in \mathcal{S}_{1}: \exists z \in \mathbb{Z}^{2}, i \neq j \in\{1, \ldots, v\} \text { s.t. }\left|B^{i}(x)\right|,\left|B^{j}(x)\right| \geqq c_{0}-\theta,\right. \\
& \left.\left|B^{i}(x)-B^{j}(x)-\nabla u(x) z\right| \leqq \theta\right\}
\end{aligned}
$$

for $(u, \mathbf{b})$ admissible, where $b^{0}, B^{i}$ satisfy (45) and (46) pointwise.

Proof of Theorem 4. By Proposition 3 it remains to prove upper and lower bounds for general admissible $(u, \mathbf{b})$. This is done in four steps:

1 . It is easy to get lower bounds. Since $E \geqq E_{\delta_{1}}$, we have for $y^{(k)} \rightarrow(u, \mathbf{b})$,

$$
\liminf _{k \rightarrow \infty} \frac{1}{\nu k^{2}} E\left(y^{(k)}\right) \geqq \liminf _{k \rightarrow \infty} \frac{1}{\nu k^{2}} E_{\delta_{1}}\left(y^{(k)}\right) \geqq \int_{\mathcal{S}_{1}} \varphi_{\delta_{1}}(\nabla u, \mathbf{b})
$$

for all $\delta_{1}>0$. Now by Proposition $3 \varphi_{\delta_{1}} \nearrow \varphi$ pointwise as $\delta_{1} \rightarrow 0$, so

$$
\liminf _{k \rightarrow \infty} \frac{1}{v k^{2}} E\left(y^{(k)}\right) \geqq \int_{\mathcal{S}_{1}} \varphi(\nabla u, \mathbf{b})
$$

by monotone convergence.

2. First suppose that $\left|B^{i}(x)\right| \leqq c_{0}-\theta$ almost everywhere for some $\theta>0$. Then by Lemma 20 for appropriately chosen $\delta_{1}, \delta_{2}$ small,

$$
\inf _{y \in \hat{\mathcal{N}}_{k, c_{0}}^{l_{2}, l_{3}}(u, \mathbf{b})} E(y) \leqq \inf _{y \in \hat{\mathcal{N}}_{k, c_{0}-\delta_{2}}^{l_{2}, l_{3}}}(u, \mathbf{b})
$$

Now by Theorems 2 and 3 (see also Corollary 2), denoting the macroscopic energy density corresponding to $E_{\delta}$ by $\varphi^{\delta}$,

$$
\lim _{k \rightarrow \infty} \frac{1}{\nu k^{2}} \inf _{y \in \hat{\mathcal{N}}_{k, c_{0}-\delta_{2}}^{l_{2}, l_{3}}} E_{\delta_{1}}(y)=\int_{\mathcal{S}_{1}} \varphi_{c_{0}-\delta_{2}}^{\delta_{1}}(\nabla u, \mathbf{b}) \leqq \int_{\mathcal{S}_{1}} \varphi_{c_{0}-\delta_{2}}(\nabla u, \mathbf{b})
$$

for $l_{2}, l_{3} \rightarrow 0, k l_{3} \rightarrow \infty$, and hence also

$$
\limsup _{k \rightarrow \infty} \frac{1}{\nu k^{2}} \inf _{y \in \hat{\mathcal{N}}_{k, c_{0}}^{l_{2}, l_{3}}(u, \mathbf{b})} E(y) \leqq \int_{\mathcal{S}_{1}} \varphi_{c_{0}-\delta_{2}}(\nabla u, \mathbf{b}) .
$$

Now this holds for all $\delta_{2}$, therefore

$$
\limsup _{k \rightarrow \infty} \frac{1}{\nu k^{2}} \inf _{y \in \hat{\mathcal{N}}_{k, c_{0}}^{l_{2}, l_{3}}(u, \mathbf{b})} E(y) \leqq \int_{\mathcal{S}_{1}} \varphi_{c_{0}}(\nabla u, \mathbf{b})
$$


by dominated convergence, provided $\varphi_{c_{0}-\delta} \rightarrow \varphi_{c_{0}}$ boundedly on $\left\{\left|B^{i}\right| \leqq c_{0}-\theta\right\}$ as $\delta \rightarrow 0$. To see this, note first that on this set we may replace $\varphi$ by $\varphi^{\delta_{0}}$ for $\delta_{0}>0$ small enough only depending on $\theta$ (see the proof of Proposition 3 ). Now an easy consequence of Lemma 9 is that $\left|\varphi_{k, c_{0}-\delta}^{\delta_{0}}-\varphi_{k, c_{0}}^{\delta_{0}}\right| \leqq C \delta^{1 / 5}$. It remains to note that $y^{(k)} \in \hat{\mathcal{N}}_{k, c_{0}}^{l_{2}, l_{3}}(u, \mathbf{b})$ for all $k$ implies $y^{(k)} \rightarrow(u, \mathbf{b})$.

3. Now drop the assumption $\left|B^{i}\right|<c_{0}$, but still suppose that $\left|M^{\theta}\right|=0$ for some fixed $\theta>0$. Define approximations $\mathbf{b}_{\eta} \stackrel{\eta \rightarrow 0}{\longrightarrow} \mathbf{b}$ in $L^{\infty}$ by

$$
B_{\eta}^{i}= \begin{cases}B^{i} & \text { if }\left|B^{i}\right| \leqq c_{0}-\eta \\ \left(c_{0}-\eta\right) \frac{B^{i}}{\left|B^{i}\right|} & \text { if }\left|B^{i}\right|>c_{0}-\eta\end{cases}
$$

By continuity and boundedness of $\varphi$ on $\left(M^{\theta}\right)^{c}$,

$$
\lim _{\eta \rightarrow 0} \int_{\mathcal{S}_{1}} \varphi\left(\nabla u, \mathbf{b}_{\eta}\right)=\int_{\mathcal{S}_{1}} \varphi(\nabla u, \mathbf{b}) .
$$

Now choose an appropriate diagonal sequence $y^{(k)} \rightarrow(u, \mathbf{b})$ with

$$
\limsup _{k \rightarrow \infty} \frac{1}{v k^{2}} E\left(y^{(k)}\right) \leqq \int_{\mathcal{S}_{1}} \varphi(\nabla u, \mathbf{b}) .
$$

4. For general $(u, \mathbf{b})$ we may suppose that $\left|M^{0}\right|=0$ (for $\left|M^{0}\right|>0$ the upper bound is trivial). For given $\mathbf{b} \in L^{\infty}\left(\mathcal{S}_{1} ;\left(\mathbb{R}^{3}\right)^{\nu-1}\right)$ we define $\mathbf{b}_{\theta}$ by $\mathbf{b}_{\theta}(x)=\mathbf{b}(x)$ if $x \notin M^{\theta}, \mathbf{b}_{\theta} \equiv \mathbf{0}$ else. By the previous results, $|\varphi(\nabla u(x), \mathbf{0})| \leqq C$. Since $\mathbf{b}_{\theta} \stackrel{*}{\rightarrow} \mathbf{b}$, we again find $y^{(k)} \rightarrow(u, \mathbf{b})$ such that

$$
\limsup _{k \rightarrow \infty} \frac{1}{\nu k^{2}} E\left(y^{(k)}\right) \leqq \limsup _{\theta \rightarrow 0} \int_{\mathcal{S}_{1}} \varphi\left(\nabla u, \mathbf{b}_{\theta}\right) \leqq \int_{\mathcal{S}_{1}} \varphi(\nabla u, \mathbf{b})
$$

by Proposition 3 .

\subsection{Extensions and variants}

In the last paragraph of this section we discuss some extensions of the theory and possible changes of our set-up.

\subsubsection{Basic extensions}

General Bravais lattices and domains More generally, we could deal with Bravais-lattices

$$
\mathcal{L}=\left\{x \in \mathbb{R}^{3}: x=\sum_{i=1}^{3} \mu_{i} e_{i}, \mu_{i} \in \mathbb{Z}\right\},
$$

where $\left(e_{1}, e_{2}, e_{3}\right)$ are linearly independent in $\mathbb{R}^{3}$ and $\mathcal{S}_{k}:=\left\{x_{1} e_{1}+x_{2} e_{2}: x_{1}, x_{2} \in\right.$ $[0, k]\}$ for $k \in \mathbb{N}$. Then our reference configuration will be $\mathcal{L} \cap\left(\mathcal{S}_{k} \times[0, h] e_{3}\right)$ where $h:=(v-1)$, and $\Delta^{i} y\left(x_{p}\right)=y\left(x_{p}+i e_{3}\right)-y\left(x_{p}\right), x_{p} \in \mathcal{S}_{k}$. This amounts to a simple coordinate change in the physical space $\mathbb{R}^{3}$.

Covering $\mathcal{S}$ with mesoscopic squares up to a negligible error at the boundary, it is not hard to see that the convergence scheme in fact applies to bounded Lipschitz domains $\mathcal{S} \subset \mathbb{R}^{2}$ (where $\varphi$ is given as in Theorem 2). 
Alternative definition of convergence In our definition of convergence $y^{(k)} \rightarrow$ $(u, \mathbf{b})$, it is not possible to consider the limiting case of very restricted relaxation, that is $c_{0} \rightarrow 0$, unless all $b^{i}$ are zero. Instead of asking $\|\tilde{y}-u\|$ in Definition 2 to be less than $c_{0} / k$ one could demand that

$$
\|\tilde{y}-v\| \leqq c_{0} / k
$$

where $v$ is as in (10) corresponding to $u$, $\mathbf{b}$ with $b^{0}$ set to zero. (Condition (4) is not needed for this definition of convergence.) The results are analogous.

Different types of atoms The theory developed so far may be generalized to films consisting of more than one species of atoms. Then $E$ does not only depend on the positions $y_{i}$ of the atoms but also on their type, labeled by, say, $t(i) \in\{1, \ldots, s\}$,

$$
E=E\left(y_{1}, t(1), \ldots, y_{N}, t(N)\right) .
$$

Note that in our derivation we only made use of translational invariance of $E$. The theory still applies if the atoms of different type are arranged periodically on the lattice with some fixed (microscopic) period, that is, there exist $p_{1}, p_{2} \in \mathbb{N}$ such that for all $x$ the atoms at $\left(x_{1}, x_{2}, x_{3}\right),\left(x_{1}+p_{1}, x_{2}, x_{3}\right)$ and $\left(x_{1}, x_{2}+p_{2}, x_{3}\right)$ are of the same type.

3.6.2. Distinguishable particle systems Similarly, the convergence scheme also applies to certain systems with distinguishable particles. We will state a general result for systems with finite range interaction. The basic assumption is that only atoms that are close in the reference configuration are supposed to interact. This violates Assumption 2 since the energy is not a function of atomic positions in the deformed configuration any more. It rather also depends on the reference configuration, that is, the atoms are distinguishable. It will be clear, however, that the convergence scheme described so far still applies.

Let $a>0$. To each $x_{i} \in \mathcal{L}_{k}$ we assign a neighborhood

$$
U_{x_{i}}=\left\{x_{j} \in \mathcal{L}:\left|x_{j}-x_{i}\right| \leqq a\right\}=\left\{x_{1}^{i}, \ldots, x_{r_{a}}^{i}\right\},
$$

where the enumeration of elements of $U_{x_{i}}$ shall be such that $x_{1}^{i}=x_{i}$ and, if $\left(x_{i_{1}}\right)_{3}=\left(x_{i_{2}}\right)_{3}$, then $x_{j}^{i_{1}}-x_{i_{1}}=x_{j}^{i_{2}}-x_{i_{2}}$ for $j=1, \ldots, r_{a}$.

Our goal is to study energy functions of the form

$$
E_{\mathrm{fr}}(y)=\sum_{x_{i} \in \mathcal{L} \cap\left([a, k-a]^{2} \times[0, h]\right)} f_{x_{i}}\left(y\left(x_{2}^{i}\right)-y\left(x_{1}^{i}\right), \ldots, y\left(x_{r_{a}}^{i}\right)-y\left(x_{1}^{i}\right)\right)+\mathcal{O}(k),
$$

where $f_{x_{i}}: \mathbb{R}^{3\left(r_{a}-1\right)} \rightarrow \mathbb{R}$ are given functions representing the energy of the interactions between the $i$ th atom at its position $y\left(x_{i}\right)=y\left(x_{1}^{i}\right)$ and its neighboring atoms in their positions $y\left(x_{2}^{i}\right), \ldots, y\left(x_{r_{a}}^{i}\right)$. (The term $\mathcal{O}(k)$ is introduced to compensate for boundary effects since $U_{x_{i}}$ is not contained in $S_{k} \times[0, h]$ for $x_{i}$ in a boundary layer of constant width $a$.) 
More precisely, since we also have to consider energies of subsets of our atomic lattice, suppose the $f_{x_{i}}$ are functions on $\left(\mathbb{R}^{3} \cup\{\alpha\}\right)^{r_{a}-1}$ with $\alpha \notin \mathbb{R}^{3}$ and $\operatorname{dist}(\alpha, x):=1$ for all $x \in \mathbb{R}^{3}$. For a subset $\mathcal{K}$ of $\mathcal{L}_{k}$ we define

$$
E_{\mathrm{fr}}(y(\mathcal{K}))=\sum_{x_{i} \in \mathcal{K}} f_{x_{i}}\left(y\left(x_{2}^{i}\right)-y\left(x_{1}^{i}\right), \ldots, y\left(x_{r_{a}}^{i}\right)-y\left(x_{1}^{i}\right)\right)
$$

with $y\left(x_{j}^{i}\right)-y\left(x_{1}^{i}\right)$ replaced by $\alpha$ whenever $x_{j}^{i} \notin \mathcal{K}$.

We do not assume $f_{x_{i}}$ to satisfy any symmetry conditions. However, as noted earlier, we do need some periodicity, so we suppose there exist fixed $p_{1}, p_{2} \in \mathbb{N}$ such that

$$
\begin{array}{r}
f_{\left(x_{1}+p_{1}, x_{2}, x_{3}\right)}=f_{x}=f_{\left(x_{1}, x_{2}+p_{2}, x_{3}\right)} \\
\text { for } x=\left(x_{1}, x_{2}, x_{3}\right) \in\left(\mathbb{Z}_{+}\right)^{2} \times\{0, \ldots, v-1\} .
\end{array}
$$

Proposition 4. Suppose $E_{\mathrm{fr}}$ is defined as in (50) and (51) holds. Assume that the $f_{x_{i}}$ are locally Lipschitz. Then the limit $\varphi_{\mathrm{fr}}$ of Theorem 2 exists, and we have

$$
\lim _{k \rightarrow \infty} \frac{1}{\nu k^{2}} \inf _{y \in \mathcal{W}_{k}^{l}(u, \mathbf{b})} E_{\mathrm{fr}}(y)=\int_{\mathcal{S}_{1}} \varphi_{\mathrm{fr}}(\nabla u(x), \mathbf{b}(x)) \mathrm{d} x
$$

as $l \rightarrow 0$ and $k l \rightarrow \infty$.

(Adopting the notion of $\delta$-criticality suitably (compare Definition 4), also unbounded pair-interaction parts can be treated analogously to Theorem 4.)

Sketch of Proof. First note that by (51) there are only finitely many different functions $f_{x}$. Due to Lemma 1, a bound on the distance of two atoms in the reference configuration implies a bound on their distance in the deformed state. So by a cut-off argument we may suppose that the functions $f_{x_{i}}$ are uniformly bounded and have common Lipschitz constants. But each atom occurs in at most $r_{a}$ summands of (50). This proves the desired Lipschitz property of $E$. As noted earlier, the remaining part of Assumption 2 can be weakened to requiring that the periodicity assumption (51) is satisfied.

As for Assumption 1, to estimate

$$
\mid E\left(y\left(\mathcal{K}_{1} \cup \mathcal{K}_{2}\right)-E\left(y\left(\mathcal{K}_{1}\right)\right)-E\left(y\left(\mathcal{K}_{2}\right)\right) \mid\right.
$$

note that, if $x_{i} \in \mathcal{K}_{1}$ is such that $U_{x_{i}} \cap\left(\mathcal{K}_{1} \cup \mathcal{K}_{2}\right) \neq U_{x_{i}} \cap \mathcal{K}_{1}$, then there exists $x^{\prime} \in U_{x_{i}} \cap \mathcal{K}_{2}$, that is, by Lemma $1,\left|y(x)-y\left(x^{\prime}\right)\right| \leqq C$, a constant, analogously for $\mathcal{K}_{1}, \mathcal{K}_{2}$ interchanged. On the other hand, due to the uniform boundedness of the $f_{x_{i}} \mathrm{~s}$, the error term can be estimated by a constant $\left(C^{\prime}\right.$, say) times the number $(N$, say) of such $x_{i}$ in $\mathcal{K}_{1} \cup \mathcal{K}_{2}$. Now if $\psi=2 C^{\prime} \chi_{\{x:|x| \leqq C\}}$, then indeed

$$
\mid E\left(y\left(\mathcal{K}_{1} \cup \mathcal{K}_{2}\right)-E\left(\mathcal{K}_{1}\right)-E\left(\mathcal{K}_{2}\right) \mid \leqq C^{\prime} N \leqq \sum_{x \in \mathcal{K}_{1}, x^{\prime} \in \mathcal{K}_{2}} \psi\left(\left|y(x)-y\left(x^{\prime}\right)\right|\right)\right.
$$

Remark. Dealing only with interactions whose range is bounded in the reference configuration, there is no need for a minimal strain hypothesis on $u$, that is, for these interactions we might set $c_{1}=0$ in (3). 


\section{Examples/applications}

In this section, we will investigate some examples of atomic interactions and explore under what circumstances these models fit into the theory developed in the last section. The first three models will satisfy Assumptions 1 and 2 even in the more restrictive sense of Assumption 3. For the last one this will be obviously false. Throughout this discussion we will assume that $u \in W^{1, \infty}\left(\mathcal{S}_{1} ; \mathbb{R}^{3}\right), b^{i} \in$ $L^{\infty}\left(S_{1} ; \mathbb{R}^{3}\right)$ are admissible. Applying the chain rule $\nabla f \circ g(x)=f^{\prime}(g(x)) \nabla g(x)$ almost everywhere for Lipschitz functions $f: \mathbb{R} \rightarrow \mathbb{R}, g: \mathbb{R}^{n} \rightarrow \mathbb{R}$, as usual, the right-hand side is interpreted as zero whenever $\nabla g=0$ regardless of $f^{\prime}(g(x))$ being well-defined or not.

\subsection{Pair potentials}

As a first example we consider pair potentials, that is, energy functions of the form

$$
E_{\mathrm{pp}}(y)=\frac{1}{2} \sum_{i \neq j} W\left(\left|y_{i}-y_{j}\right|\right),
$$

where $W:[0, \infty) \rightarrow \mathbb{R}$.

Proposition 5. Suppose $E_{\mathrm{pp}}$ is defined as in (52). Assume that $W:[0, \infty) \rightarrow \mathbb{R}$ is Lipschitz. If there exist $M>0$ and $q>3$ such that for almost every $r \geqq 0$

$$
|W(r)| \leqq M r^{-q} \text { and }\left|W^{\prime}(r)\right| \leqq M r^{-q+1},
$$

then $E_{\mathrm{pp}}$ is admissible.

Proof. We need only check that $E_{\mathrm{pp}}$ satisfies Assumptions 1 and 2. Clearly, $E_{\mathrm{pp}}$ only depends on atomic positions, is frame indifferent, and satisfies Assumption 1 with $\psi(r)=|W(r)|$. Furthermore, $W$ Lipschitz (with Lipschitz constant $M^{\prime}$, say) implies that $E$ is Lipschitz, and we have almost everywhere

$$
\begin{aligned}
\left|\frac{\partial E}{\partial y_{l}}(y)\right| & =\left|\frac{1}{2} \sum_{i \neq j} W^{\prime}\left(\left|y_{i}-y_{j}\right|\right) \cdot \frac{y_{i}-y_{j}}{\left|y_{i}-y_{j}\right|} \cdot\left(\delta_{i l}-\delta_{j l}\right)\right| \\
& \leqq \sum_{j \neq l}\left|W^{\prime}\left(\left|y_{l}-y_{j}\right|\right)\right| .
\end{aligned}
$$

We have to find a bound on this quantity assuming $\|\tilde{y}-u\| \leqq C / k$. But then as in Lemma $1 y$ satisfies $|y(x)-y(z)| \geqq C_{1}|x-z|-C_{3}$, and we can apply the technique of splitting the sum into long-range and short-range terms as in the proof of Lemma 6. From $\left|W^{\prime}(r)\right| \leqq M^{\prime}$ and $\left|W^{\prime}(r)\right| \leqq M r^{-q+1}$ (if existing) for some $q>3$, we then deduce that the right-hand side of (53) is bounded almost everywhere (independently of $k$ and $l$ ).

An example is given by the Morse potential with interaction function

$$
W_{\mathrm{M}}(r):=W_{0}\left(e^{-2 a\left(r-r_{0}\right)}-2 e^{-a\left(r-r_{0}\right)}\right)
$$

for positive parameters $W_{0}, a$ and $r_{0}$ (cf. [29]). 
Having proven this proposition independently of Theorem 4, also pair potentials with $W$ as in (16) are covered by our convergence scheme, for example, the
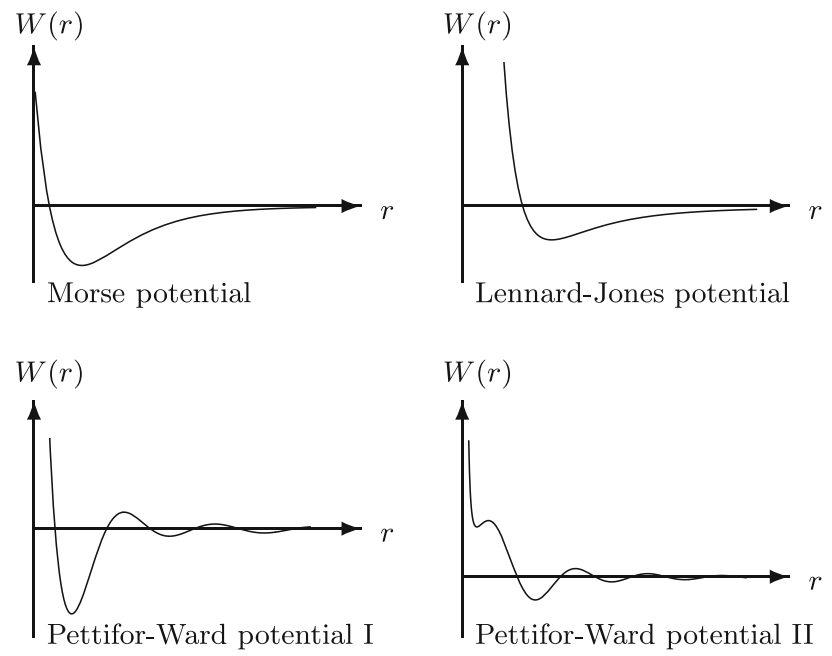

Lennard-Jones potential given by

$$
W_{\mathrm{LJ}}(r)=W_{0} \cdot\left(\left(\frac{\sigma}{r}\right)^{12}-\left(\frac{\sigma}{r}\right)^{6}\right),
$$

$W_{0}>0$ and $\sigma$ constants (compare [29]), and the Pettifor-Ward pair potentials (compare [33]) given by

$$
W_{\mathrm{PW}}(r)=\frac{W_{0}}{r} \sum_{n=1}^{3} a_{n} \cos \left(k_{n} r+\alpha_{n}\right) e^{-\kappa_{n} r},
$$

$W_{0}>0, a_{n}, k_{n}, \alpha_{n}, \kappa_{n}$ constants such that $\sum_{n} a_{n} \cos \left(\alpha_{n}\right)>0$.

\subsection{Pair functionals}

More generally, in this paragraph we will discuss pair functionals as examples of the embedded atom method. These models have the advantage of also covering some environmental dependence of the bond strength between the nuclei at positions $\left\{y_{i}\right\}$ (compare [29]). We let

$$
E_{\mathrm{pf}}(y)=\frac{1}{2} \sum_{i \neq j} W\left(\left|y_{i}-y_{j}\right|\right)+\sum_{i} F\left(\rho_{i}\right),
$$

where $W:[0, \infty) \rightarrow \mathbb{R}$ as above, $F:[0, \infty) \rightarrow \mathbb{R}$, and $\rho_{i}$ is given by

$$
\rho_{i}=\sum_{j \neq i} f\left(\left|y_{i}-y_{j}\right|\right)
$$

$f:[0, \infty) \rightarrow[0, \infty)$ 
The interpretation of such an energy function is the following (compare [29]). As always, $\left\{y_{i}\right\}$ denotes the positions of the nuclei of some material. These nuclei are supposed to be embedded in some electron gas consisting of the valence electrons of the atoms of that material. Now suppose that the total energy associated with $y$ can be split into two parts: one that describes the interaction of the various nuclei, leading to the first summand in (54), and the sum of the energy it costs to embed a single nucleus into an electron gas of some density $\rho$. Denoting this energy

$$
E_{\text {embedding }}=F(\rho) \text {, }
$$

where $\rho$ denotes the electron density at the point the nucleus is embedded, and assuming that the electron density at $y_{i}$ depends on the positions of the other nuclei through

$$
\rho_{i}=\sum_{j \neq i} f\left(\left|y_{i}-y_{j}\right|\right)
$$

this embedding energy of a single nucleus at $y_{i}$ is indeed $F\left(\rho_{i}\right)$.

We aim at exhibiting conditions on $W, F$ and $f$ such that $E_{\mathrm{pf}}$ satisfies Assumptions 1 and 2. First note that since

$$
E_{\mathrm{pf}}(y)=\frac{1}{2} \sum_{i \neq j} W\left(\left|y_{i}-y_{j}\right|\right)+\sum_{i} F\left(\sum_{j \neq i} f\left(\left|y_{i}-y_{j}\right|\right)\right),
$$

$E_{\text {pf }}$ only depends on atomic positions and, depending in fact only on the interatomic distances, is frame indifferent.

Lemma 21. Suppose $E_{\mathrm{pf}}$ is defined as in (54) and $W$ is as in Proposition 5 (respectively Theorem 4$)$. Assume $F:[0, \infty) \rightarrow(-\infty, 0]$ is convex and Lipschitz, $f:[0, \infty) \rightarrow[0, \infty)$ is Lipschitz and, for almost every $r \geqq 0$,

$$
|F \circ f(r)| \leqq M r^{-q}, \quad\left|f^{\prime}(r)\right| \leqq M r^{-q+1} .
$$

Then $E_{\mathrm{pf}}$ is admissible (respectively Theorem 4 applies).

Note that — as is plausible — by the decay hypothesis and assumptions on $F$, necessarily $f(r) \rightarrow 0$ as $r \rightarrow \infty$ (if $F$ is not trivial). In the following proposition we will see that $F$ need not be Lipschitz. While the decay assumption on $f^{\prime}$ is in the spirit of the previous result, $|F \circ f(r)| \leqq M r^{-q}$ poses quite severe decay conditions on $f$, if we take, for example, $F(a) \sim \sqrt{a}$. This will be remedied in Proposition 6.

Proof. First note that $F \leqq 0$ being convex implies that $-F$ is subadditive. By Proposition 5 it remains to verify Assumptions 1 and 2 for the embedding term $E_{\mathrm{emb}}(y)=\sum_{i} F\left(\rho_{i}\right)$. So let $\mathcal{M}$ and $\mathcal{N}$ be disjoint sets of atoms. Setting

$$
\rho_{v}^{\mathcal{K}}=\sum_{\substack{w \in \mathcal{K} \\ w \neq v}} f(|v-w|)
$$


we find

$$
\begin{aligned}
& \left|E_{\mathrm{emb}}(\mathcal{M} \cup \mathcal{N})-E_{\mathrm{emb}}(\mathcal{M})-E_{\mathrm{emb}}(\mathcal{N})\right| \\
& \quad=\left|\sum_{v \in \mathcal{M} \cup \mathcal{N}} F\left(\rho_{v}^{\mathcal{M} \cup \mathcal{N}}\right)-\sum_{v \in \mathcal{M}} F\left(\rho_{v}^{\mathcal{M}}\right)-\sum_{v \in \mathcal{N}} F\left(\rho_{v}^{\mathcal{N}}\right)\right| \\
& \quad=\left|\sum_{v \in \mathcal{M}}\left(F\left(\rho_{v}^{\mathcal{M} \cup \mathcal{N}}\right)-F\left(\rho_{v}^{\mathcal{M}}\right)\right)+\sum_{v \in \mathcal{N}}\left(F\left(\rho_{v}^{\mathcal{M} \cup \mathcal{N}}\right)-F\left(\rho_{v}^{\mathcal{N}}\right)\right)\right| .
\end{aligned}
$$

Consider the first sum: $f \geqq 0$ implies that

$$
\rho_{v}^{\mathcal{M} \cup \mathcal{N}}=\sum_{\substack{w \in \mathcal{M} \cup \mathcal{N} \\ w \neq v}} f(|v-w|) \geqq \sum_{\substack{w \in \mathcal{M} \\ w \neq v}} f(|v-w|)=\rho_{v}^{\mathcal{M}}
$$

So since $F$ is decreasing (because it is convex and non-positive), we have

$$
\begin{aligned}
& \left|\sum_{v \in \mathcal{M}}\left(F\left(\rho_{v}^{\mathcal{M} \cup \mathcal{N}}\right)-F\left(\rho_{v}^{\mathcal{M}}\right)\right)\right|=\sum_{v \in \mathcal{M}}\left(-F\left(\rho_{v}^{\mathcal{M} \cup \mathcal{N}}\right)+F\left(\rho_{v}^{\mathcal{M}}\right)\right) \\
& \left.\quad \leqq \sum_{v \in \mathcal{M}}\left(\left[-F\left(\sum_{\substack{w \in \mathcal{M} \\
w \neq v}} f(|v-w|)\right)+\sum_{w \in \mathcal{N}}-F(f(|v-w|))\right]+F\left(\rho_{v}^{\mathcal{M}}\right)\right)\right] \\
& \quad=\sum_{v \in \mathcal{M}} \sum_{w \in \mathcal{N}}-F(f(|v-w|))
\end{aligned}
$$

by subadditivity of $-F$. Treating the term $\left|\sum_{v \in \mathcal{N}}\left(F\left(\rho_{v}^{\mathcal{M} \cup \mathcal{N}}\right)-F\left(\rho_{v}^{\mathcal{N}}\right)\right)\right|$ analogously and summing up, we have shown that

$$
\left|E_{\mathrm{emb}}(\mathcal{M} \cup \mathcal{N})-E_{\mathrm{emb}}(\mathcal{M})-E_{\mathrm{emb}}(\mathcal{N})\right| \leqq \sum_{\substack{v \in \mathcal{M} \\ w \in \mathcal{N}^{\prime}}}-2 F \circ f(|v-w|)
$$

so we may choose $\psi(r)=-2 F \circ f(r)$. Note that since $f$ is bounded, $F \circ f$ is bounded too. Clearly the decay hypothesis on $\psi(r)$ as $r \rightarrow \infty$ is satisfied. This concludes the first part of the proof.

For the remaining part we again only need to consider the embedding term of the energy. (The first one is dealt with as in the proof of Proposition 5.) $F$ is 
Lipschitz, say $\left\|F^{\prime}\right\|_{\infty} \leqq M^{\prime}$. So almost everywhere

$$
\begin{aligned}
& \left|\frac{\partial}{\partial y_{l}} \sum_{i} F\left(\sum_{j \neq i} f\left(\left|y_{i}-y_{j}\right|\right)\right)\right| \\
& =\left|\sum_{i}\left(F^{\prime}\left(\sum_{j \neq i} f\left(\left|y_{i}-y_{j}\right|\right)\right) \cdot \sum_{j \neq i} f^{\prime}\left(\left|y_{i}-y_{j}\right|\right) \cdot \frac{y_{i}-y_{j}}{\left|y_{i}-y_{j}\right|} \cdot\left(\delta_{i l}-\delta_{j l}\right)\right)\right| \\
& \leqq M^{\prime}\left|\sum_{i \neq j} f^{\prime}\left(\left|y_{i}-y_{j}\right|\right) \cdot \frac{y_{i}-y_{j}}{\left|y_{i}-y_{j}\right|} \cdot\left(\delta_{i l}-\delta_{j l}\right)\right| \\
& \leqq 2 M^{\prime} \sum_{j \neq l}\left|f^{\prime}\left(\left|y_{l}-y_{j}\right|\right)\right|
\end{aligned}
$$

Just as before, for $\tilde{y}$ in a $C / k$-neighborhood of $u$, the decay and boundedness hypotheses on $f^{\prime}$ allow us to split this sum into long-range and short-range terms. We thus find a bound on this quantity independent of $k$ and $l$.

Proposition 6. Suppose $W$ is as in Proposition 5 (respectively Theorem 4). Assume now $F:[0, \infty) \rightarrow(-\infty, 0]$ is convex, $f:[0, \infty) \rightarrow(0, \infty)$ is Lipschitz, and, for almost every $r \geqq 0$,

$$
|f(r)| \leqq M r^{-q}, \quad\left|f^{\prime}(r)\right| \leqq M r^{-q+1} .
$$

Then Theorems 1, 2, and 3 (respectively 4) apply to $E_{\mathrm{pf}}$ as given in (54) and (55).

Remark. Before we prove this proposition we would like to comment on the plausibility of the various assumptions. $F$ is non-positive since placing a positively charged particle into an electron cloud yields energy. The non-negativity of $f$ is clear since $f$ is supposed to be a density. Strict positivity is plausible since perfect screening is not to be expected. The convexity condition on $F$ can be understood as reflecting the fact that, due to screening, adding more electrons, that is, raising the electron density, results in smaller and smaller effects. This seems to match experimental data (compare [29], p. 171). A qualitatively reasonable scaling would be given by $F(a) \sim-\sqrt{a}$ as, for example, in the Finnis-Sinclair model where $F(a) \propto-\sqrt{a}$ (compare [29]). The remaining are decay assumptions on $f$ similar to those for $W$.

Proof. Let $y$ be some deformation satisfying $\|\tilde{y}-u\| \leqq C / k$. Then for each $y_{i}=y\left(x_{i}\right)$ there is $y_{j}=y\left(x_{j}\right)$ with $j \neq i$ and $\left|y_{i}-y_{j}\right| \leqq 2 C+c_{2}\left(\operatorname{choose} x_{j}\right.$ to be a neighbor of $x_{i}$ ). So $\sum_{j \neq i} f\left(\left|y_{i}-y_{j}\right|\right.$ ) (i fixed) is bounded from below by some $\delta>0$. Defining $\hat{F}$ suitably by

$$
\hat{F}(\rho)=\left\{\begin{array}{cl}
0 & \text { for } \rho=0, \\
\text { linear } & \text { for } 0 \leqq \rho \leqq \delta, \\
F(\rho) & \text { for } \rho \geqq \delta,
\end{array}\right.
$$


$\hat{F}$ is convex and Lipschitz. Furthermore, $|\hat{F} \circ f(r)| \leqq \frac{|F(\delta)|}{\delta}|f(r)| \leqq C M r^{-q}$. So the corresponding energy $\hat{E}_{\mathrm{pf}}(y)$ is admissible. Since for all $y$ with $\|\tilde{y}-u\| \leqq c_{0} / k$

$$
E_{\mathrm{pf}}(y)=\hat{E}_{\mathrm{pf}}(y),
$$

Theorems 1, 2, and 3 also apply to $E$.

Remark. $E_{\mathrm{pf}}$ is not admissible in the usual sense since, for example, for two atoms $y_{1}, y_{2}$

$$
E_{\mathrm{pf}}\left(y_{1}, y_{2}\right)=W\left(\left|y_{1}-y_{2}\right|\right)+2 F\left(f\left(\left|y_{1}-y_{2}\right|\right)\right),
$$

and $F \circ f(r)$ is in general not $\mathcal{O}\left(r^{-q}\right)$ for some $q>3$.

\subsection{Angular forces}

In this paragraph we consider energy functions that may also depend on the angles between atomic bonds. For a physical motivation of such models we refer to [29]. Mathematically this leads to the consideration of potentials depending on triplets of atomic positions:

$$
E_{\mathrm{af}}(y)=\frac{1}{2} \sum_{i \neq j} W\left(\left|y_{i}-y_{j}\right|\right)+\frac{1}{6} \sum_{\substack{i, j, k \\ i \neq j \neq k \neq i}} \hat{W}\left(y_{i}, y_{j}, y_{k}\right),
$$

where $W:[0, \infty) \rightarrow \mathbb{R}$, and $\hat{W}$ is given by

$$
\begin{aligned}
\hat{W}\left(y_{i}, y_{j}, y_{k}\right)= & h\left(\left|y_{i}-y_{j}\right|,\left|y_{j}-y_{k}\right|, \theta_{i j k}\right)+h\left(\left|y_{j}-y_{k}\right|,\left|y_{k}-y_{i}\right|, \theta_{j k i}\right) \\
& +h\left(\left|y_{k}-y_{i}\right|,\left|y_{i}-y_{j}\right|, \theta_{k i j}\right),
\end{aligned}
$$

$\theta_{i j k}$ denoting the angle between $y_{i}-y_{j}$ and $y_{k}-y_{j}$, and

$$
h:\left\{\begin{array}{l}
{[0, \infty) \times[0, \infty) \times \mathbb{R} \rightarrow \mathbb{R},} \\
\left(r_{1}, r_{2}, \theta\right) \mapsto h\left(r_{1}, r_{2}, \theta\right),
\end{array}\right.
$$

is $2 \pi$-periodic and symmetric in the last variable.

Again we are seeking conditions on $W$ and $\hat{W}$ (respectively $h$ ) such that $E_{\text {af }}$ satisfies Assumptions 1 and 2. As before, it is easy to see that $E_{\mathrm{af}}(y)$ depending only on inter-atomic distances and angles is determined by atomic positions and is frame indifferent.

Proposition 7. Suppose $E_{\mathrm{af}}$ is defined as in (57). Assume that $W$ is as in Proposition 5 (respectively Theorem 4 ) and $h$ is Lipschitz. Furthermore, there are bounded functions $\chi_{1}, \chi_{2}, \alpha_{1}, \alpha_{2}:[0, \infty) \rightarrow[0, \infty)$ with

$$
\chi_{\mu}(r) \leqq M r^{-q}, \quad \alpha_{\mu}(r) \leqq M r^{-q+1}, \quad \mu=1,2
$$

such that

$$
\left|h\left(r_{1}, r_{2}, \theta\right)\right| \leqq \chi_{1}\left(r_{1}\right) \chi_{2}\left(r_{2}\right)
$$

and (almost everywhere) 


$$
\left|\frac{\partial h}{\partial r_{\mu}}\left(r_{1}, r_{2}, \theta\right)\right| \leqq \alpha_{1}\left(r_{1}\right) \alpha_{2}\left(r_{2}\right), \quad \mu=1,2,
$$

and

$$
\left|\frac{\partial h}{\partial \theta}\left(r_{1}, r_{2}, \theta\right)\right| \leqq \alpha_{1}\left(r_{1}\right) \alpha_{2}\left(r_{2}\right) \min \left\{r_{1}, r_{2}\right\} .
$$

Then $E_{\mathrm{af}}$ is admissible (respectively Theorem 4 applicable).

Remark. Note that it is plausible to require that $\partial h / \partial \theta$ vanish as $r_{1} \rightarrow 0$ or $r_{2} \rightarrow 0$ since $\hat{W}\left(y_{i}, y_{j}, y_{k}\right)$ should depend continuously on $y_{i}, y_{j}, y_{k}$, but the angle $\theta_{i j k}$ does not when the triangle becomes degenerate.

The proof is tedious but not very hard. Splitting into long- and short-range terms, all sums occurring in the error terms can be bounded appropriately. $\psi$ can be chosen as $\psi(r)=|W(r)|+C \max \left\{\chi_{1}(r), \chi_{2}(r)\right\}$.

Example. If $h$ splits into

$$
h\left(r_{1}, r_{2}, \theta\right)=f_{1}\left(r_{1}\right) f_{2}\left(r_{2}\right) g(\theta),
$$

as, for example, for Stillinger-Weber-type energies (compare [29]). Then $h$ satisfies the conditions of Proposition 7 if $f_{\mu}, f_{\mu}^{\prime}$ are bounded, $\left|f_{\mu}\right| \leqq M r^{-q},\left|f_{\mu}^{\prime}\right| \leqq$ $M r^{-q+1}$ for $\mu=1,2, f_{1}\left(r_{1}\right) f_{2}\left(r_{2}\right) \leqq \min \left\{r_{1}, r_{2}\right\}$ and $g$ and $g^{\prime}$ are bounded. This is satisfied, for example, for the angular term

$$
g(\theta)=(\cos (\theta)+1 / 3)^{2}
$$

discussed in [29].

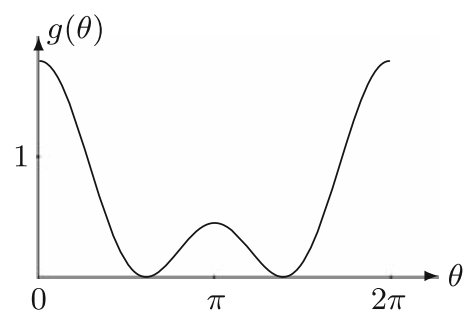

\subsection{A simple example}

Even for fairly elementary microscopic energies as, for example, given by pair potentials, not much is known about their ground state deformations. (Some onedimensional results in this direction can be found in [5], a recent two-dimensional result for certain pair potentials is proven in [34].) We conclude this section calculating $\varphi$ explicitly for a simple nearest neighbor model. Although it lacks some physical requirements (for example shear resistance), it captures some realistic features as, for example, quadratic energy growth near the reference configuration (a natural state) for pure tensions. The model consisting of two different types of bonds, the energy minimizer will not be a simple crystal. A pointwise limit would overestimate the macroscopic energy. 
Suppose the atoms of our reference configuration interact only with nearest neighbors and the interaction potential is harmonic, that is, given by springs of strength $d_{1}$ and $d_{2}$ with equilibrium at distance 1 .

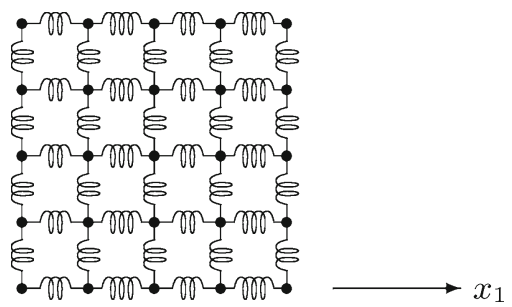

We assume that bonds in the reference configuration parallel to the $x_{2}$ - or $x_{3}$-axes have $d_{1}=1$, while bonds parallel to the $x_{1}$ axis have alternating $d_{1}=1$ and $d_{2}=2$ as in the previous picture. So the energy is given by

$$
E_{\mathrm{nn}}(y)=\frac{1}{2} \sum_{\left|x_{i}-x_{j}\right|=1} d_{i j}\left(\left|y_{i}-y_{j}\right|-1\right)^{2},
$$

$d_{i j}=1$ or 2 as described above.

Proposition 8. $E_{\mathrm{nn}}$ is admissible in the sense of Proposition 4. In particular, the limit $\varphi_{\mathrm{nn}}$ of Theorem 2 exists for $E_{\mathrm{nn}}$ and

$$
E_{\mathrm{nn}}(u, \mathbf{b})=\int_{\mathcal{S}_{1}} \varphi_{\mathrm{nn}}(\nabla u(x), \mathbf{b}(x)) \mathrm{d} x .
$$

Furthermore (set $\left.b^{0}=0\right)$, if $c_{0}$ is not too small,

$$
\begin{aligned}
\varphi_{\mathrm{nn}}(A, \mathbf{b})= & \frac{4}{3}(\max \{0,|a \cdot 1|-1\})^{2}+(\max \{0,|a \cdot 2|-1\})^{2} \\
& +\sum_{i=1}^{\nu-1}\left(\max \left\{0,\left|b^{i}-b^{i-1}\right|-1\right\}\right)^{2}
\end{aligned}
$$

where $a_{. j}$ denotes the jth column of $A$.

This is clearly a special case of (50) with $a=1$ and periodicity $p_{1}=2, p_{2}=1$. So we only have to prove the representation formula for $\varphi_{\mathrm{nn}}$.

Sketch of Proof. The main observation in the elementary but tedious proof is that the energy decouples into energies of one dimensional atomic chains

$$
i \mapsto y\left(i, x_{2}, x_{3}\right), \quad \text { resp. } i \mapsto y\left(x_{1}, i, x_{3}\right), \quad i=0, \ldots, k,
$$

with $k+1$ atoms $\left(\left(x_{2}, x_{3}\right)\right.$ respectively $\left(x_{1}, x_{3}\right)$ fixed), and $v-1$ chains with $(k+1)^{2}+1$ atoms whose difference of successive atoms (labeled by $0 \leqq x_{1}, x_{2} \leqq k$ ) is given by $y\left(x_{1}, x_{2}, i\right)-y\left(x_{1}, x_{2}, i-1\right), i$ fixed: 


$$
\begin{aligned}
E(y)= & \sum_{\substack{0 \leqq x_{2} \leqq k \\
0 \leqq x_{3} \leqq \nu-1}} \sum_{0 \leqq x_{1} \leqq k-1} d\left(x_{1}\right)\left(\left|y\left(x_{1}+1, x_{2}, x_{3}\right)-y\left(x_{1}, x_{2}, x_{3}\right)\right|-1\right)^{2} \\
& +\sum_{\substack{0 \leqq x_{1} \leqq k \\
0 \leqq x_{3} \leqq \nu-1}} \sum_{0 \leqq x_{2} \leqq k-1}\left(\left|y\left(x_{1}, x_{2}+1, x_{3}\right)-y\left(x_{1}, x_{2}, x_{3}\right)\right|-1\right)^{2} \\
& +\sum_{0 \leqq x_{3} \leqq \nu-2} \sum_{0 \leqq x_{1}, x_{2} \leqq k}\left(\left|y\left(x_{1}, x_{2}, x_{3}+1\right)-y\left(x_{1}, x_{2}, x_{3}\right)\right|-1\right)^{2},
\end{aligned}
$$

where $d\left(x_{1}\right)=d_{1}=1$ if $x_{1}$ is even, $d\left(x_{1}\right)=d_{2}=2$ if $x_{1}$ is odd. Now the energy can be bounded from below by minimizing the energy of these chains separately subject to boundary conditions $\tilde{y}=v$ on $\partial S_{1} \times[0, h]$ respectively $f \Delta^{i} \tilde{y}=b^{i}$. Allowing for negligible error terms, these configurations can be patched together to yield the desired result.

Acknowledgments. The present results are part of my $\mathrm{Ph} . \mathrm{D}$. thesis [32]. I am grateful to my Ph.D. supervisor Prof. S. Müller for his guidance, support and helpful advice. Also I would like to thank Prof. G. FrIESECKE for stimulating discussions during a two weeks visit at Warwick University. This work was supported by the German science foundation (DFG) under project FOR522.

\section{References}

1. Alicandro, R., Cicalese, M.: A general integral representation result for continuum limits of discrete energies with superlinear growth. SIAM J. Math. Anal. 36, 1-37 (2004)

2. Alicandro, R., Braides, A., Cicalese, M.: Continuum limits of discrete thin films with superlinear growth densities. Preprint 2005. http://cvgmt.sns.it/papers/alibracic05/

3. Antman, S.S.: Nonlinear Problems of Elasticity. Springer, Berlin, 1995

4. Anzellotti, G., Baldo, S., Percivale, D.: Dimension reduction in variational problems, asymptotic development in $\Gamma$-convergence and thin structures in elasticity. Asymptotic Anal. 9, 61-100 (1994)

5. Blanc, X., LeBris, C.: Periodicity of the infinite-volume ground state of a onedimensional quantum model. Nonlinear Anal. Theory Methods Appl. 48A, 791-803 (2002)

6. Blanc, X., LeBris, C., Lions, P.-L.: Convergence de modèles moléculaires vers des modèles de mécanique des milieux continus. C. R. Acad. Sci. Paris 332, 949-956 (2001)

7. Blanc, X., LeBris, C., Lions, P.-L.: From molecular models to continuum mechanics. Arch. Rational Mech. Anal. 164, 341-381 (2002)

8. Braides, A.: Nonlocal variational limits of discrete systems. Commun. Contemp. Math. 2, 285-297 (2000)

9. Braides, A., Gelli, M.S.: Limits of discrete systems with long-range interactions. J. Convex Anal. 9, 363-399 (2002)

10. Braides, A., Gelli, M.S.: Continuum limits of discrete systems without convexity hypotheses. Math. Mech. Solids 7, 41-66 (2002)

11. Ciarlet, P.G.: Mathematical Elasticity, vol. I: Three-dimensional Elasticity. NorthHolland, Amsterdam, 1988

12. Ciarlet, P.G.: Mathematical Elasticity, vol. II: Theory of Plates. North-Holland, Amsterdam, 1997

13. Dacorogna, B.: Direct Methods in the Calculus of Variations. Springer, Berlin, 1989

14. Dal Maso, G.: An Introduction to $\Gamma$-convergence. Birkhäuser, Boston, 1993 
15. Euler, L.: Methodus Inveniendi Lineas Curvas, Additamentum I: De Curvis Elasticis (1744). In: Opera Omnia Ser. Prima vol. XXIV, pp. 231-297. Orell Füssli, Bern 1952

16. Evans, L.C., GARIEPY, R.F.: Measure Theory and Fine Properties of Functions. CRC Press, Boca Raton, 1992

17. Friesecke, G., James, R.D.: A scheme for the passage from atomic to continuum theory for thin films, nanotubes and nanorods. J. Mech. Phys. Solids 48, 1519-1540 (2000)

18. Friesecke, G., James, R.D., MülLER, S.: Rigorous derivation of nonlinear plate theory and geometric rigidity. C. R. Acad. Sci. Paris 334, 173-178 (2002)

19. Friesecke, G., James, R.D., Müller, S.: A theorem on geometric rigidity and the derivation of nonlinear plate theory from three-dimensional elasticity. Commun. Pure Appl. Math. 55, 1461-1506 (2002)

20. Friesecke, G., James, R.D., Müller, S.: A hierarchy of plate models derived from nonlinear elasticity by Gamma-convergence. Arch. Rational Mech. Anal. 180, 183-236 (2006)

21. Friesecke, G., James, R.D., Mora, M.G., Müller, S.: Derivation of nonlinear bending theory for shells from three-dimensional nonlinear elasticity by Gamma-convergence. C. R. Acad. Sci. Paris 336, 697-702 (2003)

22. Friesecke, G., James, R.D., Mora, M.G., Müller, S.: Derivation of the nonlinear bending-torsion theory for inextensible rods by $\Gamma$-convergence. Calc. Var. Partial Differ. Equ. 18, 287-305 (2003)

23. vON KÁRMán, T.: Festigkeitsprobleme im Maschinenbau. In: Encyclopädie der Mathematischen Wissenschaften, vol. IV/4, pp. 311-385, Leipzig, 1910

24. KirchinofF, G.: Über das Gleichgewicht und die Bewegung einer elastischen Scheibe. J. Reine Angew. Math. 40, 51-88 (1850)

25. Le Dret, H., Raoult, A.: La modèle membrane non linéaire comme limite variationnelle de l'élasticité non linéaire tridimensionnelle. C. R. Acad. Sci. Paris 317, 221-226 (1993)

26. Le Dret, H., Raoult, A.: The nonlinear membrane model as a variational limit of three-dimensional elaticity. J. Math. Pures Appl. 74, 549-578 (1995)

27. Le Dret, H., Raoult, A.: The membrane shell model in nonlinear elaticity: a variational asymptotic derivation. J. Nonlinear Sci. 6, 59-84 (1996)

28. Love, A.E.H.: A Treatise on the Mathematical Theory of Elasticity. Cambridge University Press, Cambridge, 1927

29. Phillips, R.: Crystals, Defects and Microstructures. Cambridge University Press, Cambridge, 2001

30. Schmidt, B.: Qualitative properties of a continuum theory for thin films. Ann. Inst. Henri Poincaré, Anal. Non Linéaire 25, 43-75 (2008)

31. Schmidt, B.: A derivation of continuum nonlinear plate theory from atomistic models. SIAM Multiscale Model. Simul. 5, 664-694 (2006)

32. Sснмidt, B.: Effective Theories for Thin Elastic Films. Ph.D. thesis, University of Leipzig, 2006

33. Sutton, A.P.: Electronic Structure of Materials. Oxford University Press, Oxford, 1994

34. Theil, F.: A proof of crystallization in two dimensions. Commun. Math. Phys. 262, 209-236 (2005) 
Max-Planck-Institute for Mathematics in the Sciences, Inselstr. 22, 04103 Leipzig, Germany. e-mail: bschmidt@mis.mpg.de e-mail: bschmidt@aero.caltech.edu

and

Present Address:

Zentrum Mathematik,

Technische Universität München,

Boltzmannstr. 3,

85747 Garching bei München, Germany.

e-mail: schmidt@ma.tum.de

(Received December 1, 2005 / Accepted February 19, 2007)

Published online August 9, 2008 - (C) Springer-Verlag (2008) 